Aus der Abteilung Hals-Nasen-Ohrenheilkunde

(Prof. Dr. med. Ch. Matthias)

im Zentrum Augenheilkunde und Hals-Nasen-Ohrenheilkunde

der Medizinischen Fakultät der Universität Göttingen

\title{
Electroretinography and exploration of visual and auditory function in mutant mice with synaptic defects
}

\author{
INAUGURAL - DISSERTATION \\ zur Erlangung des Doktorgrades \\ der Medizinischen Fakultät \\ der Georg-August-Universität zu Göttingen
}

vorgelegt von

Gabriele Cornelia Maria Bauer

aus

Marburg a. d. Lahn

Göttingen 2012 
Dekan:

I. Berichterstatter: Prof. Dr. med. T. Moser

II. Berichterstatter: Prof. Dr. med. T. Gollisch

Tag der mündlichen Prüfung: 20. November 2012 


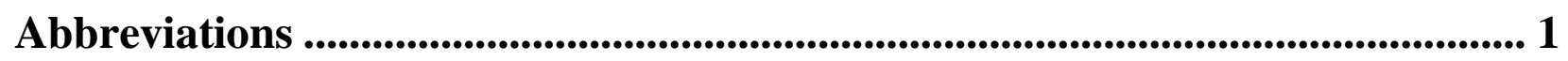

1 Introduction ....................................................................................................................... 4

1.1 Intention of this work ...........................................................................................

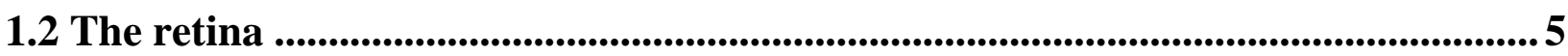

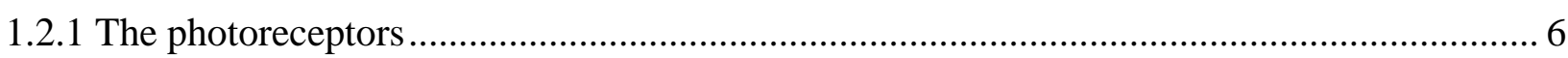

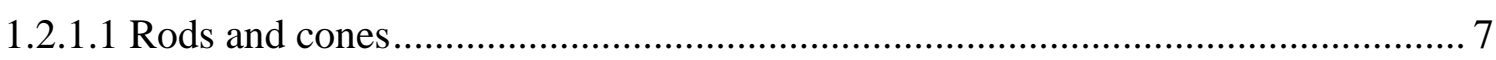

1.2.1.2 The photoreceptor current .............................................................................. 7

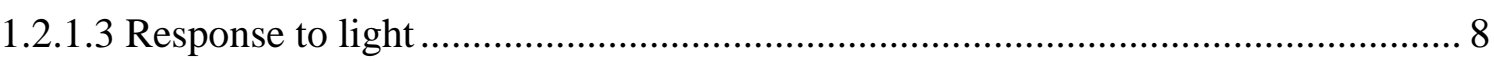

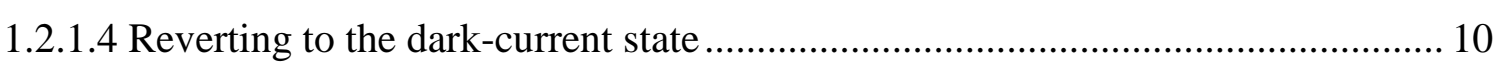

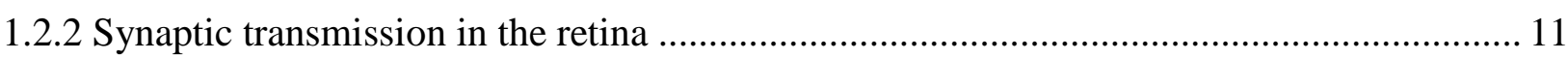

1.2.2.1 Excitatory transmission - Glutamatergic ribbon synapses ................................... 11

1.2.2.2 Inhibitory transmission - GABA and Glycine receptors...................................... 13

1.2.2.3 Synaptic transmission in the OPL .................................................................. 15

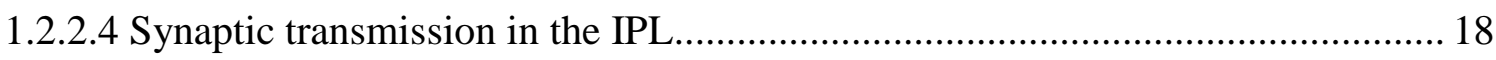

1.3 The electroretinogram (ERG) .........................................................................19

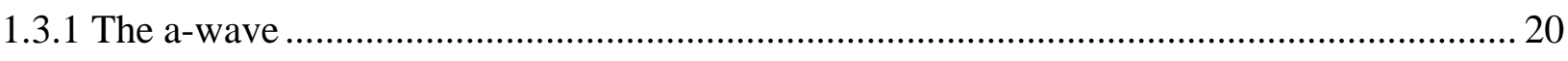

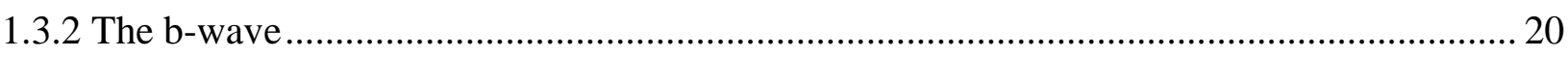

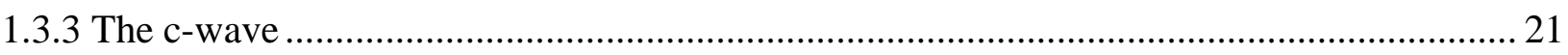

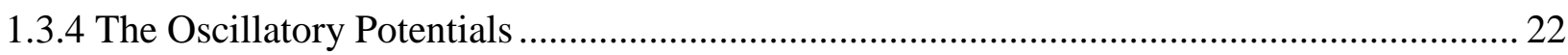

1.4 Investigated Proteins ................................................................................................23

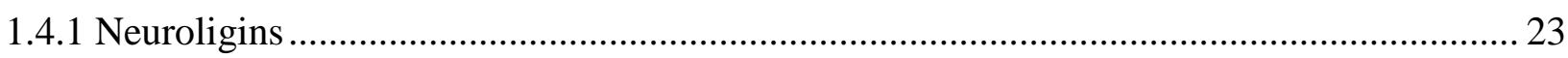

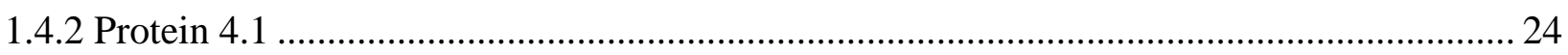

2 Materials and Methods................................................................................. 26

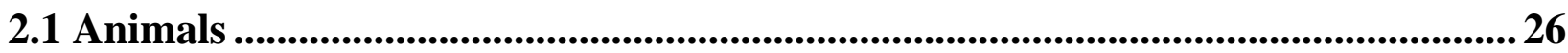

2.2 Electroretinograms - ERG …....................................................................................................... 27

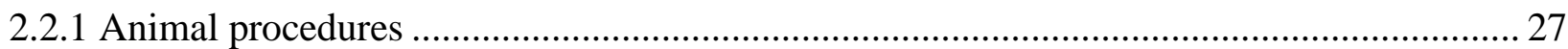

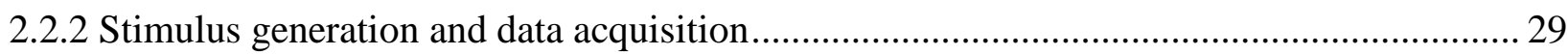

2.3 Data Analysis........................................................................................................................33

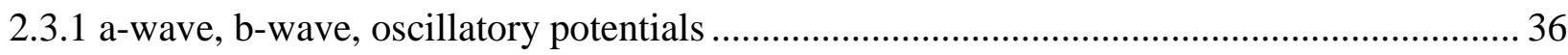

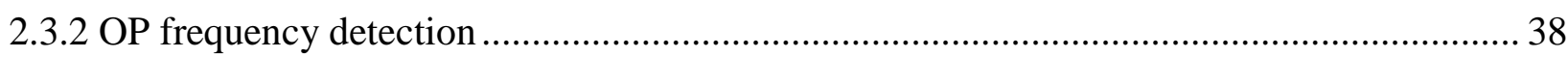


3.1 Electroretinograms ..................................................................................................................4 41

3.2 Auditory brainstem response ..................................................................................................... 41

3.3 Neuroligin 2 ............................................................................................................................................ 42

3.3.1 Scotopic ERG measurements - Examples..................................................................... 43

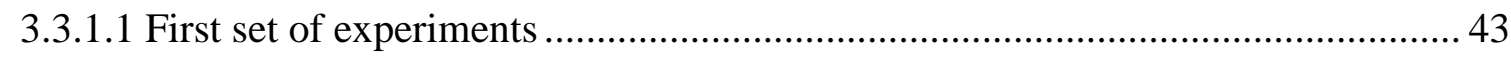

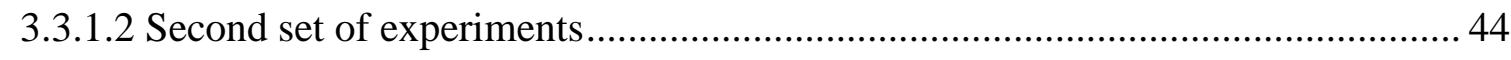

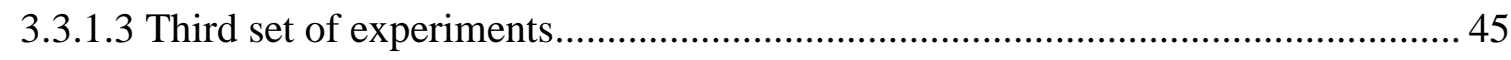

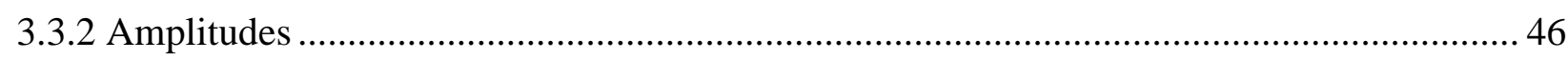

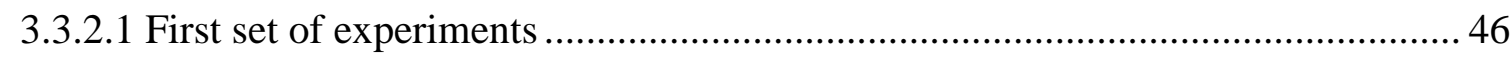

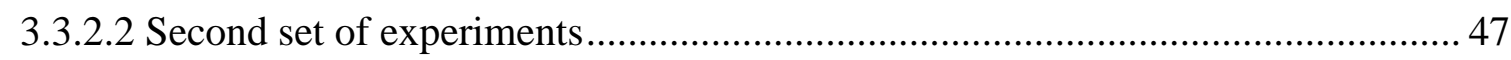

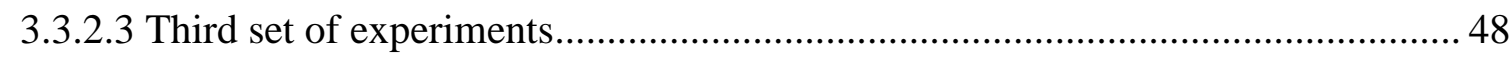

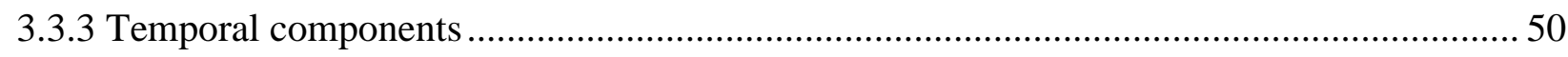

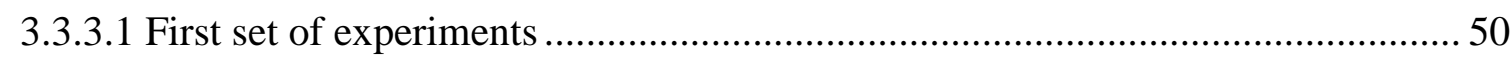

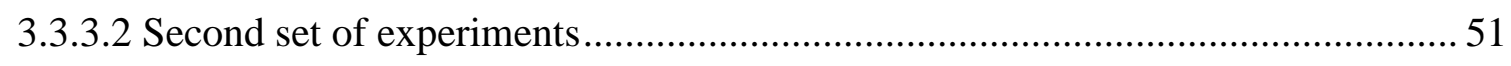

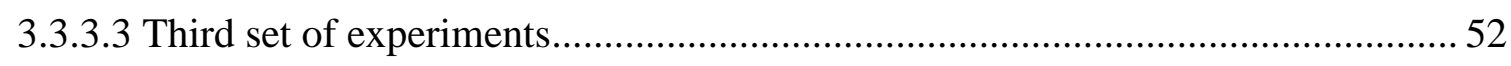

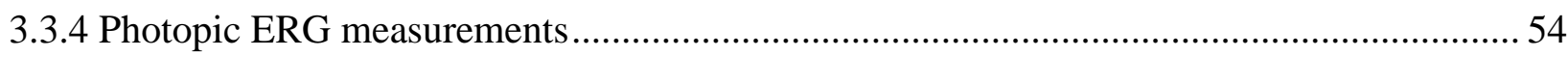

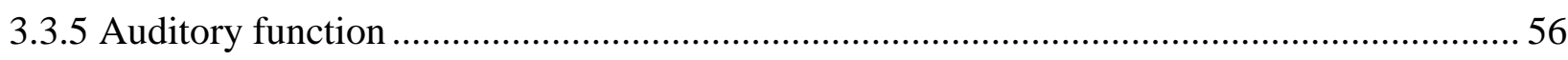

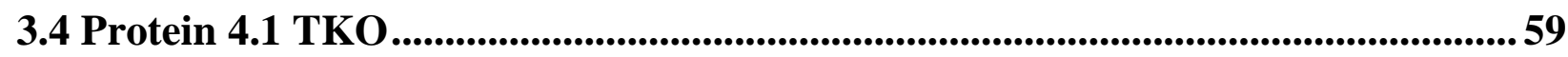

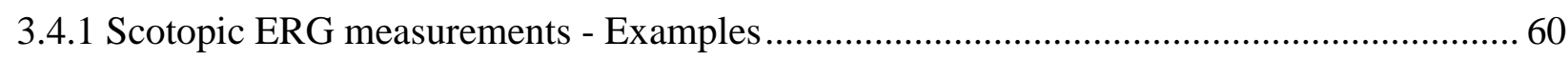

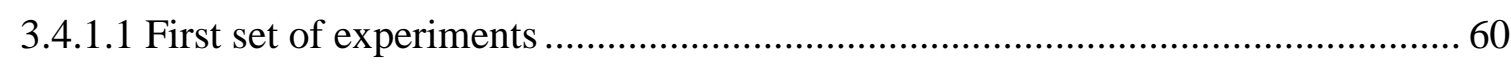

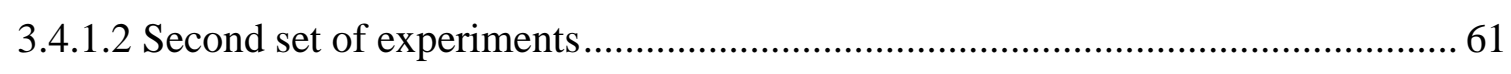

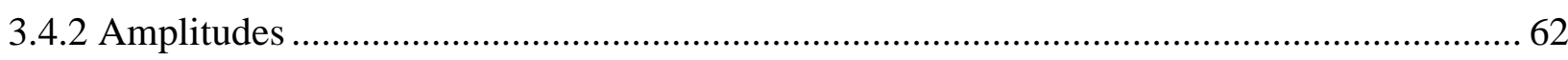

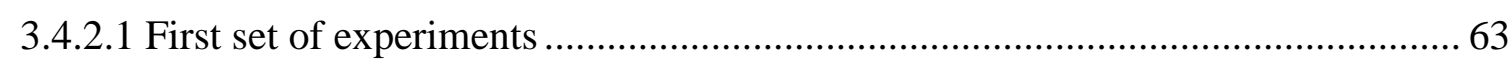

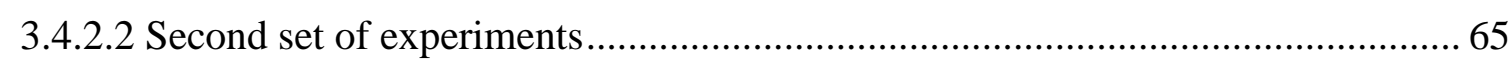

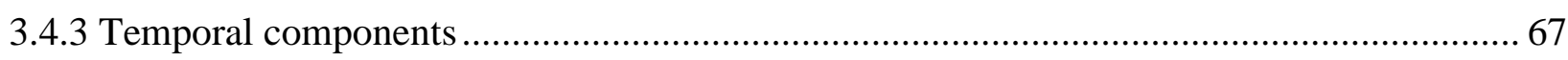

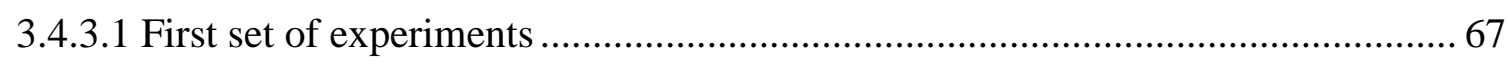

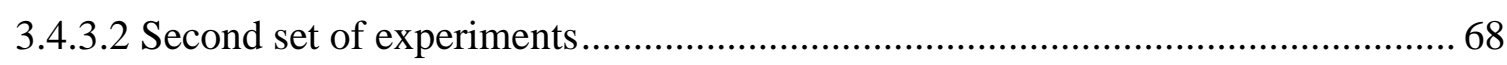

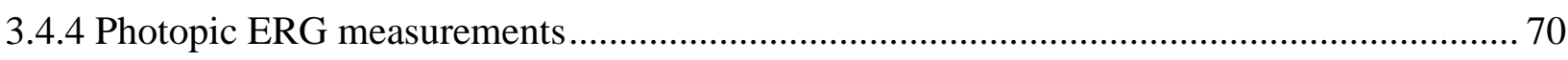

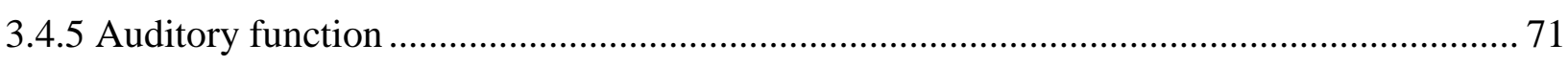

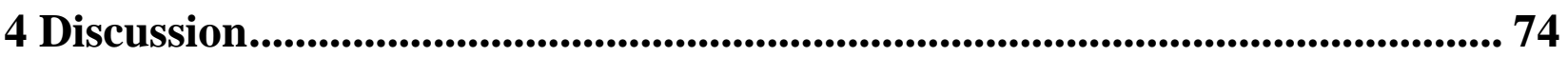

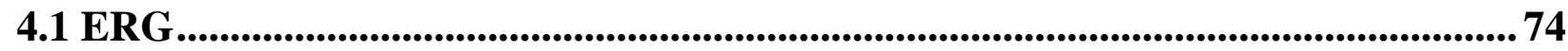

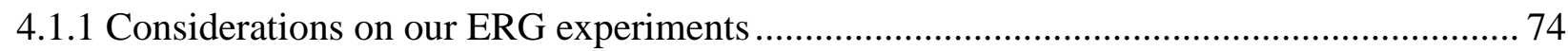

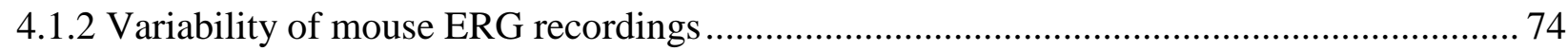




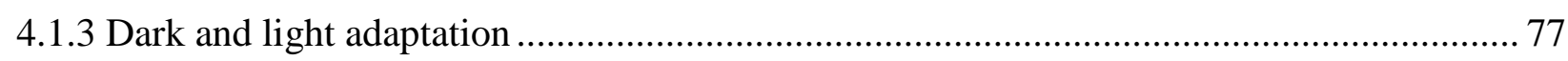

4.1.4 Adaptation and exhaustion of the retina - Inter Stimulus Interval .................................. 78

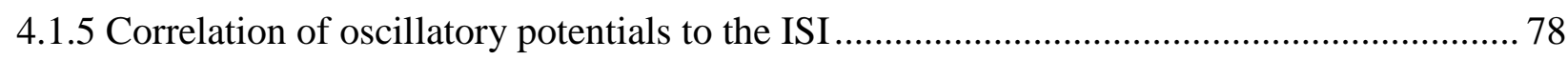

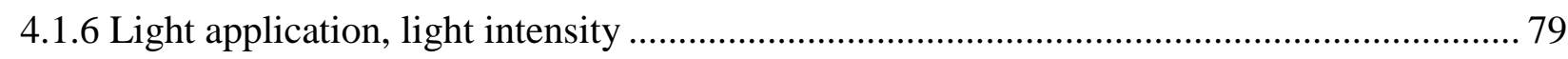

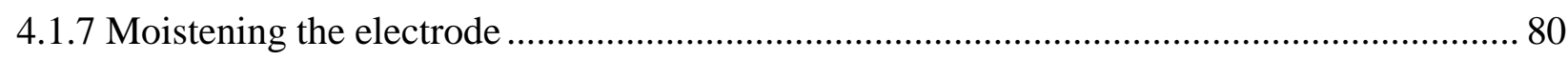

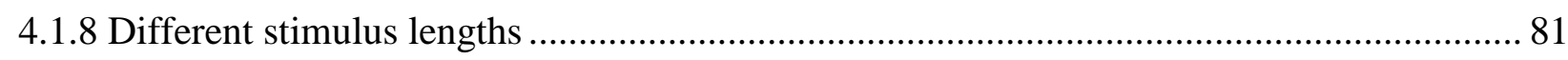

4.2 Neuroligin 2 ............................................................................................................. 82

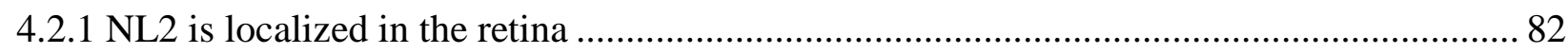

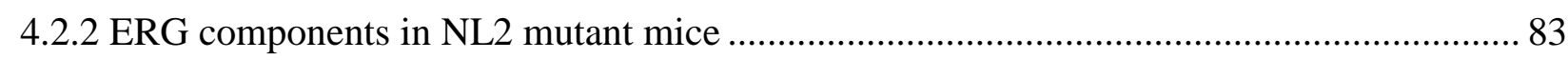

4.2.2.1 Possible reasons for reduced ERG OP amplitudes in NL2 mutant retinae .......... 83

4.2.2.2 Possible reasons for reduced ERG b-wave amplitudes in NL2 mutant retinae ... 84

4.2.2.3 Possible reasons for reduced ERG a-wave amplitudes in NL2 mutant retinae.... 86

4.2.3 Recovery kinetics - the key to non-reproducible results? ............................................... 86

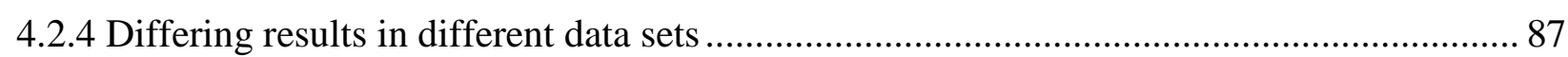

4.3 Protein 4.1 .........................................................................................................................8 89

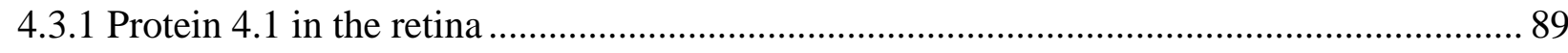

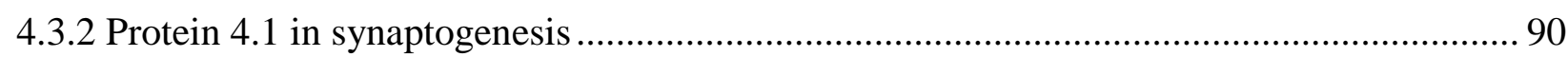

4.3.3 Protein 4.1 modulates glutamatergic retinal signaling ................................................. 91

5 Summary ................................................................................................................... 94

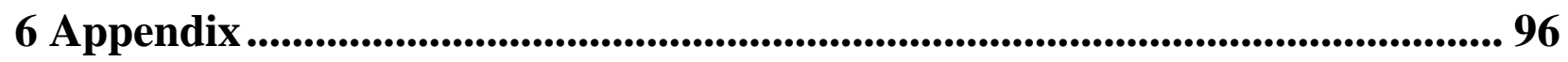

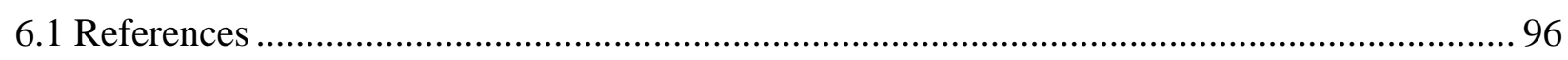

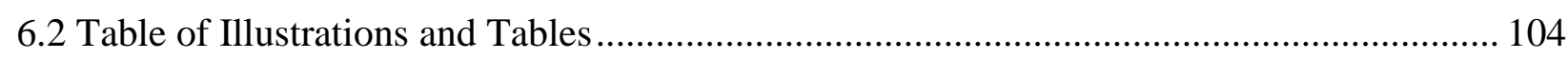




\section{Abbreviations}

$+/+$

$-/-$

ABR

AC

$\mathrm{Ag} / \mathrm{AgCl}$

$\mathrm{AgCl}$

AMPA

APB

ASSR

$\mathrm{Ba}^{+}$

$\mathrm{BC}(\mathrm{s})$

$\mathrm{Ca}^{2+}$

(c)ATP/ADP/AMP

cd

cds

(c)GTP/GDP/GMP

$\mathrm{Cl}^{-}$

$\mathrm{CNG}$

CNS

DB

DNA

DTL

EAEP

ECG

EEG

e.g.

ERG
Wildtype

Knockout

Auditory Brainstem Response

Amacrine Cell

Silver/Silver Chloride

Silver Chloride

$\alpha$-amino-3-hydroxy-5-methyl-4-isoxazolepropionic acid

2-amino-4-phosphonobutyric acid

Auditory Steady State Response

Barium

Bipolar Cell(s)

Calcium

(Cyclic) Adenosinetriphosphate/-diphosphate/-monophosphate

Candela

Candela Second

(Cyclic) Guanosinetriphosphate/-diphosphate/-monophoshate

Chloride

Cyclic Nucleotide Gated

Central Nervous System

Dezibel

Deoxyribonucleic Acid

Dawson Trick Litzkow

Early Acoustic Evoked Potentials

Electrocardiogram

Electroencephalogram

exempli gratia (for example)

Electroretinogram 


\begin{tabular}{|c|c|}
\hline et al. & et alii (and others) \\
\hline$f$ & Frequency \\
\hline $\mathrm{g}$ & Grams \\
\hline GABA & Gamma-Amino-Butyric-Acid \\
\hline GC & Ganglion Cell \\
\hline GCAP & Guanylyl Cyclase Activating Protein \\
\hline GCL & Ganglion Cell Layer \\
\hline GCy & Guanylyl Cyclase \\
\hline GluR & Glutamate Receptor \\
\hline GlyR & Glycine Receptor \\
\hline GlyT & Glycine Transporter \\
\hline $\mathrm{HC}$ & Horizontal Cell \\
\hline $\mathrm{Hz}$ & Hertz \\
\hline iGluR & Ionotropic Glutamate Receptor \\
\hline ILM & Inner Limiting Membrane \\
\hline INL & Inner Nuclear Layer \\
\hline IPL & Inner Plexiform Layer \\
\hline IS & Inner Segment \\
\hline ISI & Inter Stimulus Interval \\
\hline $\mathrm{K}^{+}$ & Potassium \\
\hline $\mathrm{KO}$ & Knock-Out \\
\hline LED & Light Emitting Diode \\
\hline M.D. & Medical Doctor \\
\hline $\mathrm{mg}$ & Milligrams \\
\hline mGluR & Metabotropic Glutamate Receptor \\
\hline $\mathrm{ms}$ & Milliseconds \\
\hline$\mu \mathrm{V}$ & Microvolts \\
\hline $\mathrm{n}$ & Number \\
\hline
\end{tabular}


$\mathrm{Na}^{+}$

NL2

NMDA

NT

OLM

ONL

OPL

$\mathrm{OP}(\mathrm{s})$

OS

PDA

PDE

PR

PSD

RK

(m)RNA

RPE

$\mathrm{Sec}$

SPL

TKO

VEP

W

WT
Sodium

Neuroligin 2

$\mathrm{N}$-methyl-D-aspartate

Neurotransmitter

Outer Limiting Membrane

Outer Nuclear Layer

Outer Plexiform Layer

Oscillatory Potential(s)

Outer Segment

cis-2,3-piperidine-dicarboxylic acid

Phosphodiesterase

Photoreceptor

Post-Synaptic Density

Rhodopsin Kinase

(Messenger) Ribonucleic Acid

Retinal Pigment Epithelium

Second(s)

Sound Pressure Level

Triple Knockout

Visually Evoked Potentials

Watts

Wildtype 


\section{Introduction}

\subsection{Intention of this work}

Objective testing of sensory function by sensory evoked potentials requires accessibility of the system without damaging the important structures. Transmission of information in the nervous system is mediated by electrical signals that can be recorded with extracellular electrodes. This way, we are able to take recordings of the retinal activity from the corneal surface. The ERG provides information about the activity of many retinal cell types. It is a compound potential representing the activity of all cell types responsive to light or involved in the process of retinal signal processing. Neurophysiological testing of vision allows assessment of the retina (electroretinogram - ERG), the optic nerve, the chiasm and retrochiasmal visual pathways (visually evoked potentials - VEP).

This project is concerned with revealing function or dysfunction of synaptic transmission throughout the retina in animals lacking specific proteins expected to be involved in synapse formation or signal transmission. The mutations investigated in this study affect proteins thought to be involved in either signaling mechanisms or synaptogenesis within the retina. The ERG was a clear option to fulfil the objective and so the paradigms of measurements and analysis were developed and further improved. This work constitutes a customized protocol for ERG recordings. Particularly interested in the function of retinal synaptic ribbons, we want to take a closer look at the function of the photoreceptor-to-bipolar cell- and the bipolar-to-ganglion cell-transmission. We are able to reveal the individual components of the ERG and draw conclusions on function or dysfunction of retinal signal transmission.

As our lab primarily focuses on auditory physiology, we also tested auditory function of the experimental animals but did not find alterations. 


\subsection{The retina}

The retina is the innermost layer of the eyeball that contains the light-sensitive photoreceptors and is the location for the first stages of visual image processing. It is organized the reverse way than expected. The photoreceptor cells lie distally at the very back of the retina and light has to pass all other retinal layers before it reaches the outermost segments of these cells, where primary light perception takes place (Kolb, 2006).

The retina consists of seven layers (Junqueira and Carneiro, 2005). The layer of photoreceptor outer segments (distal end of the retina) abuts directly on the non-neural pigment epithelium The outer nuclear layer (ONL) contains the inner segments of the photoreceptors (sensory cell/ $1^{\text {st }}$ neuron). The outer plexiform layer (OPL) follows with the synapses between axons of photoreceptors and dendrites of the bipolar cells ( $2^{\text {nd }}$ neuron) and horizontal cells. The inner nuclear layer (INL) contains the somata of bipolar cells, horizontal cells and amacrine cells. In the inner plexiform layer (IPL), axons of bipolar cells contact the dendrites of ganglion cells ( $3^{\text {rd }}$ neuron) and amacrine cells. Innermost lies the ganglion cell layer containing the somata of ganglion cells (proximal end of the retina).

Glial cells, in the retina called Muller cells, span across all seven layers. Franze et al. in 2007 proposed that they span the entire retina and serve a lightguide-like function. The Muller cells form adherent junctions with photoreceptor cell inner segments, creating the so called outer limiting membrane (OLM). The inner limiting membrane (ILM) is again formed from Muller cell terminals' membrane particles associated with other basal membrane components (Kolb, Simple Anatomy of the Retina on: http://webvision.med.utah.edu/sretina.html, downloaded Dec $6^{\text {th }}$ 2011) 


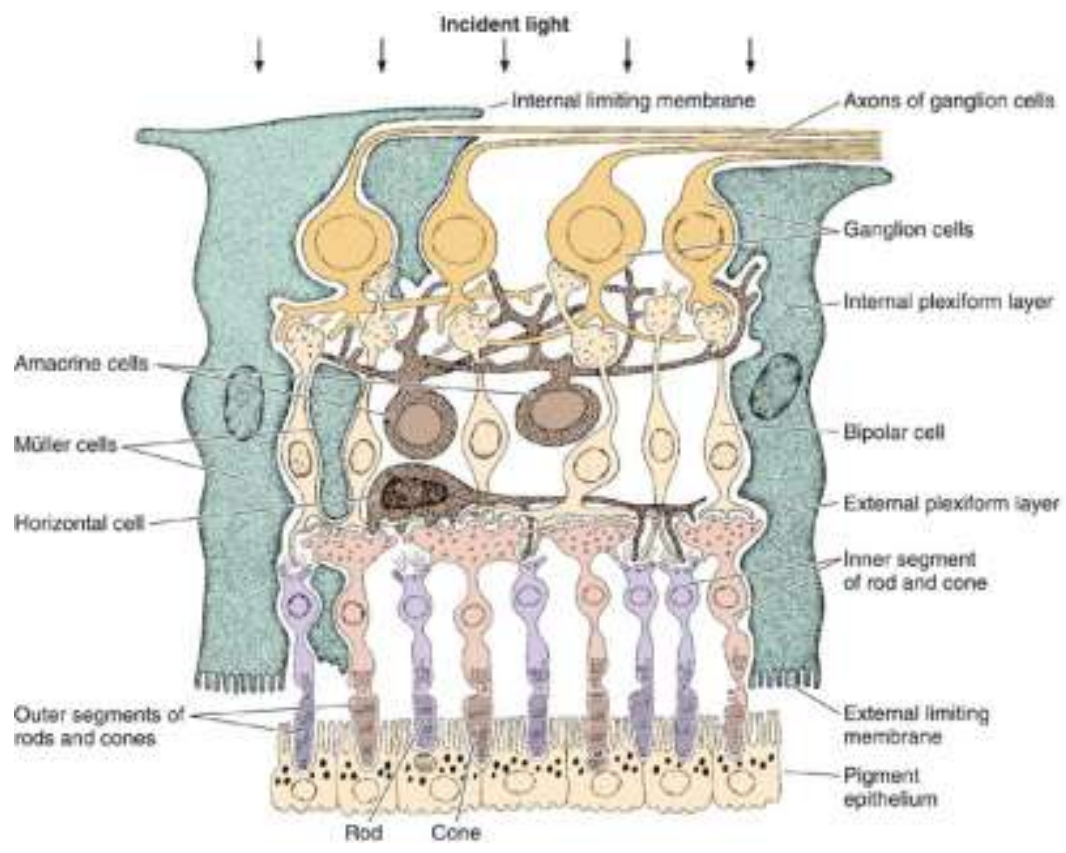

Figure 1: Microscopic schematic of the retina

Figure 1 shows a schematic of all retinal layers and cells within the layers. Innermost (proximal) lie the ganglion cells, who form the optic nerve with their axons. Towards the outermost (distal) layer follow the somata of ganglion cells, amacrine cells, bipolar cells, horizontal cells, photoreceptors and the retinal pigment epithelium. Muller glial cells span across all other layers (from: Junqueira and Carneiro, 2005, p.469f).

\subsubsection{The photoreceptors}

The photoreceptors consist of an outer segment (OS) and an inner segment (IS), which are connected via a small bridge (called a cilium, which is not a cilium in the narrower sense but a membranous cell bridge). The PR outer segments contain packed disk-shaped membranes. In rods, they form from invaginations of the plasma membrane and are separated organelles. In cones, these invaginations are still connected to the plasma membrane. The membrane disks contain a high concentration of visual pigments as well as structural and functional proteins. The inner segments contain the metabolic and synthetic machinery of the cell, including many mitochondria. The synthetic machinery of the cell is mainly dedicated to the production of vesicles filled with visual pigment (Smith, 2006; Kolb, 2006). The photoreceptor cells contain pigmentbearing membranes in their outer segments, which have to be in contact with the pigment epithelium layer. At the contact site between retina and pigment epithelium, the vitamin A derived retinal is passed from the pigment epithelium to a molecule called opsin in the photoreceptor outer segment membranes. Together, they form the light-sensitive rhodopsin molecules. The pigment epithelium phagocytoses old tissue from the photoreceptor outer segments in a diurnally designed fashion (Young, 1971). 


\subsubsection{Rods and cones}

Rod photoreceptors are very light-sensitive and function at much lower light intensities than cones. A rod cell can respond to a single photon. Rods contain more light sensitive pigment than cones, but only one type. This rod pigment is unspecifically light sensitive and rods are therefore achromatic. They represent the primary source for night- or so-called scotopic vision. Loss of rod function causes night blindness. Rods respond slowly to a light stimulus. While this makes rods more sensitive to smaller amounts of light, it also limits their ability to sense temporal changes compared to cones. The signals arising from multiple rod cells converge onto the following rod bipolar cells. Cone photoreceptors mediate day- or so-called photopic vision. Cones are much less sensitive to light than rods. In humans, usually three types of cones are present, each of which possesses a different opsin and responds to light of a certain range of wavelengths (red-, green- and blue-sensitive). In most mammals, two types of cones are present (green- and blue-sensitive). Only a few cones converge onto one following bipolar cell and they are concentrated in the fovea, the part of the retina which provides highest acuity in vision. These characteristics enable cones to perceive finer detail and rapid changes in images (Kandel et al., 2000). Rodents have a rod dominated retinal design. Their cones represent only 3-4\% of all their photoreceptors (Kolb, 2006).

\subsubsection{The photoreceptor current}

CNG sodium-channels are found in the membrane of the OS of the photoreceptors. They are cGMP-gated and mediate influx of sodium ions (and influx if $\mathrm{Ca}^{2+}$ ions). In darkness, photoreceptors contain a high concentration of cGMP opening the sodium-channels and allowing influx of sodium ions into the cell. This causes a depolarization of the photoreceptor with a membrane potential of around $-40 \mathrm{mV}$. This state of the cell-membrane in the dark is called the photoreceptor dark current. Located at the IS membrane of the photoreceptors are non-gated potassium channels mediating efflux of potassium ions. When, by closure of the $\mathrm{Na}^{+}$channels through a light stimulus, influx of $\mathrm{Na}^{+}$ions is terminated, the cell hyperpolarizes through the maintained efflux of $\mathrm{K}^{+}$ions. Through the IS outward current of potassium ions the PR membrane is hyperpolarized (up to $\sim 70 \mathrm{mV}$ ). The PR cell is able to maintain constant potassium and sodium levels via a high density of $\mathrm{Na}^{+}-\mathrm{K}^{+}$-pumps (exporting $\mathrm{Na}^{+}$, importing $\mathrm{K}^{+}$) in the IS membrane (Kandel et al., 2000). 


\subsubsection{Response to light}

The conversion of light energy into a receptor potential is called phototransduction. One detailed work on the mechanisms of phototransduction was published by Yau and Hardie in 2009. The mechanisms of the PR current have been described above. The following section with the help of fig.2 describes what happens when a light stimulus hits the PR and how this changes the membrane potential into a hyperpolarized state.

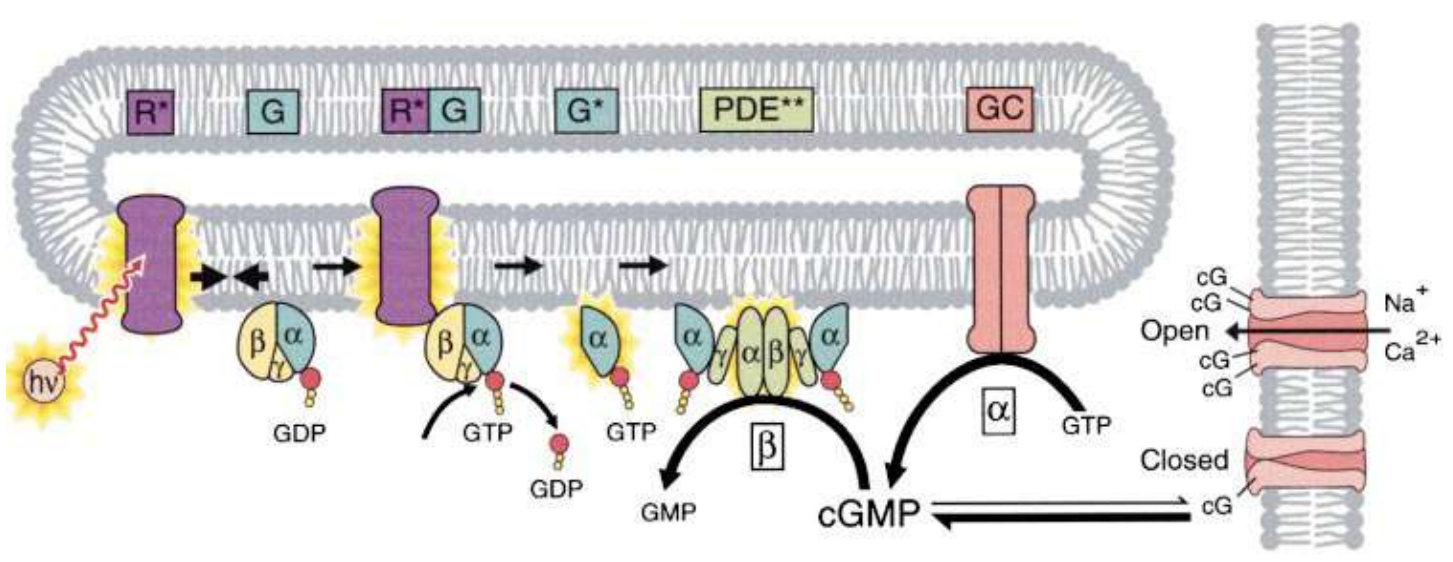

Figure 2: "Schematic of the molecular mechanisms underlying activation" (Leskov et al., 2000, p.526)

"Absorption of a photon ( $\mathrm{h} v$ ) activates a rhodopsin molecule in the disk membrane to $\mathrm{R}$ ". Each $\mathrm{R}$ * sequentially contacts numerous molecules of transducin $(G)$, catalyzing their activation to $G^{*}$ through exchange of a bound GDP for a GTP from the cytoplasm. Two $\mathrm{G}^{*} \mathrm{~s}$ can bind to each phosphodiesterase holomer, activating the PDE to PDE ${ }^{* *}$. The activated $\mathrm{PDE}^{* *}$ hydrolyzes cGMP, reducing its cytoplasmic concentration, and thereby causing closure of cGMPgated channels in the plasma membrane. The rate constant of cGMP hydrolysis by PDE $^{* *}$ is indicated by $\beta$, and the rate of cGMP synthesis by guanylyl cyclase (GCy) is indicated by $\alpha$. ." (Leskov et al., 2000, p.526)

The photoreceptive pigment rhodopsin consists of two components. The large protein opsin, which is located to the stacked membrane discs in the PR OS and the covalently bound retinal, which is passed to the PR OS from the RPE. The light absorbing portion of rhodopsin is the vitamin A derived retinal. Opsin does not absorb light. Rhodopsin is classified as a G-protein coupled receptor. In the dark, retinal is present in the 11-cis-conformation. Stimulation by light (hv) causes a structural change into the all-trans-conformation, creating an activated rhodopsin molecule $\left(\mathrm{R}^{*}\right)$. This conformational change is the only light-dependent step in vision. Opsin undergoes a conformational change and all-trans-retinal is separated from opsin. All-trans-retinal is then transferred to the RPE cells to undergo its own recycling cycle. 
The second messenger of the phototransducion cascade is cGMP. cGMP is synthesized from GTP by guanylyl cyclase (GCy) and broken down to 5'-GMP by cGMP phosphodiesterase.

cGMP-phosphodiesterase is an enzyme controlled by the visual pigments. The structural change of rhodopsin induces activation of the G-Protein transducin $(\mathrm{G})$. Two activated molecules of transducin activate cGMP-phosphodiesterase (PDE $\left.{ }^{* *}\right)$.

$\mathrm{PDE}^{* *}$ hydrolyses cGMP into 5'-GMP $(\beta)$. The reduction of cGMP allows the cGMP gated sodium channels to close, preventing influx of sodium and causing hyperpolarization of the photoreceptor.

In darkness, when the photoreceptor is in a depolarized state, glutamate is released from the synaptic terminal via ribbon synapses into the synaptic cleft. On a light stimulus, when the photoreceptor is hyperpolarized, neurotransmitter release stops and the following cells react in different ways. The description of the events following reduction of glutamate release will be described in later sections. 


\subsubsection{Reverting to the dark-current state}

Several negative feedback mechanisms are used by the photoreceptors to revert to the dark current state after a flash of light. On a light stimulus, the intracellular concentrations of cGMP and calcium are reduced. While cGMP directly controls the opening and closing of the CNG channels, changes in calcium concentration in the PR indirectly trigger negative feedback mechanisms (Komolov et al., 2009) leading to an increase in intracellular cGMP concentration.

The enzyme guanylyl cyclase synthesizes cGMP from GTP. Guanylyl cyclase is regulated by the calcium-dependent guanylyl cyclase activating protein (GCAP). The cGMP sensitive channels mediate influx of calcium ions next to the influx of sodium ions. In darkness, calcium concentration in the PR OS is relatively high and guanylyl cyclase is partly inhibited. On a light stimulus, when the intracellular calcium concentration is reduced, guanylyl-cyclase is gradually activated. As a result of the increase in cGMP concentration, the cGMP sensitive channels open again. This way, guanylyl cyclase helps to restore the depolarized state of the plasma membrane by supporting the opening of cGMP channels (Kandel et al., 2000).

In darkness, when the calcium concentration in the PR is relatively high, the calcium binding protein recoverin is normally bound to the enzyme rhodopsin kinase. Thereby, rhodopsin kinase is kept inactivated (Ames et al., 2006). Thereby, in high calcium concentration, recoverin inhibits rhodopsin phosphorylation. On a light stimulus, when calcium concentration in the PR OS is reduced, recoverin dissociates from rhodopsin kinase (RK) (Kawamura, 1993) and free RK phosphorylates the cytosolic tail of activated rhodopsin. This way, rhodopsin is inactivated and can no longer activate transducin, which in turn can no longer activate PDE. Again, the intracellular concentration of cGMP increases.

Furthermore, the protein arrestin binds to the phosphorylated rhodopsin tail to further inhibit the activity of rhodopsin (for further reading on arrestin-mediated regulation of activated rhodopsin see e.g. Gurevich EV and Gurevich VV, 2006). 


\subsubsection{Synaptic transmission in the retina}

The photoreceptors synapse with bipolar and horizontal cell dendrites in the OPL. Rods contact ON rod-BCs, cones contact ON and OFF cone-BCs (Hack et al., 1999). The neurotransmitter of the photoreceptors, the bipolar cells and the ganglion cells is the excitatory amino acid transmitter glutamate. The inhibitory systems (namely horizontal and amacrine cells) use mainly GABA and glycine.

\subsubsection{Excitatory transmission - Glutamatergic ribbon synapses}

Ribbon synapses are specialized synapses for transducing sensory signals with high fidelity across a broad range of stimulus intensities and for long periods of time. They are exclusive to synapses requiring continuous and graded depolarization and keep up sustained calciumdependent release rates of neurotransmitter substances. This way, they are able to sense and code for rapid changes in sensory signals (Matthews and Fuchs, 2010). In the retina, photoreceptors and bipolar cells are equipped with ribbon synapses which transfer information through the neurotransmitter glutamate (tom Dieck and Brandstatter, 2006). The ribbon synapses of the photoreceptors lie in the OPL, the ribbons of the bipolar cells lie in the IPL.

The ribbon is a plate-like structure with a large surface that is anchored to the presynaptic membrane and extends into the presynaptic cytoplasm. The ribbon is attached to the presynaptic plasma membrane by a "leash". The ribbon is this way able to float in the cytoplasm. Molecularly, it represents a multi-protein-complex. A number of proteins have been identified as components of the ribbon itself, its cytoplasmic surround and its anchoring to the plasma membrane. RIBEYE is the only known component specific to ribbon synapses (Schmitz et al., 2000). Multiple RIBEYE-RIBEYE interactions have been shown to build the synaptic ribbon and anchor it via interactions with the anchoring protein Bassoon (Magupalli et al., 2008, tom Dieck et al., 2005). The anchoring protein Bassoon is present in the photoreceptor ribbon synapses at the OPL. At the IPL, bassoon was shown to be present in the GABAergic conventional synapses of the amacrine cells. Bassoon was not found in the bipolar cell ribbons in the IPL (Brandstatter et al., 1999). Dick, O. et al. in 2001 showed that Bassoon and Piccolo, both presynaptic scaffolding proteins involved in the ribbon complex, are present presynaptically in glutamatergic ribbon synapses and in conventional GABAergic and glycinergic synapses. Although the two proteins were coexpressed in all photoreceptor ribbon synapses and in some conventional amacrine cell synapses, Bassoon was not present in bipolar cell ribbon synapses. In 2003, Dick, O. et al. found out that absence of bassoon prevents anchoring of the photoreceptor ribbon to the presynaptic active 
zone during synaptogenesis. This resulted in impaired PR synaptic transmission, formation of ectopic synapses and impairment of dendritic differentiation of postsynaptic neurons.

Numerous vesicles are tethered to the ribbon. About 130 vesicles are tethered to a ribbon compared to about 50 vesicles at a conventional synapse (Rao-Mirotznik et al., 1995). The ribbon has also been reported to act as a "conveyor-belt", which supplies the synaptic complex continuously with readily-releasable vesicles (reviewed by Lenzi and von Gersdorff, 2001). Neurotransmitter release from ribbon synapses has been reported described by two different kinetic models. The vesicles located closest to the plasma membrane below the synaptic ribbon, which are not tethered to the ribbon are released immediately. Slower release was found for the many vesicles tethered to the ribbon itself (reviewed by Matthews and Fuchs, 2010). Knockout of Bassoon/disruption of ribbons in cochlear hair cells eliminated the fast release component (Khimich et al., 2005).

At conventional synapses, only few vesicles are readily releasable. The majority is immobilized in reserve attached to the cytoskeleton by synapsins (Pieribone et al., 1995). Synapsins are believed to regulate neurotransmitter release via bridging between synaptic vesicles and cytoskeletal elements (e.g. actin) depending on their phosphoryation state (Greengard et al., 1993). Pieribone et al. in 1995 showed, that two distinct pools of synaptic vesicles exist, one of which contains synapsin. Depletion of synapsin caused a marked depression of neurotransmitter release following high-frequency stimuli. They concluded, that the synapsin-associated vesicle pool is required to sustain NT release on high-frequency stimuli. However, ribbon synapses of photoreceptors and bipolar cells do not contain synapsins (Mandell et al., 1990). Analyses of vesicle pools and vesicle turnover have shown that there is no need for a synapsin-like function at ribbon synapses (Holt et al., 2004). They have even been suggested to substitute themselves for synapsin. At ribbon synapses, the majority of vesicles is highly mobile in the cytoplasm and not tethered to synapsins or the actin cytoskeleton. This is important for continuous vesicle supply of the ribbon and thus continuous exocytosis (Rea et al., 2004). Photoreceptor ribbon synapses are able to adjust the release of glutamate to changing inputs, optimising information transfer onto the postsynaptic horizontal and bipolar cells. Changes in stimulus intensity are encoded by changes in tonic rate of transmitter release (von Gersdorff, 2001; Parsons and Sterling, 2003). 


\subsubsection{Inhibitory transmission - GABA and Glycine receptors}

Inhibition by the neurotransmitter substances GABA and glycine shapes retinal responses to a light stimulus. Initial inhibitory modification of the photoreceptor signal happens in the OPL mediated by horizontal cells. The second inhibitory modulation of the signal is mediated by amacrine cells at the IPL level. Inhibitory mechanisms in the end contribute to the so-called center-surround organization of the ganglion cells detecting changes in lighting conditions and contrasts (Kolb, 2006), which is up to date not fully understood. The retina contains several types of glycine and GABA-receptors. The diversity and distribution of these receptors within the many retinal neurons is very large (Grunert, 1999; Wassle et al., 1998). Only a gross overview can be given in this context.

$\mathrm{GABA}_{\mathrm{A}}$ and $\mathrm{GABA}_{\mathrm{C}}$ receptors in the IPL are ionotropic receptors transferring GABAergic signals (Wassle et al., 1998). They are ligand-gated integral membrane channels permeable to chloride ions and composed of a variety of subunits. The respective subunit-composition depends on the location of the receptor (Wassle et al., 1998). The $\mathrm{GABA}_{\mathrm{A}}$ receptors are located postsynaptically in the dendrites of amacrine and ganglion cells and at the axon terminals of bipolar cells. $\mathrm{GABA}_{\mathrm{C}}$ receptors are preferentially located at the axon terminals of bipolar cells (reviewed by Koulen et al., 1998a). In the IPL of the mouse retina, they have been reported on rod bipolar cell axon terminals (McCall et al., 2002). Rod BCs express different types of $\mathrm{GABA}_{\mathrm{A}}$ and $\mathrm{GABA}_{\mathrm{C}}$ receptors in the IPL (Fletcher, 1998). The BCs receive synaptic input at their axon terminals from $\mathrm{GABAergic} A C s$ via $\mathrm{GABA}_{\mathrm{A}}$ receptors.

$\mathrm{GABA}_{C}$ receptors are more sensitive to $\mathrm{GABA}$ than $\mathrm{GABA}_{\mathrm{A}}$ receptors. The response mediated by $\mathrm{GABA}_{\mathrm{A}}$ receptors is brief, with fast rise and decay time course, whereas the $\mathrm{GABA}_{\mathrm{C}}$ response is prolonged, with slower rise and decay time courses (Feigenspan and Bormann, 1994). These two receptors are distributed differentially and create the right setting for a dynamic response that is adaptable to different lighting conditions (Dong and Werblin, 1998; Freed et al., 2003). $\mathrm{ON}$ rod bipolar cells respond slowest and their response is mainly mediated via $\mathrm{GABA}_{\mathrm{C}}$ receptors. OFF cone bipolar cells answer fastest and this response is mainly mediated via $\mathrm{GABA}_{\mathrm{A}}$ receptors (Lukasiewicz et al., 2004).

$\mathrm{GABA}_{\mathrm{B}}$ receptors are G-Protein-coupled (metabotropic) receptors that regulate potassium or calcium channels (Slaughter, 1995) via second-messenger systems (Wassle et al., 1998). GABA receptors are found in the OPL. There, they lie presynaptically in horizontal cell processes invag- 
inating into photoreceptor terminals. In the IPL, $\mathrm{GABA}_{\mathrm{B}}$ receptors are present presynaptically in amacrine cells, as well as postsynaptically in amacrine and ganglion cells (Koulen et al., 1998b).

Glycine receptor (GlyR) immunolabeling by Wassle et al. in 1998 showed strong postsynaptic immunofluorescence in the IPL. No immunostaining for GlyR was found at photoreceptor terminals. Staining in the outer IPL was attributed to glycinergic synapses through which the rod signal passes from AII amacrine cells to OFF cone bipolar cells (see "Synaptic transmission in the IPL"). The puncta in the IPL could be located to the dendrites of ganglion cells. In 2009, Mørkve and Hartveit reported evidence of possible glycinergic inhibition from amacrine cells onto rod bipolar axon terminals. They stated, this inhibitory input is likely to suppress exocytosis from rod bipolar cells. 


\subsubsection{Synaptic transmission in the OPL}

The small terminals of the rod photoreceptors (rod spherules) usually contain a single ribbon with a long active zone that bends around four invaginating postsynaptic elements (RaoMirotznik et al., 1995). The four postsynaptically invaginating processes are dendrites of bipolar cells and processes of horizontal cells. Cone synaptic terminals (cone pedicles) contain several ribbons with shorter active zones. They are also contacted by dendrites of bipolar cells and horizontal cells (tom Dieck and Brandstatter, 2006).

In the non-stimulated state (in darkness), photoreceptors have a depolarized membrane potential and spontaneously release neurotransmitter. All photoreceptors hyperpolarize in response to light and transmit their signal onto bipolar cells. Different types of bipolar cells process photoreceptor input in different ways. The bipolar cells are either hyperpolarized (OFF-bipolar) or depolarized (ON-bipolar), depending on their type of glutamate receptor. The BCs express different types of glutamate receptors. OFF-BCs make contacts using excitatory ionotropic glutamate receptors (iGluRs). These bipolar cells receive excitatory glutamatergic input from the photoreceptors in darkness. Upon light and consecutive neurotransmitter release reduction, they respond like the photoreceptor with a hyperpolarizing (sign-preserving or OFF-) response. Neurons within the OFF-pathway (horizontal cells, OFF-bipolar cells, amacrine cells and ganglion cells) express iGluRs. (Connaughton, 2007 and Kolb, 2006). ON-BCs express inhibitory glutamate receptors (metabotropic glutamate receptors/mGluRs). Via the mGluRs, these bipolar cells get inhibitory glutamatergic input in darkness. When neurotransmitter release is reduced by light, they show a depolarizing (sign-inverting or ON-) response (Kolb, 2006). mGluRs are coupled to G-proteins. Glutamate binding onto mGluRs can have a variety of effects depending on the second messenger cascade to which the receptor is coupled (Connaughton, 2007). Further, one mGluR subtype was found on photoreceptor terminals contacting ON-BCs. They have been shown to downregulate intracellular calcium concentration in PR terminals and thus mediate an inhibitory feedback loop at the photoreceptor synapse (Koulen et al., 1999).

The bipolar cells represent the stage of retinal signal processing where ON- and OFF- signals are established (Molnar and Werblin, 2007). iGluR- and mGluR-expressing bipolar cells represent parallel visual pathways for contrast detection. These are known as OFF (dark-on-light) and ON (light-on-dark) pathways. Cones contact both iGluR- and mGluR-driven cone bipolar cells. On the contrary, rods only contact mGluR-driven rod bipolar cells. 
At the OPL-level, horizontal cells are further involved in contrast enhancement. Horizontal cells receive synaptic input from photoreceptors and then feed back onto other photoreceptors. Mouse horizontal cells receive cone input at their dendritic branches, whereas they receive rod input at their axon terminals (Peichl and González-Soriano, 1994; Pan and Massey, 2007).

Horizontal cells are depolarized by glutamate released from PR synaptic terminals in darkness. They contact the photoreceptors at the photoreceptor ribbon synapse and receive excitatory input via postsynaptic iGluRs. The horizontal cell itself releases inhibitory neurotransmitter upon depolarization. Wassle et al. in 1998 described that horizontal cells contain a GABA-synthesizing enzyme and release GABA. Deniz et al. in 2010 reported hints that mouse horizontal cells may be atypical GABAergic interneurons, with no GABA uptake, but a glutamate and/or glutamine transport system allowing GABA synthesis from glutamate or glutamine.

On light activation (glutamate cut-off), the membrane potential of the horizontal cell changes from depolarisation to hyperpolarization. Thus, on light activation, inhibition mediated by the horizontal cells in darkness is decreased. This causes depolarization of the contacted photoreceptors (lateral inhibition). This way, the horizontal cells add and modify signals from photoreceptors resulting in what is known as the center surround organization of the following bipolar cell. Horizontal cells modulate the photoreceptor signal under different light conditions and shape the receptive field of the bipolar cell response in the means of OFF- and ON-centers (Kolb, 2006) 


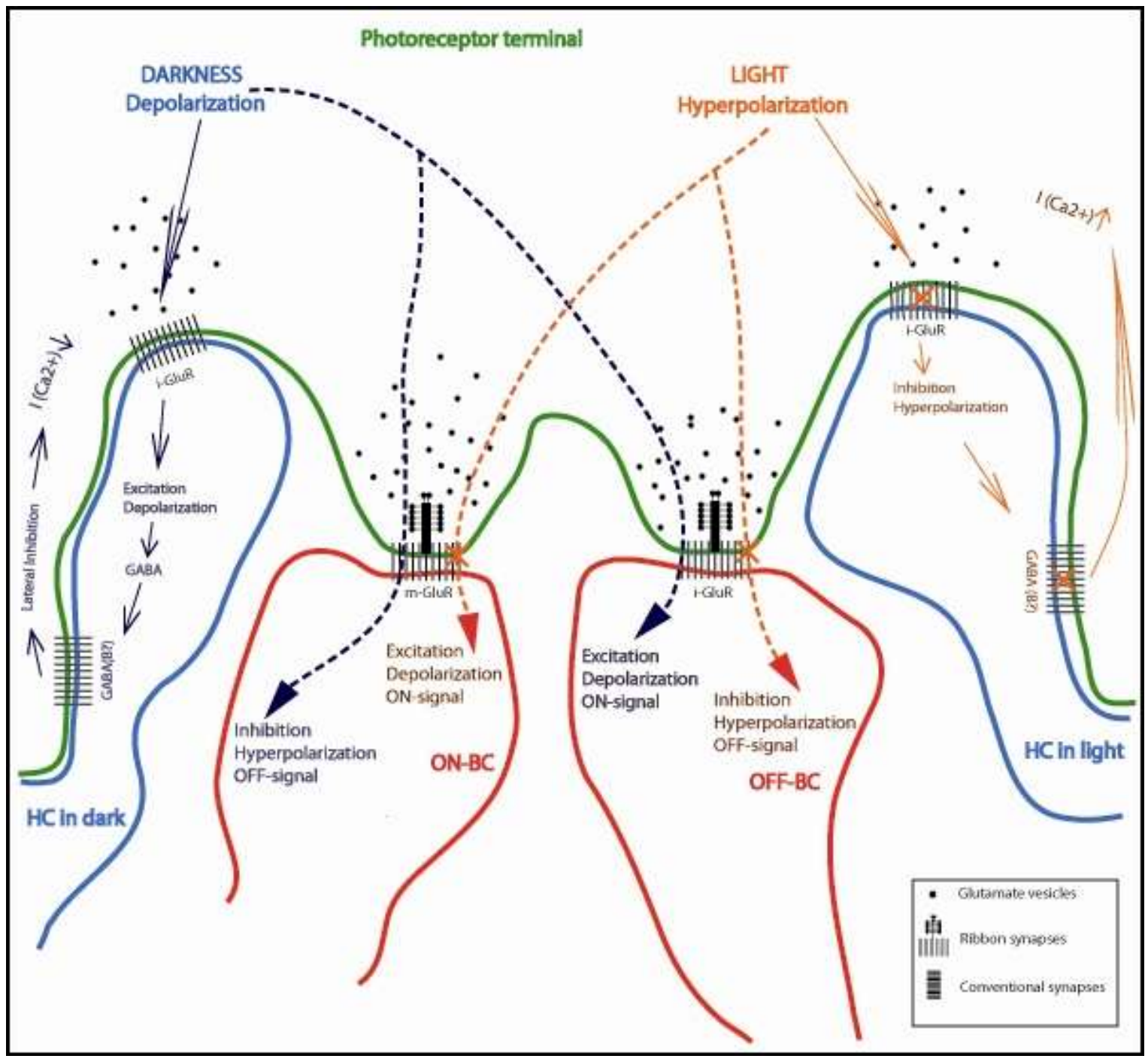

Figure 3: Schematic of iGluR- and mGlur-mediated synaptic transmission at the OPL level of the retina

(freely adapted by the author from Kolb, 2006, p.56)

At the OPL level, ON and OFF signals are established due to differential expression of receptors on BCs. ON BCs possess mGluRs, while OFF-BCs are equipped with iGluRs. Effects of light and darkness, respectively, are shown for both types of receptors. In vivo, the constellation shown in this picture does not exist. The PR terminal contacts either an $\mathrm{ON}$ or an OFF BC.

Events at the mGluR-containing ON-BC: In darkness, glutamate containing vesicles are steadily released from the PR. Because of the inhibitory characteristics of the mGluR, this causes hyperpolarization of the BC, establishing an OFF-signal. Stimulation by light cuts off glutamate release from the PR terminal, causing depolarization of the BC via the mGluR, establishing an ON-signal.

Events at the iGluR-containing OFF-BC: In darkness, glutamate is steadily released from the PR terminal. Due to the excitatory characteristics of the iGluR, this causes depolarization of the BC, establishing an ON-signal. Stimulation by light causes glutamate-cutoff from the PR terminal, resulting in hyperpolarization of the $\mathrm{BC}$, establishing an OFF-signal.

Also shown in this picture are the horizontal cells at the OPL, putative of mediating lateral feedback onto photoreceptors. The mechanisms of these lateral feedback loops are up to now not fully understood. Involvment of GABA (B?) receptors modifying calcium currents are discussed. 


\subsubsection{Synaptic transmission in the IPL}

The rod and cone bipolar cell terminals contain several small ribbons that are opposed by noninvaginating postsynaptic processes of amacrine and ganglion cells.

The bipolar, amacrine and ganglion cells synapse in the IPL. The IPL consists of two sublaminae. The distal sublamina (OFF-sublamina) contains synapses between iGluR-bearing OFF-BCs and OFF GCs. In the proximal sublamina (ON-sublamina), mGluR-bearing ON-BCs contact ON GCs.

Amacrine cells take over similar functions in the IPL as the horizontal cells do in the OPL. The amacrine cells receive synaptic input from the bipolar cell synaptic terminals and send their axons laterally within the IPL to contact other BCs and GCs. They have been shown to add information from the $\mathrm{BCs}$ to the GC-receptive field organization, the system for contrast enancement. Amacrine cells are about equally divided into glycine- and GABA-releasing cells.

Cones contact cone BCs, which directly converge their signals onto the accordant ganglion cells. Rods contact the mGluR-driven ON-rod BCs, which do not synapse directly with ganglion cells. The rod pathway takes a little side-step, as the rod bipolars use two types of amacrine cells as intermediaries to get rod information to ganglion cells. The two types of amacrine cells best characterized up to now are the glycinergic AII cell and the GABAergic A17 cell.

AII cells are small-field amacrines linking the rod and cone pathways so that the rod signals can use the cone bipolar pathway to reach the ganglion cells (Dacheux and Raviola, 1986). The AII cell passes rod information either via gap junctions to ON cone bipolar cells that then excite ON ganglion cells or via chemical synapses to the OFF cone bipolar and then the OFF ganglion cells. A17 cells are wide-field amacrine cells collecting signals from rod bipolar cells. They interconnect rod bipolar cells by reciprocal synapses. Presumably, the A17 is an integrating unit that helps set sensitivity levels over a large area of rod photoreceptors and rod bipolar cells (Nelson and Kolb, 1985). The A17-mechanisms are not yet completely clarified. The GABAergic A17 cell uses a $\mathrm{GABA}_{\mathrm{C}}$ receptor to feed back onto rod bipolar cell axons. GABAergic amacrine cells connect to neighboring homologous amacrine cells by gap junctions. Most GABAergic amacrine cells contain at least one other neuroactive substance besides GABA. The variety of neuroactive agents may influence the organization of the ganglion cell receptive field and modulate perception under changing lighting conditions. 


\subsection{The electroretinogram (ERG)}

When the retina receives a light stimulus, many different cells get activated at the same time resulting in currents that are large enough to initiate a change in trans-ocular voltage. This voltage can be recorded from the cornea as the electroretinogram (ERG). So-called "Retinal action potentials" were first discovered by the Swedish physiologist Frithiof Holmgren in 1865. In 1933, the Swedish physiologist Ragnar Granit described the single components of what he then called "Retinal flash-response curve". His experiments were carried out on decerebrated cat heads fixed in a shielded and grounded black box and adjusted with one eye towards an opening of a tube leading to the stimulus source. As corneal electrodes, he used silver-silverchloride $(\mathrm{Ag} / \mathrm{AgCl})$ wires placed onto the cornea with cotton wicks dipped into Ringer's solution as a moistening conductant. He also performed experiments on the time course of the diminishing of the signal components. During progressive narcotization of live experimental animals, he tested the pharmacological influence of inhalative ether. Granit described the three main components of the "retinal action potential" and named them PI-PIII in order of their diminution by inhalative ether.

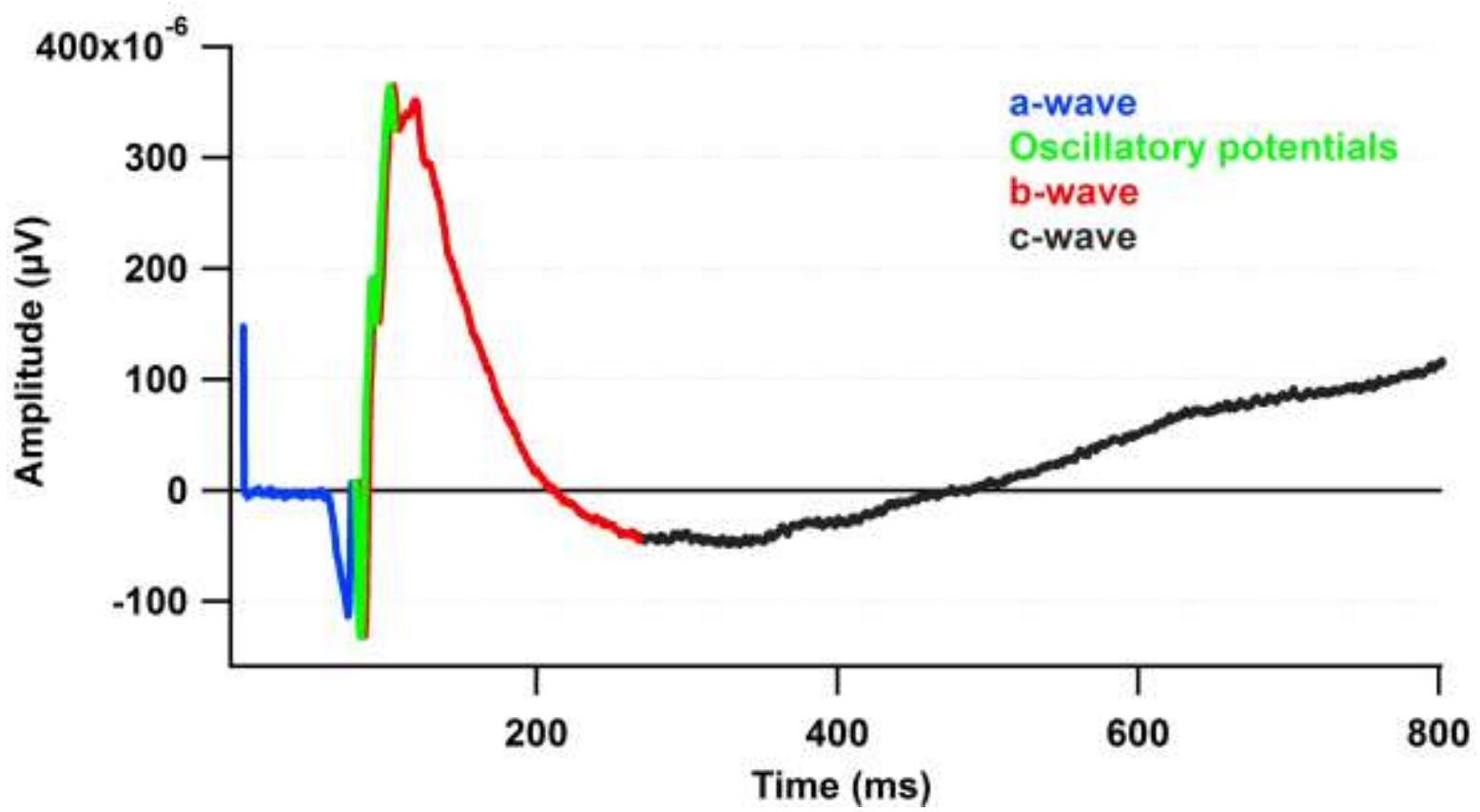

Figure 4: Typical scotopic mouse ERG

Fig.4 shows a standard ERG trace of a Neuroligin 2 wildtype mouse. All components described in this section can be clearly seen. A-wave (blue), oscillatory potentials (green) on the rising part of the b-wave (red) and the beginning of the c-wave (black). This trace was recorded at a stimulus length of 5ms, attenuation level 10, ISI of $2 \mathrm{sec}$ and averaged ten times. 
Component PI is a positive potential component driven by a large-field-illumination and high light-intensity, which rises slowly to its maximum within 2 seconds after the stimulus. This component is termed the c-wave. It is an inhomogeneous component whose prevalence varies greatly between different species. The c-wave is mentioned here for completeness reasons, but is not included in our analysis of the ERG. The second component he described (PII) is a rapidly rising and rapidly falling positive wave. It is the only process of the retinal action potential that Granit detected at all suprathreshold intensities. PII is selectively affected by asphyxia of the experimental animal (occlusion of the carotid artery). It further diminishes gradually in the course of prolonged ether anaesthesia. This component is termed the b-wave. The third component (PIII) is of negative value. It is, just like PI, a response observed at high-intensity stimuli. It is the last component affected by continued ether anaesthesia. PIII is termed the a-wave.

\subsubsection{The a-wave}

The a-wave is a negative-value component of the ERG that originates from the photoreceptors and emerges only when stimuli reach higher intensities. Robson et al. in 2003 showed that the photoreceptor current was the only significant component of the leading edge of the a-wave in the macaque monkey ERG. The PR dark current produces a corneal-positive potential. Penn and Hagins in 1969 studied the spatial distribution of the membrane current of rods in the rat retina. They concluded that suppression of the dark-current by stimulation with light suppresses the positive potential and the negative-going a-wave emerges. Hood and Birch in 1990 argue, that intracellular changes in cGMP on light stimulation are reflected by a linear course of the a-wave over a wide range of intensities. They explain a following non-linear course of the a-wave by the limited number of conductance channels that depend on cGMP concentration. Barraco et al. in 2006 stated a strict connection of the ERG a-wave with early photoreceptoral activities by fitting recorded a-waves with computational models of physiological behaviour which would take place in early stages of phototransduction. Vinberg et al. in 2009 proposed, based on the results of aspartate-isolated ERGs, that the leading edge of the a-wave of the ERG corresponds to the outer segment light-sensitive current, but also a "nose"-like component of the a-wave that is attributed to rod inner segment activity on light stimulation.

\subsubsection{The b-wave}

Intraretinal microelectrode recordings from cat eyes by Arden and Brown were published in 1965. The maximum amplitude of the b-wave was slightly distal to the INL and accordingly proximal to the photoreceptors. By blocking retinal synaptic transmission proximal to the photo- 
receptors, the b-wave was abolished, but the a-wave was left unaffected, excluding PRs as source of the b-wave. Miller RF and Dowling in 1970 suggested that light-evoked variations in extracellular $\mathrm{K}^{+}$concentration $\left[\mathrm{K}^{+}\right]_{\mathrm{o}}$ induce a current through Muller cells. A light-induced $\mathrm{K}^{+}$influx into the distal part of the Muller cells would depolarize the Muller cells and drive an equal $\mathrm{K}^{+}$efflux from more proximal regions of the cell. The return current flowing through extracellular space from the proximal to the distal retina would generate the positive b-wave. However, later studies lead away from the Muller cells as primary generators of the b-wave. Dick E et al. in 1985 found two separable sources of light-evoked increases in $\left[\mathrm{K}^{+}\right]_{\mathrm{o}}$. They stated that the b-wave is closely associated with depolarizing bipolar cells. ON BC processes in the OPL generate a $\mathrm{K}^{+}$efflux at light onset that can be detected as the distal $\left[\mathrm{K}^{+}\right]_{\mathrm{o}}$ increase. This $\left[\mathrm{K}^{+}\right]_{\mathrm{o}}$ modulation depolarizes Muller cell processes and initiates the transretinal current associated with the b-wave. In this model, the b-wave would primarily represent a second-order signal of depolarizing bipolar cell activity. Gurevich L and Slaughter in 1993 showed that the b-wave is a phasic signal and resembles the light response waveform of ON bipolar cells. Xu and Karwoski (1994a, b) performed current source density analyses of ERG depth profiles. Blocking $\mathrm{K}^{+}$influx into Muller cells by $\mathrm{Ba}^{+}$did not effectively abolish the b-wave, indicating that the primary $b$-wave generators are not Muller cells. In summary, literature indicates that the cornea-positive b-wave is primarily related to depolarizing ON-bipolar cells.

\subsubsection{The c-wave}

The c-wave (PI) is usually of positive voltage. It consists of two subcomponents. A cornealnegative subcomponent (termed "slow PIII)" is generated by the Muller cells and a cornealpositive subcomponent is generated by the RPE (Frishman, 2006). It is likely that both of the cwave subcomponents occur because of a light-evoked decrease in extracellular potassium concentration $\left[\mathrm{K}^{+}\right]_{\mathrm{o}}$ (Oakley and Green, 1976). The RPE forms an apical and a basal membrane, which are electrically incompletely separated by tight junctions enclosing the single cells (characterized as the R-membrane, Brindley and Hamasaki 1963). The RPE component of the c-wave and the hyperpolarization of the apical RPE membrane show time courses similar to the lightevoked, $\left[\mathrm{K}^{+}\right]_{\mathrm{o}}$ decrease (Miller SS and Steinberg 1977).

"slow PIII" is a Muller cell contribution to the c-wave. The Muller cells function as " $\mathrm{K}^{+}-$ transporters". Membrane hyperpolarization in the retina causes a reduction of $\left[\mathrm{K}^{+}\right] \mathrm{o}$. Via a $\mathrm{Na}^{+}-$ $\mathrm{K}^{+}$ATPase, $\mathrm{K}^{+}$enters the Muller cells and is radially carried intracellularly to regions of lower 
$\left[\mathrm{K}^{+}\right]_{\mathrm{o}}$. Dick, E. et al. showed in 1985 that the time course of the Muller cell hyperpolarization was similar to the light-evoked reduction in $\left[\mathrm{K}^{+}\right]_{\mathrm{o}}$ and the slow PIII, respectively.

\subsubsection{The Oscillatory Potentials}

In 1952, Cobb and Morton described rapid oscillations on the rising part of the b-wave. They could be provoked by a short duration, high-intensity flash. Brown in 1968 described highamplitude OPs in the periphery of the retina and lower amplitudes towards the fovea. He also stated that the oscillatory wavelets were critically dependent on retinal circulation, as they were abolished, when retinal blood supply was occluded. His intraretinal depth recordings located the maximum amplitudes of the OPs at about $16 \%$ depth from the proximal end. More distal retinal cell layers, like the PR and BC layer were thus unlikely the origins of the OPs. Ogden in 1973 recorded the maximum amplitudes of the rapid OPs at the level of the IPL. Later, in 1977, Foerster et al. confirmed this statement. They showed that the frequency characteristics of horizontal cells are too slow to be the generators of the OPs. The same reason excluded the Muller cells as possible generators of the OPs (Ogden, 1973). Instead were the axon terminals of the bipolar cells, the processes of the amacrine cells and the dendrites of the ganglion cells then taken into focus. A possible amacrine cell contribution to the rapid oscillatory potentials was confirmed again in intraretinal depth recordings by Wachtmeister and Dowling in 1978. They located the origin of the rapid oscillations to the layer of amacrine cells.

In addition, ganglion cell rhythmic discharge was suggested as possible generator of the OPs (Steinberg, 1966; Ogden, 1973). Vaegan and Millar in 1994 showed that pharmacologically induced damage to the GC dendrites diminished the oscillatory response in the retina. Pharmacological disruption of GABA-mediated pathways (Wachtmeister, 1980) selectively diminishes the OPs. The rapid oscillations are more affected than later oscillations. These findings of reduced OPs in GABA-deficient retinae may indicate disturbances in the inhibitory feedback part of the retinal electric circuitry related to the ON-pathway of the retina and initiated by amacrine cells. Later OPs are more sensitive to the blocking of glycinergic pathways (Korol et al., 1975; Wachtmeister, 1980). However, Wachtmeister in 1998 reviewed, that the responses from tangential dipole cells as the amacrines cannot be measured at the cornea (thus the ERG) and that the amacrines could so not be the only generators of the rapid OPs. Amacrine calls may initiate a series of neuronal signaling underlying the rapid OPs. 


\subsection{Investigated Proteins}

\subsubsection{Neuroligins}

Neuroligins are postsynaptic transmembrane proteins that derive from at least four genes (Bolliger et al., 2001; Ichtchenko et al., 1996). Intracellularly, neuroligins bind to the postsynapticdensity component PSD-95 (Irie et al., 1997), a scaffolding protein in excitatory synapses. Extracellularly, neuroligins bind to $\beta$-neurexins. Neuroligins resemble cell surface receptors (Ichtchenko et al., 1995).

Neurexins are located presynaptically and associate with synaptic vesicles by interaction with presynaptic scaffolding proteins (Biederer and Sudhof, 2000). Scheiffele et al., 2000 suggested the neurexin-neuroligin link may act as a trans-synaptic bridge bringing vesicles into alignment with the postsynaptic density. The $\beta$-neurexin-neuroligin-link is present at glutamate synapses. Graf et al. in 2004 showed that during synaptogenesis, neurexin alone is sufficient to induce glutamate and GABA postsynaptic differentiation. Conversely, neuroligins induce presynaptic differentiation in both glutamate and GABA axons. Neuroligins-1, -3 and -4 localize to glutamate postsynaptic sites, whereas neuroligin-2 localizes primarily to GABA synapses. Neurexins and neuroligin-2 are involved in GABAergic synaptogenesis.

Synaptogenesis involves two processes (Serafini, 1999; Yamagata et al., 2003). Initially, contact between the growing axon and the target neuron must be made and then the differentiation must be initiated by assembly and stabilization of pre- and postsynaptic proteins. Specific intracellular binding sites are necessary for the proteins that mediate protein recruitment during synaptogenesis. Neuroligins are required for proper synapse maturation and function, but not for the initial formation of synaptic contacts (Varoqueaux et al., 2006). Loss of NLs 1-3 in triple knockout brains causes a decrease in spontaneous GABAergic and glycinergic activity and reduction of spontaneous glutamatergic activity in the mouse respiratory brain stem. In the affected cells, postsynaptic recruitment of $\mathrm{GABA}_{\mathrm{A}}$ receptors is altered. The total synapse number was not affected, which indicated that the NLs determine functional rather than structural parameters of synapses by regulating the recruitment of synaptic proteins. The deletion of NLs 1-3 affects inhibitory synaptic transmission more strongly than excitatory transmission (Varoqueaux et al., 2006). In 2010, Lui et al. showed by immunolabeling that Neuroligin 2 is also located presynaptically at the ribbon synapses of photoreceptor terminals. 


\subsubsection{Protein 4.1}

In 1980, Feo et al. described the absence of an erythrocyte membrane protein (named band 4.1) in a patient suffering from severe familiar elliptocytic anaemia. They proposed a role of protein band 4.1 in maintaining the erythrocyte membrane stability. In 1979, Ungewickell et al. showed an in vitro formation of a complex between spectrin, f-actin and protein 4.1. They stated that band 4.1 is required for the formation of the spectrin-actin complex and that the absence of protein 4.1 could be responsible for the weakening of the spectrin-actin interaction and thus for the instability of the erythrocyte membrane. Fowler and Taylor (1980) showed that band 4.1, spectrin, and actin are all required for the optimal formation of skeletal supramolecular structures. Cohen and Foley (1980) suggested that band 4.1 may play an important role in promoting the anchoring of actin filaments to the cytoplasmic surface of the erythrocyte by spectrin. Tanaka et al. in 1991 proposed that the spectrin-actin interaction is regulated $\mathrm{Ca}^{2+}$-dependently by a protein 4.1-calmodulin complex. They wrote that erythrocytes undergo changes in shape in response to increased influx of calcium ions and suggested that $\mathrm{Ca}^{2+}$ may play a role in modulating the deformability of the erythrocyte membrane by affecting the interactions of membrane and cytoskeleton. Their results showed that protein 4.1 alone can potentiate the actin cross-linking and actin binding activities of spectrin, but cannot confer $\mathrm{Ca}^{2+}$-sensitivity on the spectrin-actin interaction. The binding of protein 4.1 to calmodulin is not $\mathrm{Ca}^{2+}$-dependent, but the complex confers $\mathrm{Ca}^{2+}$-sensitivity on the spectrin-protein 4.1-actin interaction. They proposed that this mechanism is in part responsible for controlling erythrocyte shape during changes in cytoplasmic $\mathrm{Ca}^{2+}$ concentration. Kelly et al. in 1991 established a calmodulin binding site within the N-terminus of protein 4.1. They implicated a possible calmodulin regulation of the cytoskeleton during differentiation and development. In 1998, Peters et al. characterized a set of four paralogous 4.1 genes that have been evolutionary conserved in rodents and primates. In addition to the prototypical red blood cell 4.1R (human gene symbol: EPB41,) two homologues are strongly expressed in the nervous system and brain $(4.1 \mathrm{~N}, E P B 41 L 1$; and 4.1B, EPB41L3) and one is widely expressed in many tissues $(4.1 \mathrm{G}, E P B 41 L 2)$. The gene sequence encoding for protein $4.1 \mathrm{G}$ was established by Parra et al. in 1998. After the protein 4.1R gene, they discovered a second 4.1 gene which is widely expressed among human tissues and which exhibits three regions of high homology to 4.1R. These regions are the membrane binding domain, the spectrin-actin binding domain and the $\mathrm{C}$-terminal domain. Interspersed among these shared domains are unique sequences that may 
define functional differences between 4.1R and 4.1G. Specific isoforms of 4.1R and 4.1G exhibit differential subcellular localizations. 


\section{Materials and Methods}

\subsection{Animals}

The NL2 KO mice were kindly provided by Dr. Frédérique Varoqueaux of the Max-Planck Institute for Experimental Medicine, Dept. of Molecular Neurobiology, Göttingen, Germany.

The Protein 4.1 B/G/N triple knockout mice were generated and kindly provided by Dr. phil. nat. Aleksandra Ivanovic of the Max-Planck Institute for Experimental Medicine, Dept. of Molecular Neurobiology, Göttingen, Germany.

For the studies presented here, we tested NL2 single knockout and Protein $4.1 \mathrm{~B} / \mathrm{G} / \mathrm{N}$ triple knockout mutant mice in comparison with their wild type littermates at the age from 6-10 weeks. All animal experiments were performed in compliance with the guidelines for the welfare of experimental animals issued by the Federal Government of Germany and approved by the animal care committee of the Göttingen University Medical Center and the animal care office of the State of Lower Saxony.

Genotyping was performed on all animals prior to investigations and repeated after the experiments. The DNA was extracted from small tail biopsies $(\sim 5 \mathrm{mg})$. The results of the preexperimental genotypings were documented on a list that was not handed out to the investigator prior to the experiments. The animals were chosen randomly for the experiments. They were treated as numbers encoded by special earmarks. Only after the experiments were completed, the animals were segregated into knockout and wild type according to the list. One putative NL2-KO animal was eliminated from the analysis because post-experimental genotyping discovered a heterozygous genotype. 


\subsection{Electroretinograms - ERG}

\subsubsection{Animal procedures}

Before the ERG experiments, animals were dark adapted overnight for at least twelve hours. All preparatory procedures were performed under dim, red light.

Mice were anaesthetized by intraperitoneal injection of ketamine $(0.125 \mathrm{mg} / \mathrm{g})$ and xylazine $(2.5 \mu \mathrm{g} / \mathrm{g})$. To assure as little disturbance of the animal as possible, an intraperitoneal line (24 Gauge) was left connected to a syringe filled with additional narcotic solution. Mice were laid onto their stomach on a heating plaid to assure constant body temperature of $37^{\circ} \mathrm{C}$. Temperature control was performed by a rectal thermometer. Stable anaesthesia of the animal was controlled by carefully watching EEG and ECG on an oscilloscope. In sufficient narcotization, EEG amplitudes were low and the baseline was stable. On the fading out of the narcotic medication, larger EEG amplitudes and an unsteadier baseline were observable. The measurement was then paused and $1 / 4$ of the initial narcotic dosage was applied via the intraperitoneal line. Experiments were carried on after EEG amplitudes were stable again.

Electroretinograms were recorded from the corneal surface of the left eye with a silicon-coated $\mathrm{Ag} / \mathrm{AgCl}$-wire. The silicon coat was removed from the part of the wire that was placed onto the cornea. The pupil of the left eye was dilated with one drop of $1 \%$ atropine sulfate. A needle reference electrode was inserted subcutaneously into the skin between the eyes and a needle ground electrode was inserted into the skin above the tail. Signal conductance was ensured by moistening the eye and the corneal electrode with regular electrode-gel. Initially, the eye and the $\mathrm{Ag} / \mathrm{AgCl}$-electrode with the gel were moistened with $0.9 \%$ saline regularly throughout the experiment. However, it turned out that the gel tends to dry and stick to cornea and conductant wire. Later experiments were performed with the same $\mathrm{Ag} / \mathrm{AgCl}$-electrode embedded into a $2 \%$ agarose-gel based on Ringers solution. This way, we were sure to avoid mechanical damage to the cornea and desiccation of the eye. The agarose-block was also moistened with a drop of $0.9 \%$ saline every 30 minutes. In control experiments with and without the agarose gel, we found that there was no significant influence on either amplitude, frequency or waveform of the ERG recordings (data not shown). These control experiments were performed with 10 C57/BL6 mice (5 with agarose block, 5 without agarose block). Later experiments not performed by the author were carried out with methylcellulose moistened with saline as a signal conducting agent. 
Full-field white light flashes were generated by 30 white LEDs placed circularly behind the mouse head inside a self-made "Ganzfeld" bowl. The generation of light flashes was controlled via the computer-assisted measurement station Tucker-Davis-Technologies (TDT, Ft. Lauderdale, FL, USA) System II (early experiments) and III (later and current experiments). Using the BioSig 32 and SigGen 1.3.1 software (provided by TDT) stimulus lengths, inter-stimulusintervals and stimulus attenuation were arranged
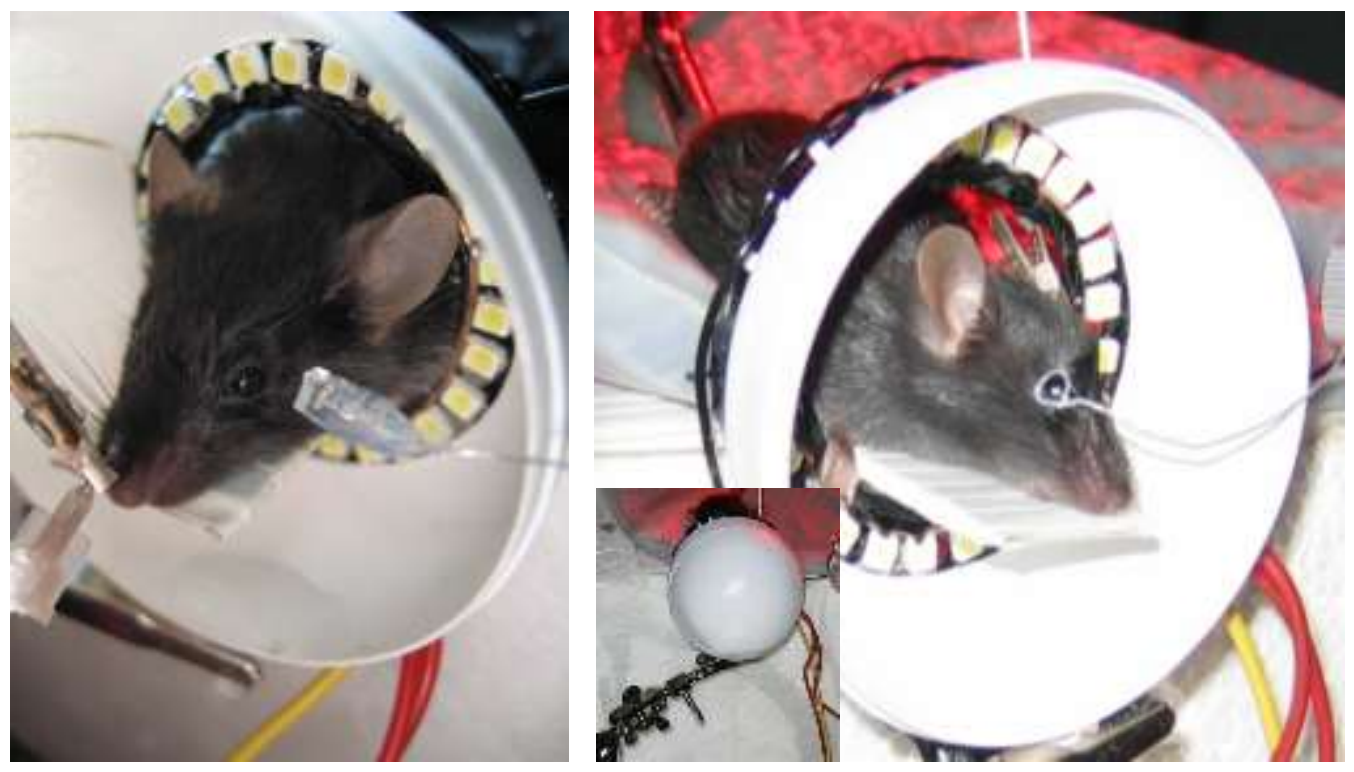

Figure 5: Anaesthetized test animals in ERG Ganzfeld setup

Left: The recording electrode embedded into a $2 \%$ agarose block based on Ringer's solution. The size of the agarose block is exaggerated here for demonstration reasons. Reference electrode in the subcutaneous tissue of the nose in situ. Right: Mouse head in the Ganzfeld bowl. Reference electrode on the nose removed here fo better overview. For demonstration reasons, the electrode ring wire's size is exaggerated and the right eye was used. Small picture: Ganzfeld bowl closed during ERG recordings. 


\subsubsection{Stimulus generation and data acquisition}

Preface: Parts of this section were adapted with the kind allowance of Ms. Dania Pauli-Magnus from her 2010 Göttingen M.D. thesis, who used the same hardware setup for her ABR and ASSR measurements and by: Biedermann, F. Universität Leipzig, Nat. Dissertation, 2004.

TDT II and III is a measurement station for real-time stimulus generation and acquisition of experiment data. The TDT System is based on an XBUS, controlling and synchronizing four modules. The modules can be controlled manually or via a computer-assisted array processor (AP2), which is connected to the XBUS via a light-guide cable. AP2 generates the test stimuli and controls the data transfer to the XBUS modules. The AP2 processor generates digital stimuli. Downstream modules to the AP2 are the following:

- A two-channel A/D-D/A converter module transforming digital into analogue stimuli and vice versa.

- FT6-2 (antialiasing filter), a programmable digital filter filtering interference out of the generated signal.

- After stimulus generation, light stimuli are lead to the programmable attenuator (PA5) modules, attenuating the maximum light intensity to the desired flash intensity. Attenuation was successively decreased from total darkness to maximum light intensity in 24 steps for scotopic and in 15 steps for photopic ERGs (see table 1).

- Via an amplification system (HeadBuffer System HB6), stimuli are lead to the custommade LED-ring in the experimental setting.

Flash intensities were calibrated (measurement of $\mathrm{cd} / \mathrm{s}^{2}$ emitted by the LED-ring) using two different calibration systems. Firstly, by the silicium photodiode Mavolux 5032c konstant and secondly by the IPL 10530 Integrated Photodiode Amplifier. Background illumination for light adaptation was at first performed by a flash light placed right in front of the Ganzfeld bowl with an intensity of $\sim 30 \mathrm{cds} / \mathrm{m}^{2}$. For avoidance of slack joint in the flash light, it was later replaced by an LED-Ring with six white LEDs applying a total light intensity of $\sim 30 \mathrm{cds} / \mathrm{m}^{2}$.

Electrical potentials were recorded at bandwidth (open filter $0,1-8000 \mathrm{~Hz}$ ) using a $50 \mathrm{~Hz}$ notch filter to eliminate interfering frequency feedback by the alternating $50 \mathrm{~Hz}$ line current and lead to the Amplifier (JHM Neuroamp 401). Amplification factor was 1000. Online averaging and storage were performed using BioSig $^{\circledR}$ Software. All measurements were performed in the same ex- 
perimental setting at the InnerEarLab of the Dept. of Otorhinolaryngology of the Göttingen University Medical Center.

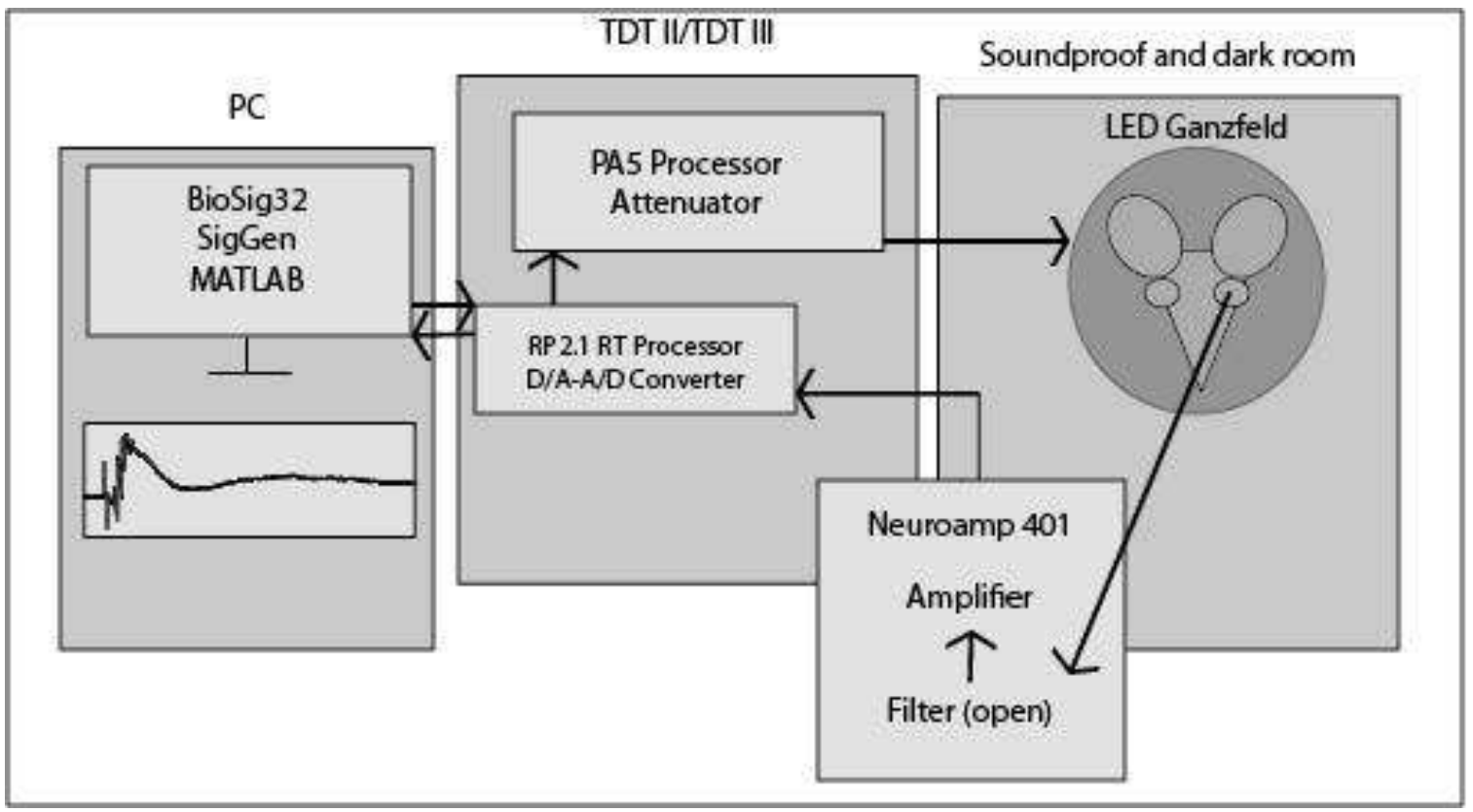

Figure 6: Simplified block diagram of the experimental setup

Different paradigms were developed and tested for the ERG recordings. Scotopic responsecurves were measured with white light flashes of three different stimulus-lengths $(0,1 \mathrm{~ms}, 1 \mathrm{~ms}$, $5 \mathrm{~ms}$ ). Prior to this study, only $0,1 \mathrm{~ms}$ stimuli were presented. To clarify, whether the stimulus length makes any difference in ERG amplitudes or maybe more clearly reveals the single ERG components, we also tested longer stimuli of $1 \mathrm{~ms}$ and $5 \mathrm{~ms}$. According to our previous protocol, inter stimulus intervals (ISIs) of 2 seconds were chosen for the first set of experiments. In following experiments, ISIs were changed to 5 seconds for light intensities below $1 \mathrm{cds} / \mathrm{m}^{2}$ and 17 seconds for light intensities above $1 \mathrm{cds} / \mathrm{m}^{2}$ (following the protocol by Jaissle et al. 2001). Recordings at a given stimulus duration were performed with increasing light intensity. In the beginning, the light flashes were strongly attenuated. The attenuation was decreased in 15 (photopic) or 24 (scotopic) steps up to full light intensity (see table 2). At each light intensity, responses were averaged ten times. The three stimulus lengths were performed successively starting with 
the scotopic recordings from the shortest stimulus duration to the longest. Then, the mouse was adapted to a constant background illumination of $30 \mathrm{cds} / \mathrm{m}^{2}$ for 10 minutes and photopic measurements followed, also running from the shortest stimulus duration to the longest. Total recording time was about 4 hours per animal. Not all animals were killed after the experiments. They were warmed and watched carefully on waking up after anasthesia and transferred back again to the Max-Planck-Institute for Experimental Medicine, Göttingen. Post-experimental preparation of the retinae for further morphological studies was not carried out by the author. If an animal was not used for preparation of the retina, it was killed after completion of the experiment by an intrapulmonary injection of 0,5 ml Embutramid (T61, Intervet GesmbH, Austria). 
Materials and Methods

Attenuator steps and corresponding light intensity of flashes for scotopic ERG measurements

\begin{tabular}{|r|r|r|r|r|}
\hline Point & 'Attenuator steps (scot) & 'cds/m_scot_0.1ms' & 'cds/m²_scot_1ms' & 'cds/m²_scot_5ms' \\
\hline 0 & 30 & 0.000167 & 0.00167 & 0.00835 \\
\hline 1 & 29.9 & 0.000239 & 0.00239 & 0.012 \\
\hline 2 & 29.8 & 0.000317 & 0.00317 & 0.0159 \\
\hline 3 & 29.7 & 0.000401 & 0.00401 & 0.02 \\
\hline 4 & 29.6 & 0.00049 & 0.0049 & 0.0245 \\
\hline 5 & 29.5 & 0.000585 & 0.00585 & 0.0292 \\
\hline 6 & 29.4 & 0.000686 & 0.00686 & 0.0343 \\
\hline 7 & 29.3 & 0.000792 & 0.00792 & 0.0396 \\
\hline 8 & 29.2 & 0.000904 & 0.00904 & 0.0452 \\
\hline 9 & 29.1 & 0.00102 & 0.0102 & 0.0511 \\
\hline 10 & 29 & 0.00115 & 0.0115 & 0.0573 \\
\hline 11 & 28 & 0.0027 & 0.027 & 0.135 \\
\hline 12 & 27 & 0.00482 & 0.0482 & 0.241 \\
\hline 13 & 26 & 0.00751 & 0.0751 & 0.375 \\
\hline 14 & 25 & 0.0108 & 0.108 & 0.539 \\
\hline 15 & 24 & 0.0146 & 0.146 & 0.73 \\
\hline 16 & 23 & 0.019 & 0.19 & 0.951 \\
\hline 17 & 22 & 0.024 & 0.24 & 1.2 \\
\hline 18 & 21 & 0.0295 & 0.295 & 1.48 \\
\hline 19 & 20 & 0.0357 & 0.357 & 1.78 \\
\hline 20 & 15 & 0.0748 & 0.748 & 3.74 \\
\hline 21 & 10 & 0.128 & 1.28 & 6.41 \\
\hline 22 & 5 & 0.196 & 1.96 & 9.8 \\
\hline 23 & 0.278 & 2.78 & 13.9 \\
\hline
\end{tabular}

Attenuator steps and corresponding light intensity of flashes for photopic ERG measurements

\begin{tabular}{|r|r|r|r|r|}
\hline Point & 'Attenuator steps (phot) & 'cds $/ \mathrm{m}^{2}$ phot_0.1ms' & 'cds $/ \mathrm{m}^{2} \_$phot_1 $\mathrm{ms}^{\prime}$ & 'cds/m_phot_5ms' \\
\hline 1 & 29 & 0.00115 & 0.0115 & 0.0573 \\
\hline 2 & 28 & 0.0027 & 0.027 & 0.135 \\
\hline 3 & 27 & 0.00482 & 0.0482 & 0.241 \\
\hline 4 & 26 & 0.00751 & 0.0751 & 0.375 \\
\hline 5 & 25 & 0.0108 & 0.108 & 0.539 \\
\hline 6 & 24 & 0.0146 & 0.146 & 0.73 \\
\hline 7 & 23 & 0.019 & 0.19 & 0.951 \\
\hline 8 & 22 & 0.024 & 0.24 & 1.2 \\
\hline 9 & 21 & 0.0295 & 0.295 & 1.48 \\
\hline 10 & 20 & 0.0357 & 0.357 & 1.78 \\
\hline 11 & 15 & 0.0748 & 0.748 & 3.74 \\
\hline 12 & 10 & 0.128 & 1.28 & 6.41 \\
\hline 13 & 5 & 0.196 & 1.96 & 9.8 \\
\hline 14 & 0 & 0.278 & 2.78 & 13.9 \\
\hline 15 & & & & \\
\hline
\end{tabular}

Table 1: Attenuation steps and resulting light intensities (in $\left.\mathrm{cds} / \mathrm{m}^{2}\right)$ at all used stimulus lengths $(0,1,1$ and 5ms) 


\subsection{Data Analysis}

In the beginning, single traces were manually analyzed online. Thresholds were defined at the first appearance of a clear ERG component (usually the b-wave). The threshold for the appearance of a- and b-wave at a certain attenuator step was detected visually by the experimenter (data not shown). Time zero was defined at the beginning of the stimulus, implicit times were estimated at the minimum of the a-wave and the maximum of the b-wave. The a-wave amplitude was taken from the baseline to the minimum of the a-wave. Absolute values for the a-wave amplitude were treated and presented as positive numbers. The b-wave amplitude was taken from the minimum of the a-wave to the maximum of the b-wave. If there was no detectable a-wave, the bwave amplitude was taken from the baseline to the maximum b-wave peak.

The raw data were analyzed by a customized MATLAB routine (MATLAB, The Mathworks, Natick, MA, USA). At this point, warmest thanks and highest appreciation must be directed to Mr. Dipl. Ing. phys. Gerhard Hoch, whose programming skills made the automized MATLAB analyses possible. All detected values were plotted into an overview Microsoft Excel® template designed by the author. Animals were separated into knockout $\left({ }^{--}\right)$and wildtype $\left({ }^{+/+}\right)$animals automatically. a-waves, b-waves and oscillatory potentials were shown in graphs plotted against their corresponding light intensity on a logarithmic scale. For all animals stimulus durations and all intensities, scotopic and photopic a- and b-wave amplitudes and latencies were demonstrated. For the oscillatory potentials, maximum amplitudes and maximum frequencies were demonstrated. Furthermore, one sheet for the stimulus intensities according to the protocol suggested by Roland Consult was created. All values (as the mean values of all measured animals) were plotted against their corresponding light intensity (in $\mathrm{cds} / \mathrm{m}^{2}$, see excursus) on a logarithmic scale.

The tables also showed mean values of all tested animals at all stimulus lengths. Standard errors of the means and t-tests were shown as well as graphics for comparison of all analyzed components showing knockout and wildtype animals next to each other. With this analyzing routine, results could be easily displayed and statistical significance or non-significance were revealed immediately.

Using another customized MATLAB routine, all single traces for each animal at each attenuator step were again analysed separately to demonstrate single animal examples (see single animal ERG examples in results section).

For this work, all diagrams were designed with Igor Pro® (WaveMetrics, Eugene, OR; USA). 
$\mathrm{Cd} / \mathrm{m}^{2}$ (Candela per square meter) is the SI unit for luminance. Candela $(\mathrm{Cd})$ is the SI unit for luminous intensity, square meters $\left(\mathrm{m}^{2}\right)$ is the unit for area (International SI-classification). Candelaseconds (Cds) is the measure of the light energy released by a single flash. One watt (W) equals one joule/second. A candela is $1 / 683$ watts. Thus a candela-second equals the energy $1 / 683$ joule (from: http://www.birket.com/technical-library/143/, downloaded on April, 15 $5^{\text {th }}, 2011$ ).

Excursus: Explanation of used SI units for description of light intensities 
One commercial ERG diagnostic system is provided by Roland Consult electrophysiological diagnostic systems. Later ERG experiments, performed by Mr. Christian Rüdiger, former technical assistant of the lab, were only performed for a representative selection of light intensities specified and used by Roland Consult electrophysiological diagnostic systems. The commercial system uses different stimulus lengths and intensities. We selected the light intensities of our protocol fitting the suggestions by Roland Consult electrophysiological diagnostic systems best, also independently of the stimulus length.

\begin{tabular}{|c|c|c|c|c|c|c|}
\hline \multicolumn{4}{|c|}{ Intensities of Flash programs } & \multicolumn{2}{|c|}{ 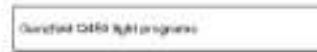 } & $\checkmark$ coNs \\
\hline & h & ind to the & ndard flas & $\propto d s / m^{2}=0$ & & \\
\hline \multirow{2}{*}{$\begin{array}{l}\text { flash- } \\
\text { length }\end{array}$} & \multicolumn{2}{|c|}{ Intensity of white flash } & \multicolumn{2}{|c|}{ Intensity of blue flash } & \multicolumn{2}{|c|}{ intensity of red fiash } \\
\hline & in cdsim ${ }^{2}$ & in $\mathrm{dB}$ & in cds $\mathrm{m}^{2}$ & In $\mathrm{dB}$ & in cds $\mathrm{m}^{2}$ & in $\mathrm{dB}$ \\
\hline $5 \mathrm{~ms}$ & $\overline{9,5}$ & +5 & 3,0 & 0 (standard) & 3,0 & 0 (standard) \\
\hline $1,58 \mathrm{~ms}$ & 3,0 & 0 (standard) & 0,95 & -5 & 0,95 & -5 \\
\hline $500 \mu 5$ & 0,95 & -5 & 0,3 & -10 & 0,3 & -10 \\
\hline $158 \mu \mathrm{s}$ & 0,3 & .10 & 0,095 & -15 & 0.095 & -15 \\
\hline $50 \mu \mathrm{s}$ & 0,095 & -15 & 0,03 & -20 & 0,03 & .20 \\
\hline $15,8 \mu \mathrm{s}$ & 0,03 & 20 & 0,0096 & 25 & 0,0095 & -25 \\
\hline $5 \mu \mathrm{s}$ & 0,0095 & -25 & 0,003 & -30 & 0,003 & -30 \\
\hline $5 \mu \mathrm{s}$ & 0,003 & -30 & 0,00095 & -35 & 0,00095 & -35 \\
\hline $5 \mu \mathrm{s}$ & 0,00095 & .35 & 0,0003 & .40 & 0,0003 & -40 \\
\hline $5 \mu \mathrm{s}$ & 0,0003 & .40 & 0,000095 & .45 & 0,000095 & .45 \\
\hline
\end{tabular}

Table 2: Flash intensity program of the Roland Consult Ganzfeld Q450 System for ERG experiments

\begin{tabular}{|r|r|r|r|r|}
\hline Point & 'InnerEarLab_cds $/ \mathrm{m}^{2 \prime}$ & 'RolandConsult_cds $/ \mathrm{m}^{2 \prime}$ & 'Flash Length (InnerEarLab)' & 'Flash Length (Roland Consult)' \\
\hline 0 & 0.0003 & 0.0003 & $0.1 \mathrm{~ms}$ & $5 \mu \mathrm{s}$ \\
\hline 1 & 0.0009 & 0.00095 & $0.1 \mathrm{~ms}$ & $5 \mu \mathrm{s}$ \\
\hline 2 & 0.0027 & 0.003 & $0.1 \mathrm{~ms}$ & $5 \mu \mathrm{s}$ \\
\hline 3 & 0.01 & 0.0095 & $0.1 \mathrm{~ms}$ & $5 \mu \mathrm{s}$ \\
\hline 4 & 0.03 & 0.03 & $0.1 \mathrm{~ms}$ & $15.8 \mu \mathrm{s}$ \\
\hline 5 & 0.075 & 0.095 & $0.1 \mathrm{~ms}$ & $50 \mu \mathrm{s}$ \\
\hline 6 & 0.28 & 0.3 & $0.1 \mathrm{~ms}$ & $158 \mu \mathrm{s}$ \\
\hline 7 & 0.75 & 0.95 & $1 \mathrm{~ms}$ & $500 \mu \mathrm{s}$ \\
\hline 8 & 2.8 & 3 & $1 \mathrm{~ms}$ & $1.58 \mathrm{~ms}$ \\
\hline 9 & 9.8 & 9.5 & $5 \mathrm{~ms}$ & $5 \mathrm{~ms}$ \\
\hline 10 & & & & \\
\hline
\end{tabular}

Table 3: Comparison of stimulus intensities used by the InnerEarLab and Roland Consult

Comparison of ERG stimulus light intensities used by a commercial ERG diagnostic system (Roland Consult electrophysiological diagnostic systems) and light intensities chosen out of our protocol being most alike. This comparison does not consider the different stimulus lengths used, just the resulting light intensity. 


\subsection{1 a-wave, b-wave, oscillatory potentials}

The original trace (Fig.7A) was $400 \mathrm{~Hz}$ lowpass filtered (Fig.7B). This way, the oscillatory potentials that lie between 90 and $130 \mathrm{~Hz}$ were not affected. The absolute minimum of this curve was detected and defined as the first negative peak of the oscillatory potentials. Going the trace backwards from the first negative peak defined as the first oscillation, the next local minimum was defined the a-wave. Then, the trace was smoothed with an 8-point-average window (Fig.7C) to calculate the absolute latency and amplitude of the a-wave. Although the a-wave is a negative peak, the absolute values were treated as positive numbers.

To detect the b-wave, the $400 \mathrm{~Hz}$ filtered trace was lowpass filtered at $20 \mathrm{~Hz}$ (Fig.7D). This way, the oscillatory potentials and the a-wave were smoothed. The maximum positive peak was defined the b-wave. The $20 \mathrm{~Hz}$ filtering was performed with an automatic correction of the phase shift to correctly define the latency.

The $400 \mathrm{~Hz}$ filtered trace was then $30 \mathrm{~Hz}$ high pass filtered (Fig.7E). This way, the oscillatory potentials became clearly visible. As the frequency of the oscillatory potentials tunes in and out, we defined the maximum amplitude as the representative wave for the frequency detection. 


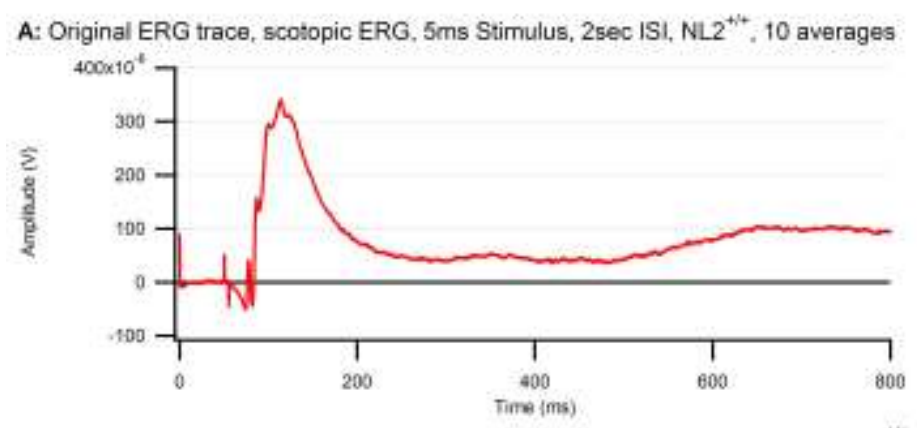

B: $400 \mathrm{~Hz}$ lowpass fitered ERG trace, scotopic ERG, $5 \mathrm{~ms}$ Stimulus, 2 sec ISI, NL2 ${ }^{\text {ti*; }}$, 10 averages

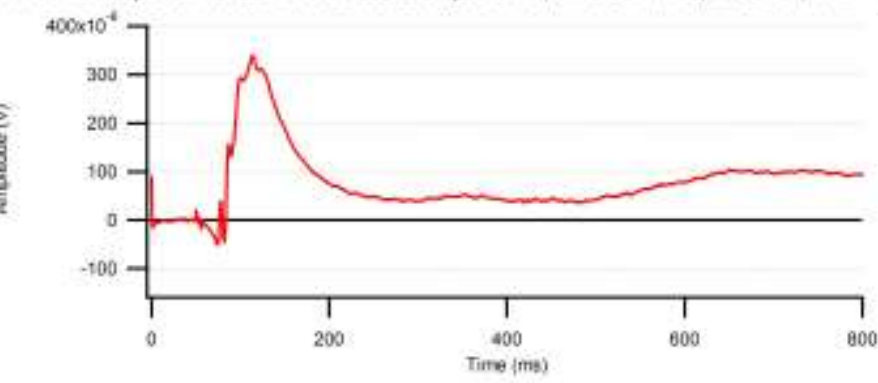

C: $400 \mathrm{~Hz}$ lowpass and 8pt average smoothed ERG trace, scotopic ERG, $5 \mathrm{~ms}$ Stimulus, 2sec ISI, NL2 $2^{4, t}, 10$ averages

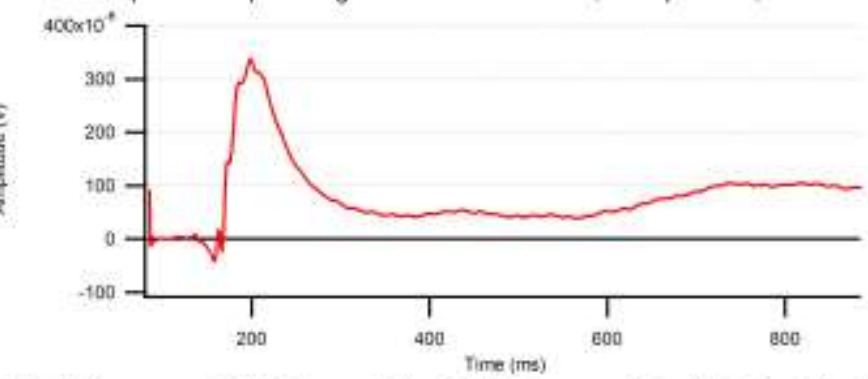

D: $400 \mathrm{~Hz}$ lowpass anf $20 \mathrm{~Hz}$ lowpass filtered ERG trace, scotopic ERG, $5 \mathrm{~ms}$ Stimulus, 2sec ISI, NL2, ${ }^{4+4} 10$ averages

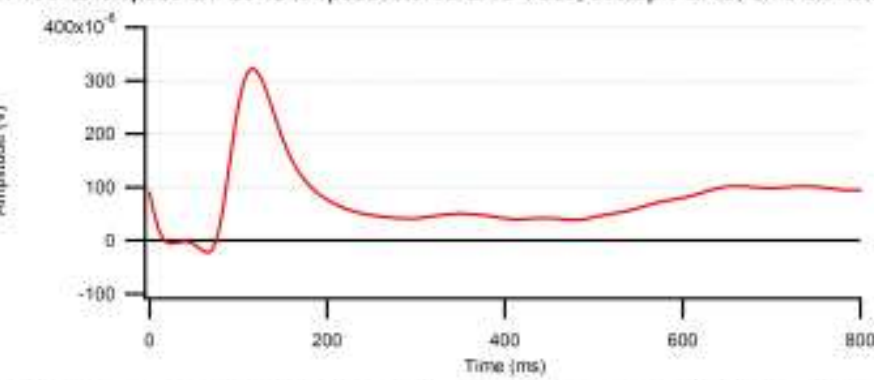

E: $400 \mathrm{~Hz}$ lowpass and $30 \mathrm{~Hz}$ highpass filtered ERG trace, scotopic ERG, $5 \mathrm{~ms}$ Stimulus, 2sec ISI, NL2 $2^{\text {tit }}, 10$ averages $150 \times 10^{1}$

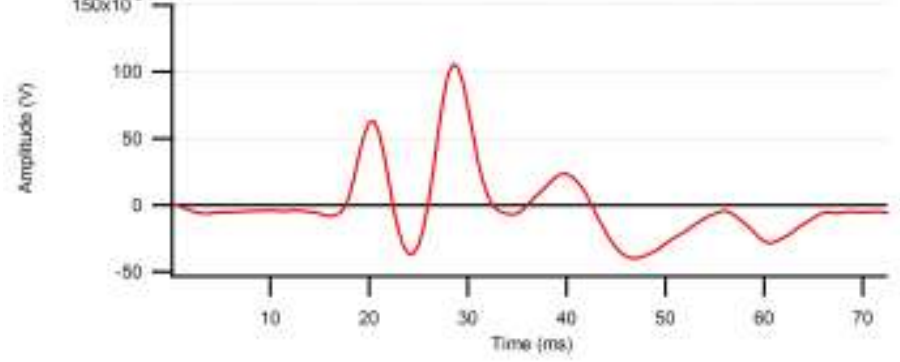

Figure 7: Filtered traces for detection of ERG components

Example of an unfiltered ERG trace (10 averages) (A), 400Hz filtered ERG trace (10 averages) (B), ERG trace smoothened with an $8 \mathrm{pt}$ moving average (10 averages) (C), $20 \mathrm{~Hz}$ filtered ERG trace (10 averages) (D), $30 \mathrm{~Hz}$ highpass filtered ERG trace (E). 


\subsubsection{OP frequency detection}

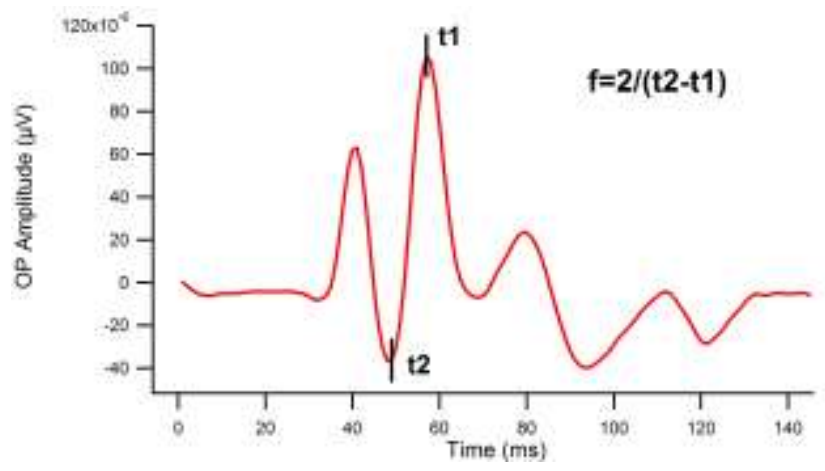

\section{Figure 8: OP frequency detection}

$\mathrm{f}$ (in Hertz Hz) is defined 1/s. Oscillation period/interval is $\mathrm{T}$ (in $\mathrm{ms}$ ), so $\mathrm{f}=1 / \mathrm{T}$. In our analysis, $\mathrm{T}$ was defined by subtracting $\mathrm{ms}$ at $\mathrm{t} 1$ from the $\mathrm{ms}$ at the next local minimum at $\mathrm{t} 2$, treated as absolute number. This interval equals half the oscillation. We calculate $\mathrm{f}=2 /(\mathrm{t} 1-\mathrm{t} 2)$. So our frequency is $\mathrm{f}=1 / \mathrm{T} / 2$. To calculate the correct (whole) oscillations' frequency, the formula needs to be dissolved to $\mathrm{f}={ }^{2} /(\mathrm{T} / 2)$ 

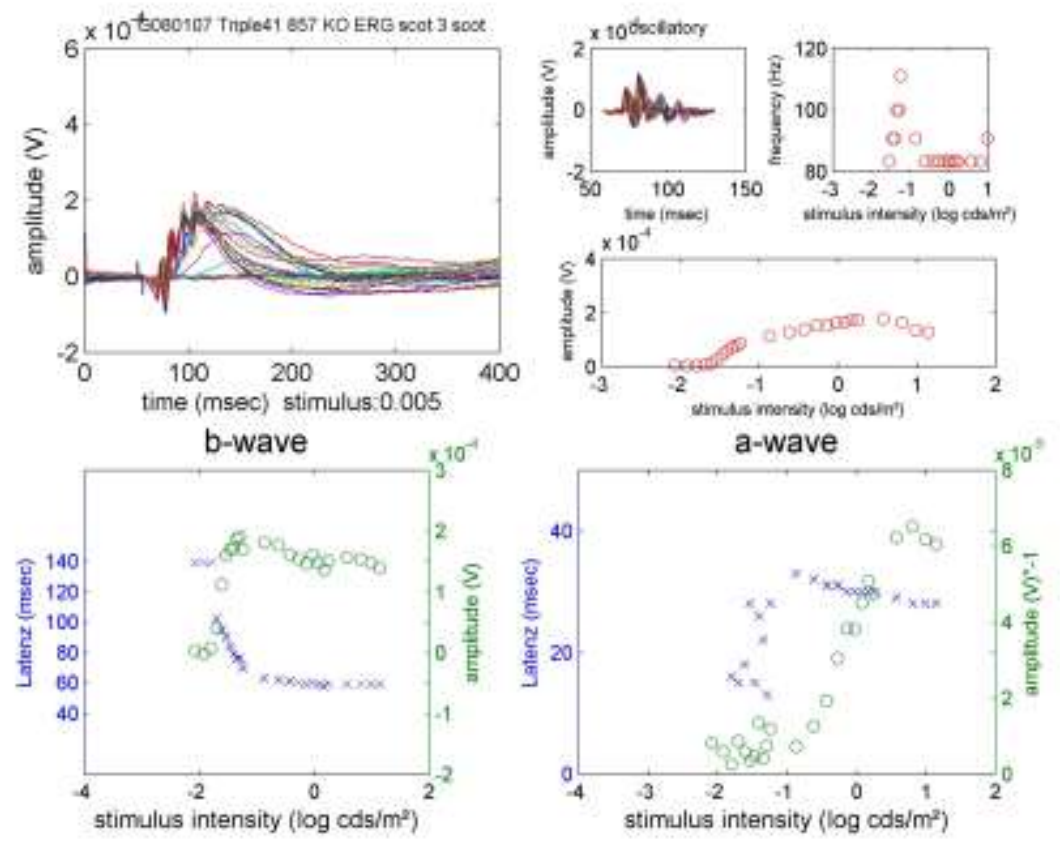

Figure 9: Overview illustration of data analysis for each animals' ERG measurement

Example chosen here is a Protein 4.1 Triple knockout animal a stimulus length of $5 \mathrm{~ms}$, ISI of $5 \mathrm{sec}$ and $17 \mathrm{sec}$. Left upper corner shows all traces at all attenuation levels. Left lower corner shows amplitudes and latencies of $b$-waves, right lower corner shows amplitudes and latencies of a-waves and right upper corner shows filtered OPs', frequencies and amplitudes of OPs.

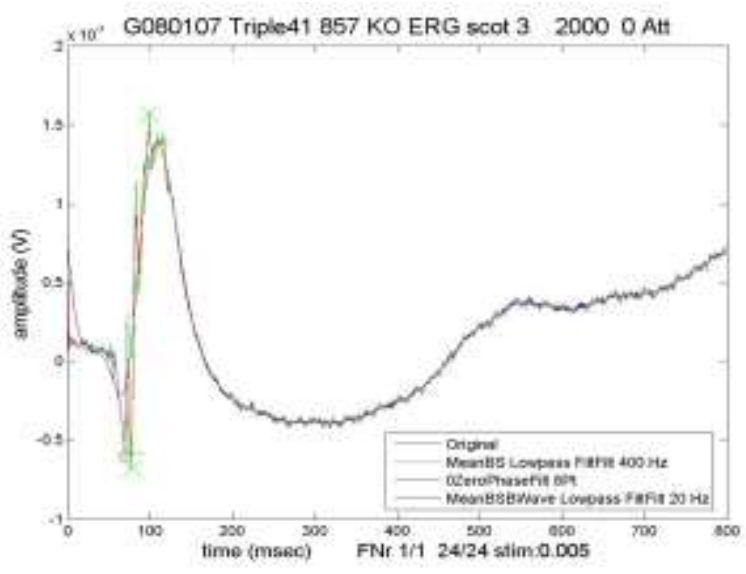

Figure 10: Summary plot of filtered ERG traces

Fig.9 and 10 show how the original ERG traces are analyzed one by one by our customized MatLab Routine. Each ERG run of each animal is analyzed in the same way and identical overview illustrations were created for each animal. 


\subsection{Auditory Brainstem Response - ABR}

Electric Response Audiometry (ERA) is an objective hearing test that registers specific changes in the EEG evoked by acoustic stimuli. Next to the possibility of identifying the hearing threshold objectively, its main value is the topic localization of hearing disorders within the sensory hearing pathway. The test used on our animals investigates the so-called early acoustic evoked potentials (EAEP). They occur within $10 \mathrm{~ms}$ after the acoustic stimulus and reflect electric activity from the cochlea, the hearing nerve and the brainstem. EAEPs can either be obtained via electrocochleography or the ABR (auditory brainstem response). We focus here on the ABR. Electrocochleograms were not obtained from our test animals. The ABR consists of characteristic waves (named Jewett(J) I-V(VII)), their number varying between five and seven. Each wave can be attributed to different structures along the hearing pathways from primary perception to processing in deeper brain structures and, finally, the hearing cortex.

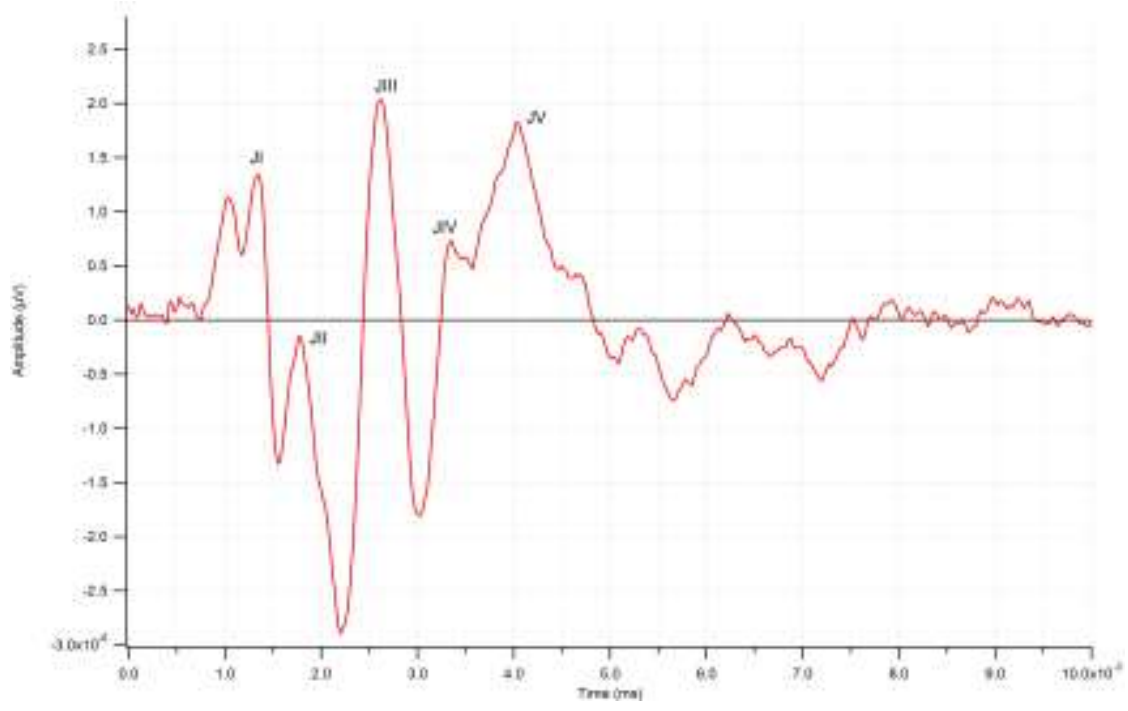

Figure 11: ABR Example; 20Hz click ABR at 80dB $\left(\mathrm{NL}^{+/+}\right)$

Wave I (JI) is located to the cochlear spiral ganglion cells. The spiral ganglion cells depend on stimulation by the primary cells of acoustic perception, which would be the cochlear inner and outer hair cells. Wave II (JII) is located to the cells of the nucleus cochlearis. Wave III (JIII) originates in the nucleus cochlearis and from the contralateral nucleus olivaris superior. Wave IV (JIV) originates from ipsi- and contralateral nucleus olivaris superior and anteroventral nucleus of nucleus cochlearis. Wave V (JV) is attributed to the anterolateral nucleus of the nucleus cochlearis, the lateral lemniscm and the inferior collicle (Steward, 2000). Wave JVI and JVII only inconstantly appear in the ABR and are not taken into consideration in our analysis. 


\section{Results}

\subsection{Electroretinograms}

Mean amplitudes of a-wave and b-wave (in $\mu \mathrm{V}$ ) were plotted against their corresponding light intensity on a logarithmic scale $\left(\log \mathrm{cds} / \mathrm{m}^{2}\right)$. Mean values of the waves' latencies (in ms) were plotted against their corresponding time course (in ms). For the oscillatory potentials, mean values of amplitudes (in $\mu \mathrm{V}$ ) and OP frequency (in $\mathrm{Hz}$ ) were plotted against their corresponding light intensity on a logarithmic scale $\left(\log \mathrm{cds} / \mathrm{m}^{2}\right)$. Single plots were created for all stimulus lengths $(0,1 \mathrm{~ms}, 1 \mathrm{~ms}$ and $5 \mathrm{~ms})$.

In one plot, results were shown only for a selection of light intensities (independently of the stimulus length). These light intensities were chosen accordingly to the protocol of Roland Consult electrophysiological diagnostic systems, who offer a commercial Ganzfeld flash ERG recording system (Ganzfeld Q450). Not all acquired data will be shown in the results section.

\subsection{Auditory brainstem response}

The results of auditory physiology measurements of NL2- and Protein $4.1^{+/+}$and ${ }^{-/-}$mice will also be shown.

Hearing thresholds (in $\mathrm{dB}$ ) at specific clear tones (in $\mathrm{kHz}$ ) were acquired.

Thresholds for the appearance of the ABR waves JI-JV will be shown. Amplitudes (in $\mu \mathrm{V}$ ) and latencies (in ms) of the threshold waves JI to JV are plotted against their corresponding sound pressure level (in $\mathrm{dB})$.

No statistically significant alterations in the KO animals were observed, neither in the NL2 $2^{-/-}$, nor in the 4.1Triple ${ }^{-/}$. 


\subsection{Neuroligin 2}

Three sets of ERG measurements for the $\mathrm{NL}^{-/-}$animals and their $\mathrm{NL}^{+/+}$littermates were performed. The first series of experiments was carried out by the author using ISIs of 2 seconds throughout. These experiments showed a significant alteration of the $\mathrm{NL}^{-/-}$animals compared to their wild-type littermates ( $n=9$ for $\mathrm{NL}_{2}^{-/-}$and $n=9$ for $\mathrm{NL}^{+/+}$). In the second and third series of experiments, performed by Mr. Christian Rüdiger (former lab technician supervised by myself and Prof. Moser), we aimed to confirm the alterations observed. The second experimental series was performed using a different ISI in accordance with other working groups in retinal physiology. 5 seconds were used for stimuli smaller than $1 \mathrm{cds} / \mathrm{m}^{2}$ and 17 seconds for stimuli from $1 \mathrm{cds} / \mathrm{m}^{2}$ on. This set of experiments showed very small absolute amplitudes and no significant alteration between $\mathrm{NL}^{-/-}$and $\mathrm{NL}_{2}^{+/+}$animals $\left(\mathrm{n}=4\right.$ for $\mathrm{NL}^{-/-}$and $\mathrm{n}=5$ for $\mathrm{NL}^{+/+}$). The third and last set of experiments were performed using the longer ISIs and stimulus intensities according to the protocol suggested by Roland Consult electrophysiological diagnostic systems. In this protocol, only 10 light intensities at different stimulus lengths were tested compared to 3 times 24 (72) light intensities. This set of experiments revealed the largest absolute amplitudes of all measurements and did not reveal statistically significant differences between $\mathrm{NL}_{2}^{-{ }^{-}}$and NL2 $2^{+/+}$ animals ( $\mathrm{n}=5$ for $\mathrm{NL} 2^{-/-}$and $\mathrm{n}=4$ for $\mathrm{NL} 2^{+/+}$).

The absolute amplitudes observed in the second set of experiments were much lower than in the first and third set. The system was checked several times and experimental settings were compared and analysed. A technical reason/error for the much lower amplitudes could not be found out but also not excluded completely. These data were eventually published, because they were reproducible in the third set of measurements, while the statistically significant difference between $\mathrm{NL}_{2}^{+/+}$and $\mathrm{NL}^{-/-}$animals found in the first set of experiments performed with the 2 second ISI could not be reproduced. The third set of experiments showed again no statistically significant difference between $\mathrm{NL}_{2}^{-/-}$and $\mathrm{NL}_{2}^{+/+}$, even though absolute amplitudes were large. All three sets of experiments will be shown here. The results of the second experimental set were published by Hoon et al. in 2009 .

Photopic measurements were only carried out during the first series of experiments using short ISIs of 2 seconds and did not show statistically significant differences between NL2 ${ }^{-/-}$and $\mathrm{NL} 2^{+/+}$. 


\subsubsection{Scotopic ERG measurements - Examples}

\subsubsection{First set of experiments}

A
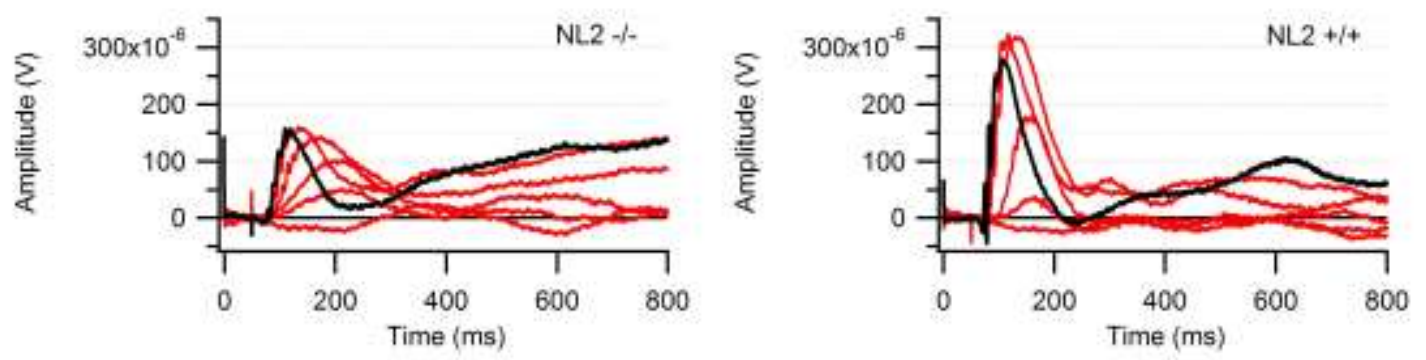

B
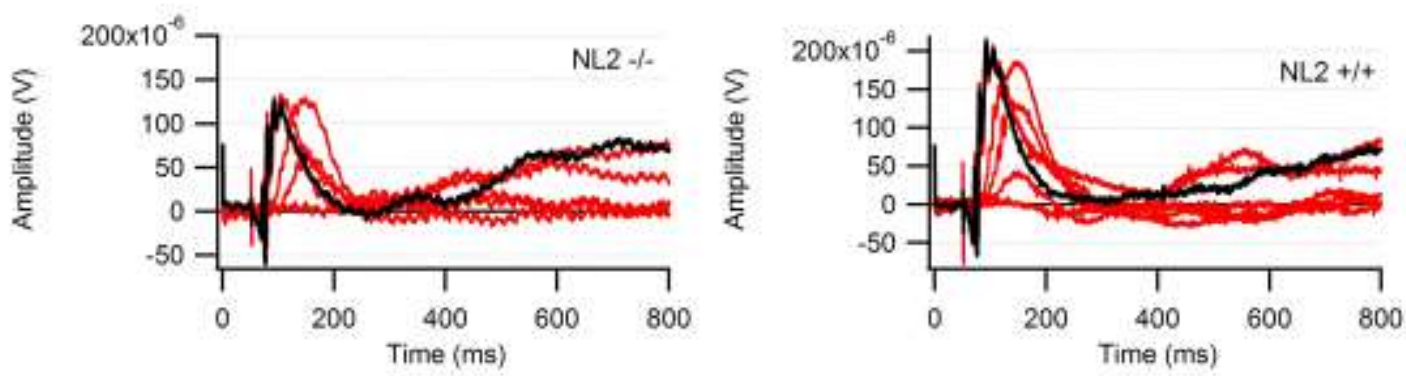

C
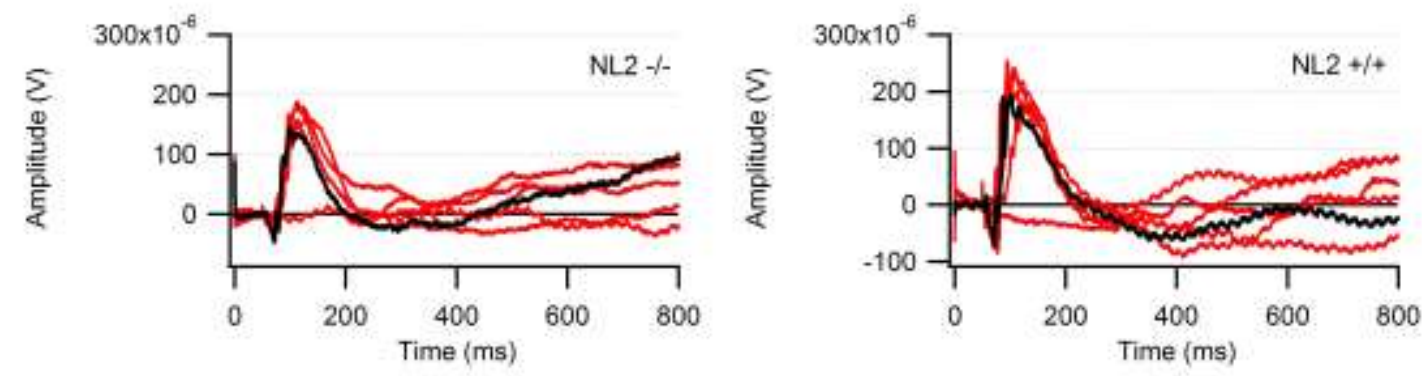

Figure 12: ERG examples of randomly chosen animals from the $1^{\text {st }}$ set of experiments $\left(\mathrm{NL}^{-/-}\right.$and $\left.\mathrm{NL2}^{+/+}\right)$.

$\bullet=\mathrm{NL2}^{+/+}, \bullet=\mathrm{NL2}^{-/-}$

Examples of ERG waves of randomly chosen animals growing with increasing light intensity. NL2 ${ }^{-/}$(left figures) and $\mathrm{NL}^{+/+}$(right figures). $\mathrm{NL}^{-/-}$traces' amplitudes at all measured stimulus lengths are reduced in amplitude.

A: 0,1 ms stimulus, 2sec ISI, 10 averages per trace; B: $1 \mathrm{~ms}$ stimulus, $2 \mathrm{sec}$ ISI, 10 averages per trace; C: $5 \mathrm{~ms}$ stimulus, 2sec ISI, 10 averages per trace; red lines show ERG traces at different attenuator steps, the black line is always the trace at highest light intensity (attenuator step 0 ). The flat red lines closest to the zero lines not showing observable ERG waves show subthreshold recordings in strongest flash attenuation. 


\subsubsection{Second set of experiments}

A
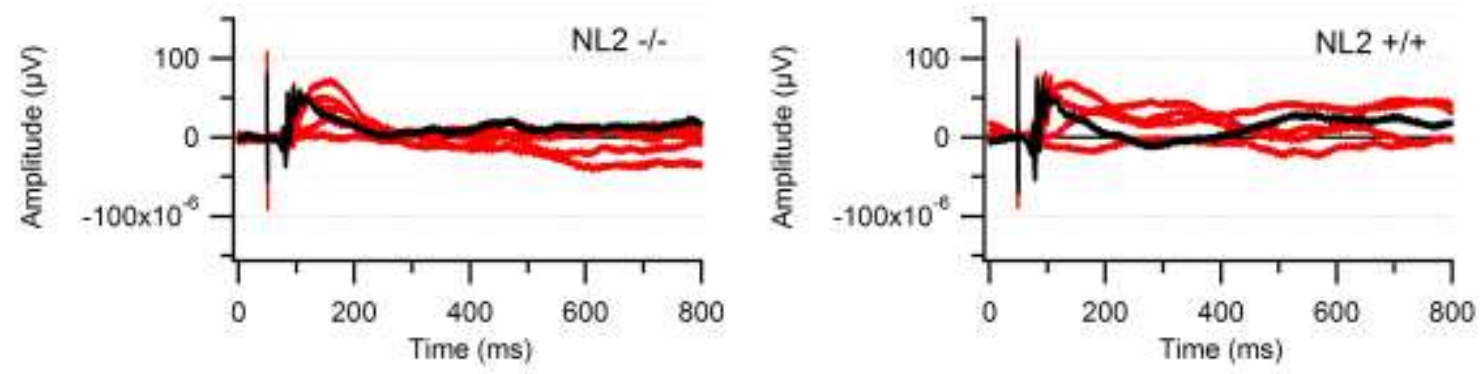

B
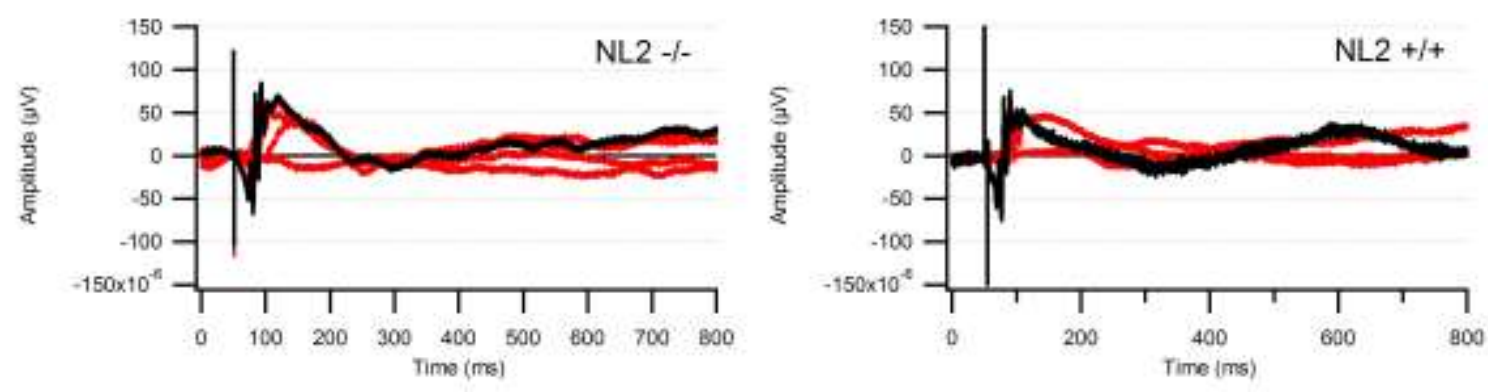

C
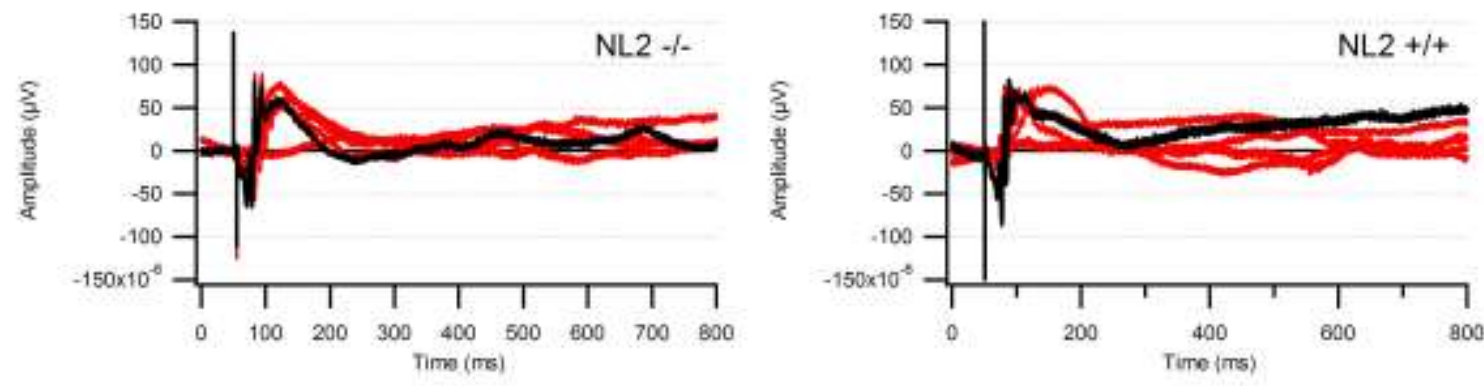

Figure 13: ERG examples of randomly chosen animals from the $2^{\text {nd }}$ set of experiments $\left(\mathrm{NL2}^{-/-}\right.$and $\mathrm{NL2}^{+/+}$). $\bullet=\mathrm{NL2}^{+/+}, \bullet=\mathrm{NL2}^{-/-}$

Examples of ERG waves of randomly chosen animals growing with increasing light intensity. NL2 ${ }^{-/}$(left figures) and $\mathrm{NL}^{+/+}$(right figures).

A: $0,1 \mathrm{~ms}$ stimulus, $5 / 17 \mathrm{sec}$ ISI, 10 averages per trace; B: $1 \mathrm{~ms}$ stimulus, $5 / 17 \mathrm{sec}$ ISI, 10 averages per trace; C: $5 \mathrm{~ms}$ stimulus, 5/17 sec ISI, 10 averages per trace; red lines show ERG traces at different attenuator steps, the black line is always the trace at highest light intensity (attenuator step 0 ). The flat red lines closest to the zero lines not showing observable ERG waves show subthreshold recordings in strongest flash attenuation. 


\subsubsection{Third set of experiments}
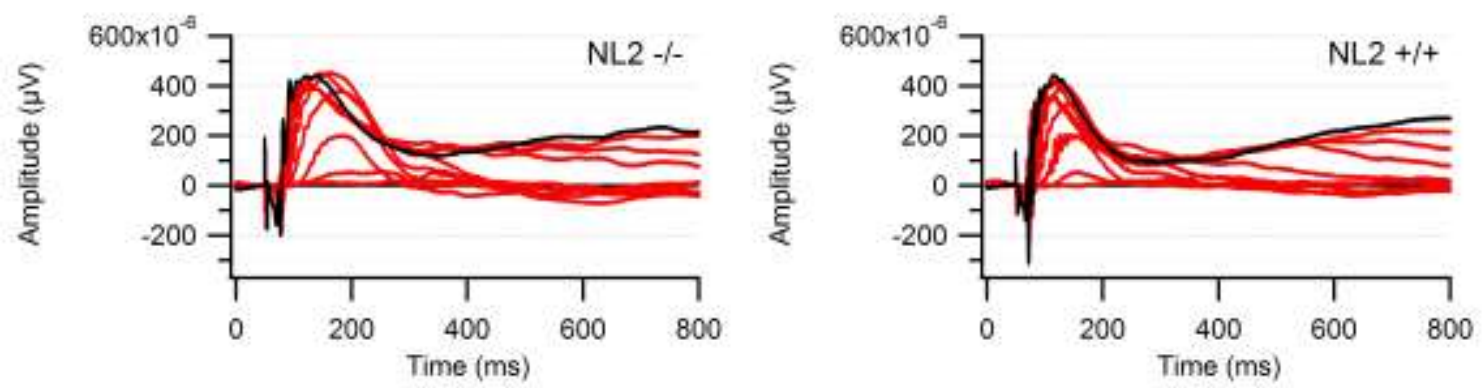

Figure 14: ERG examples of randomly chosen animals from the $3^{\text {rd }}$ set of experiments $\left(\mathrm{NL}^{-/}\right.$and $\left.\mathrm{NL}^{+/+}\right)$. $\bullet=\mathrm{NL2}^{+/+}, \bullet=\mathrm{NL2}^{-/}$

Example of ERG waves of randomly chosen animals growing with increasing light intensity. NL2 ${ }^{-/}$(left figures) and $\mathrm{NL}^{+/+}$(right figures).

A: Stimulus intensities according to Roland Consult ERG protocol, 5/17sec ISI, 10 averages per trace; red lines show ERG traces at different attenuator steps, the black line is always the trace at highest light intensity (attenuator step 0 ). The flat red lines closest to the zero lines not showing observable ERG waves show subthreshold recordings in strongest flash attenuation.

Fig.12-14 show examples of ERG recordings of randomly chosen $\mathrm{NL}^{+/+}$and $\mathrm{NL}^{-/-}$mice. The black line shows the ERG trace at maximum light intensity (Attenuation 0), the red lines are ERG traces at higher attenuation levels to demonstrate growth of amplitudes and shortening of latencies with increasing light intensity. Maximum light intensity, as shown by the black lines, does not always cause maximum amplitudes. Fig.12A-C show examples taken from the first set of experiments. On direct comparison of a randomly chosen $\mathrm{NL}^{-/-}$(left) and $\mathrm{NL}^{+/+}$(right) mouse at all measured stimulus lengths, the $\mathrm{NL}^{-/-}$animal shows smaller amplitudes in all acquired ERG traces. Fig.13A-C show examples taken from the second set of experiments with longer ISIs. Direct comparison of a randomly chosen $\mathrm{NL}^{-/-}$(left) and $\mathrm{NL}^{+/+}$(right) mouse at all measured stimulus lengths reveals no statistically significant altered amplitudes between knockout and wildtype animal in all acquired ERG traces. It is important here to note the very small absolute amplitudes in both knockout and wildtype animal. Fig.14 shows examples taken from the third set of experiments. A randomly chosen $\mathrm{NL}^{-/-}$(left) and $\mathrm{NL}^{+/+}$(right) mouse measured according to the Roland Consult paradigm is presented. This paradigm does not consider different stimulus lengths, but only considers the resulting light intensity, which is why only one panel is shown here. Knockout and wildtype animal do not present with significantly altered amplitudes in all acquired ERG traces, even though absolute amplitudes are even higher than in the first and second experimental set. 


\subsubsection{Amplitudes}

\subsubsection{First set of experiments}

A

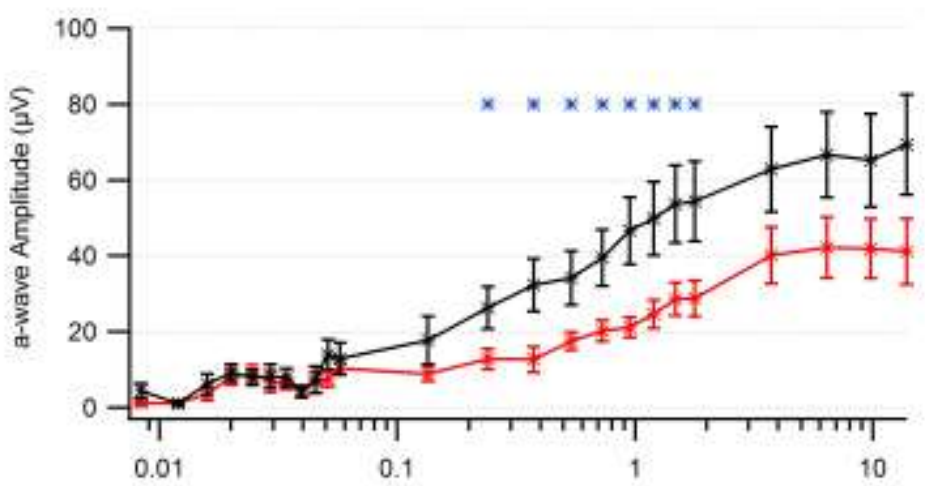

B

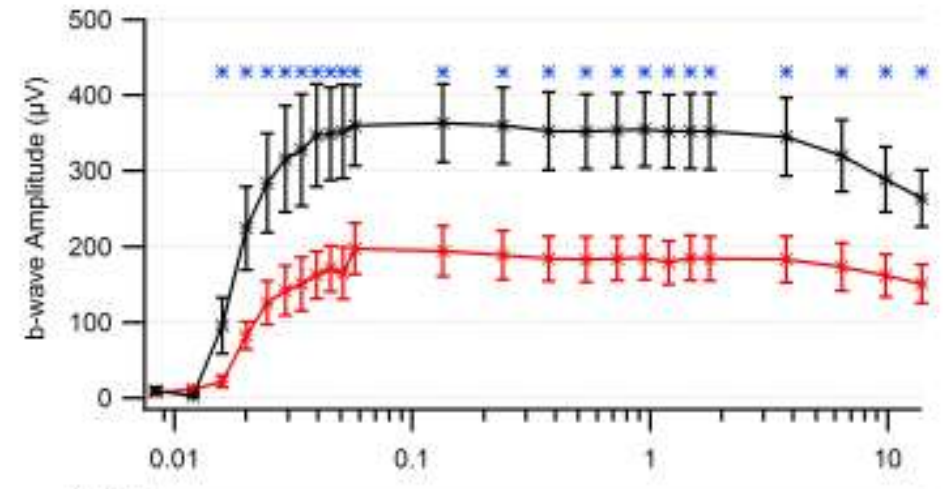

C

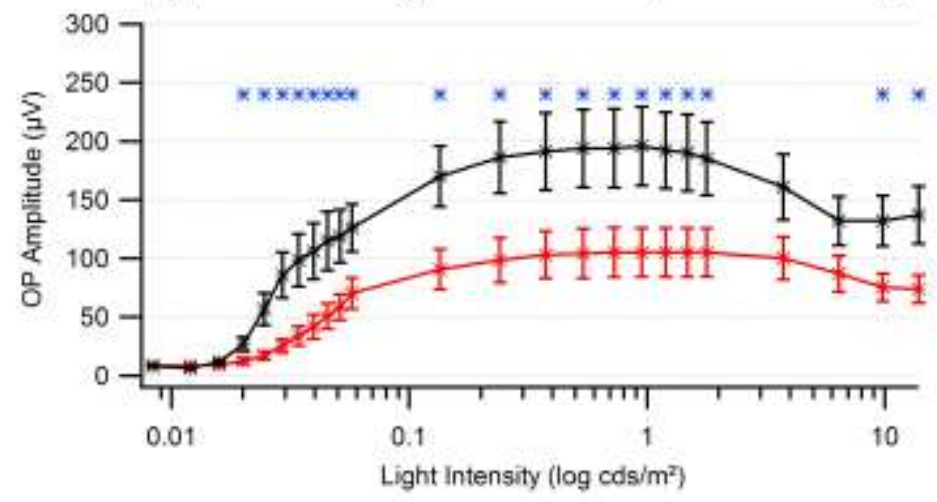

Figure 15: Amplitudes of a-waves, b-waves and OPs from the $1^{\text {st }}$ set of experiments $\left(\mathrm{NL2}^{-/}\right.$and $\left.\mathrm{NL2}^{+/+}\right)$.

$*=\mathrm{p} \leq 0,05, *=\mathrm{NL2}^{+/+}, *=\mathrm{NL}^{2 /-}$

Amplitudes (in $\mu \mathrm{V}$ ) of a-waves (A), b-waves (B) and oscillatory potentials (C) of NL2 ${ }^{-/}$mice ( $\mathrm{n}=8$, red lines) in comparison with their $\mathrm{NL}^{+/+}$littermates ( $\mathrm{n}=9$, black lines). Results shown here from the first set of experiments performed with $5 \mathrm{~ms}$ white flash light stimulus and $2 \mathrm{sec}$ ISI. Plotting against increasing light intensity in logarithmic scaling. Statistically significant reduction of amplitudes in KO animals was found for all three ERG components. 


\subsubsection{Second set of experiments}

A

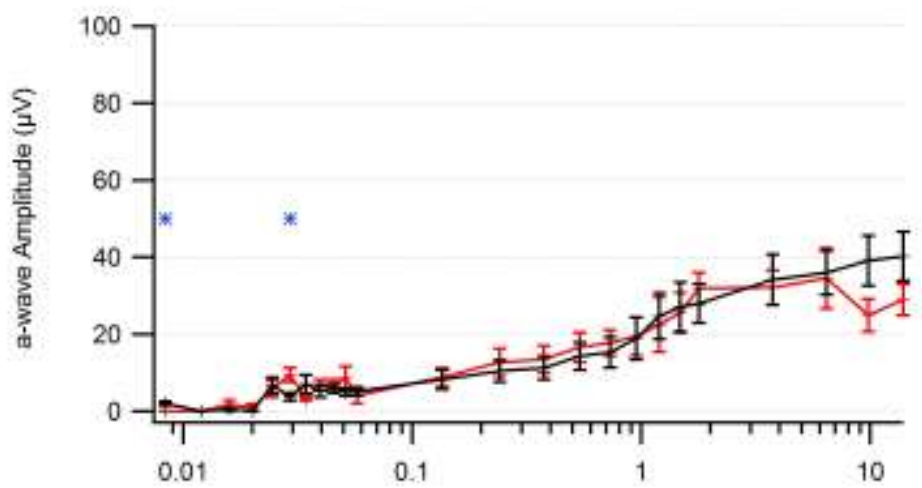

B

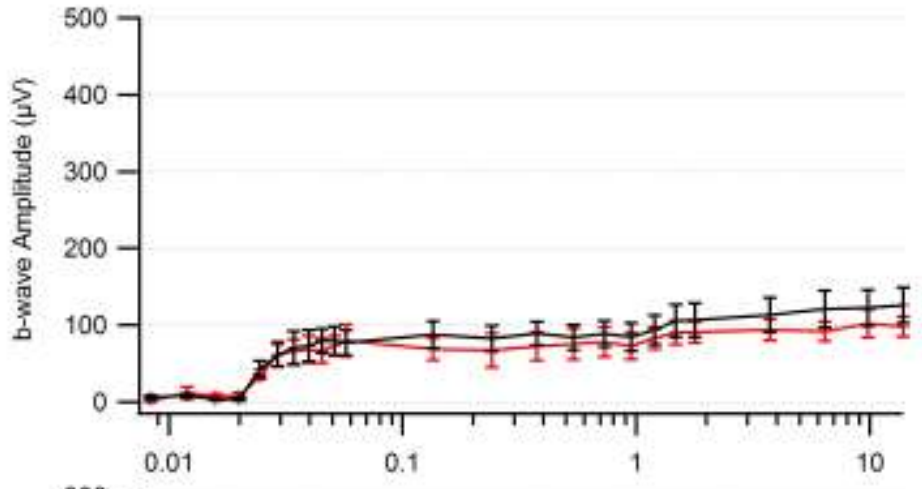

C

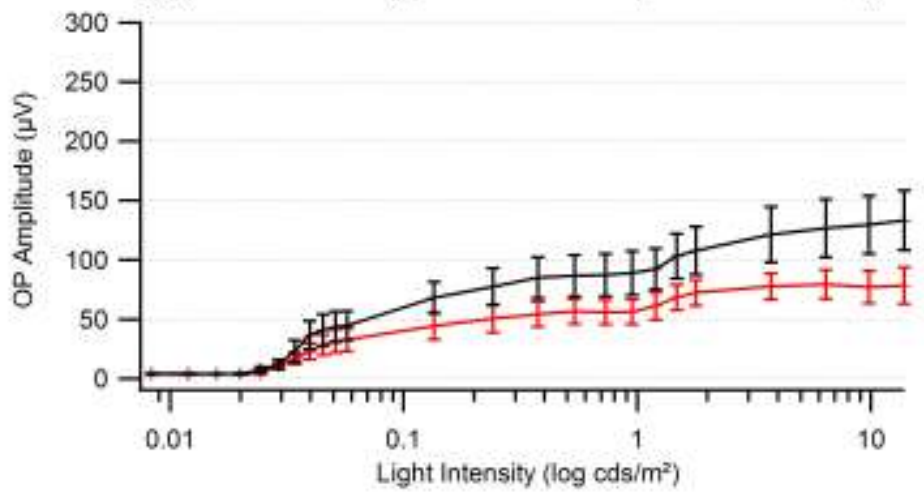

Figure 16: Amplitudes of a-waves, b-waves and OPs from the $2^{\text {nd }}$ set of experiments $\left(\mathrm{NL}^{-/}\right.$and $\left.\mathrm{NL2}^{+/+}\right)$.

$*=\mathrm{p} \leq 0,05, *=\mathrm{NL2}^{+/+}, *=\mathrm{NL2}^{-/}$

Amplitudes (in $\mu \mathrm{V}$ ) of a-waves (A), b-waves (B) and oscillatory potentials (C) of NL2 ${ }^{-/-}$mice ( $\mathrm{n}=4$ ) in comparison with their NL2 $2^{+/+}$littermates $(\mathrm{n}=5)$. Results shown here from the second set of experiments performed with a $5 \mathrm{~ms}$ white flash light stimulus, $5 \mathrm{sec}$ ISI for stimuli below $1 \mathrm{cds} / \mathrm{m}^{2}$ and $17 \mathrm{sec}$ for stimuli from $1 \mathrm{cds} / \mathrm{m}^{2}$. Plotting against increasing light intensity in logarithmic scaling. Significance is not maintained compared to the measurements shown above with a $2 \sec$ ISI $*=p \leq 0,05$. Overall amplitudes are significantly smaller than in the first set of experiments shown above. 


\subsubsection{Third set of experiments}

A

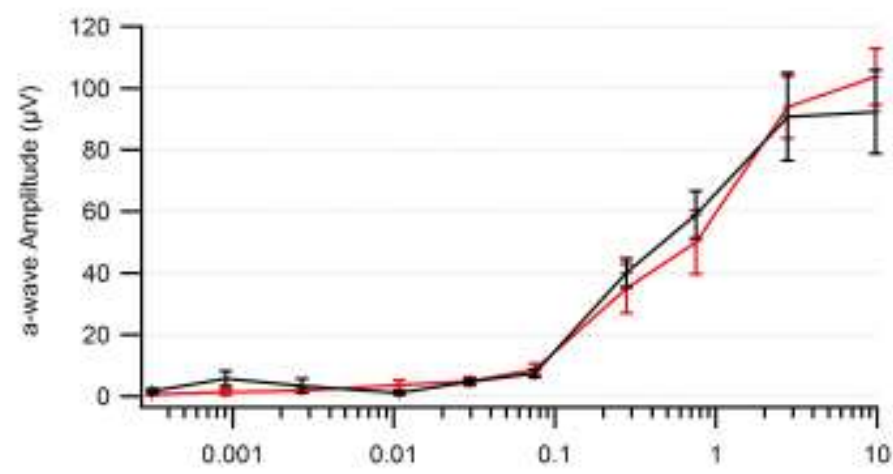

B

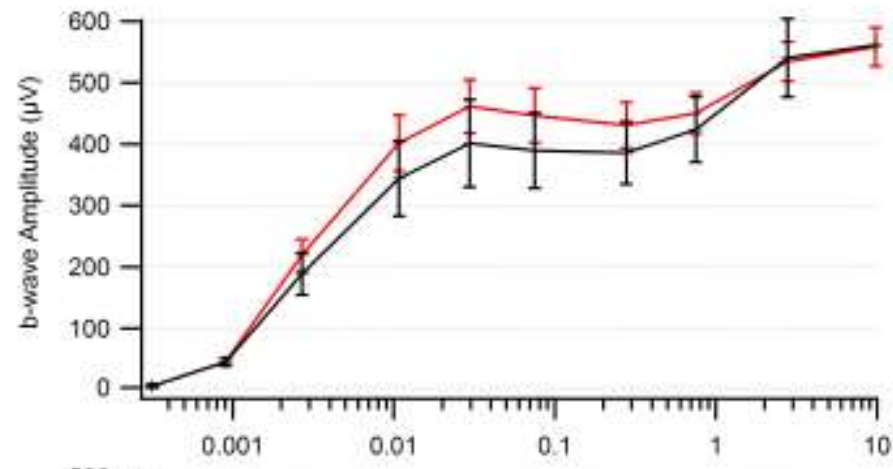

C

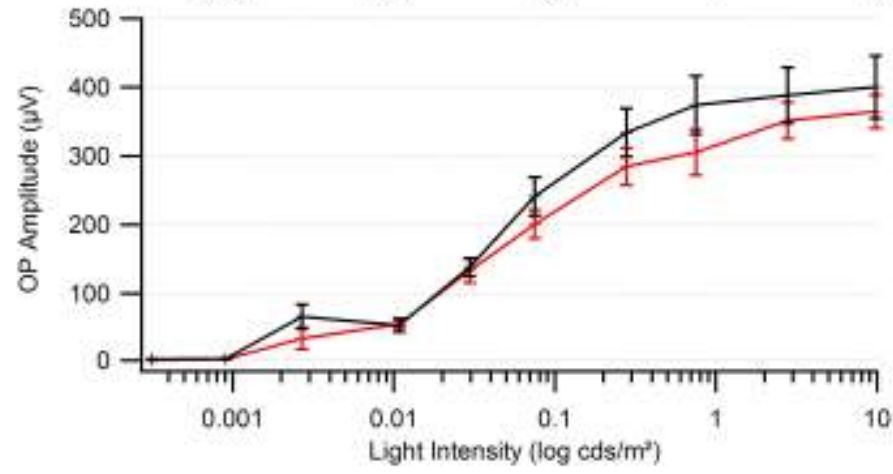

Figure 17: Amplitudes of a-waves, b-waves and OPs from the $3^{\text {rd }}$ set of experiments (NL2 ${ }^{-/-}$and NL2 $^{+/+}$).

$*=\mathbf{p} \leq \mathbf{0 , 0 5}, *=\mathrm{NL2}^{+/+}, *=\mathrm{NL} 2^{-/-}$

Amplitudes (in $\mu \mathrm{V}$ ) of a-waves (A), b-waves (B) and oscillatory potentials (C) of NL2 ${ }^{-/-}$mice ( $\mathrm{n}=5$ ) in comparison with their $\mathrm{NL}_{2}^{+/+}$littermates $(\mathrm{n}=4)$. Results shown here from the third set of experiments performed with white flash light stimuli of different stimulus lengths, $5 \mathrm{sec}$ ISI for light intensities below $1 \mathrm{cds} / \mathrm{m}^{2}$ and $17 \mathrm{sec}$ for light intensities from $1 \mathrm{cds} / \mathrm{m}^{2}$. Plotting against increasing light intensity in logarithmic scaling. Significance is not maintained, which is contradictory to the first set and consistent with the second set of results. Overall amplitudes are higher than in the first and second set of experiments shown above.

In the ERG recordings performed with an ISI of 2 seconds (first set of experiments, Fig.15), the $\mathrm{NL}^{-/-}$mice revealed smaller amplitudes of a-waves, b-waves and OPs in comparison with the corresponding $\mathrm{NL}^{+/+}$littermate controls. The a-waves showed a steady rise in amplitude with increasing light intensity at all stimulus lengths (data with $0,1 \mathrm{~ms}$ and $1 \mathrm{~ms}$ stimulus not shown 
here). The clearest responses were evoked at the maximum stimulus length of $5 \mathrm{~ms}$ (Fig.15A). Consistent reduction of a-wave amplitudes was observed in the $\mathrm{NL} 2^{-/-}$animals for all suprathreshold stimulus intensities. Statistically significant reduction in amplitude was observed at medium light intensities. The second set of experiments (Fig.16) showed consistently smaller absolute amplitudes for $\mathrm{NL}^{-/-}$and $\mathrm{NL}_{2}^{+/+}$animals, respectively. No statistically significant difference in a-wave amplitudes was observed with longer ISIs (Fig.16A). In the third set (Fig.17), absolute amplitudes were higher than in both earlier performed sets. Still, no significant reduction in a-wave amplitudes (Fig.17A) could be reproduced.

In the first set of experiments (Fig.15), the b-wave amplitudes rose steadily from lowest light intensities on. Good responses were recorded at all stimulus lengths. The NL2/- animals presented with statistically significant reduced amplitudes at all stimulus lengths and light intensities (Fig.15B). At medium light intensities, a plateau was observed at all stimulus lengths and a decline, more prominent in the $\mathrm{NL}_{2}^{+/+}$animals, but also indicated in the $\mathrm{NL}^{-{ }^{--}}$animals, was seen at maximum light intensities. The second set of experiments (Fig.16) showed consistently smaller absolute b-wave amplitudes for both $\mathrm{NL}^{-/-}$and $\mathrm{NL}^{+/+}$animals (Fig.16B). No statistically significant difference was observed between the $\mathrm{NL}^{-/-}$and $\mathrm{NL}^{+/+}$animals. In the third set of experiments (Fig.17), absolute amplitudes were high, a plateau at medium light intensities was observed, but no decline with further increasing light intensity ways seen. No statistically significant difference between NL2 ${ }^{+/+}$and $\mathrm{NL}^{-/-}$b-wave amplitudes could be reproduced (Fig.17B).

In the first set of experiments (Fig.15), good oscillatory potentials were recorded at all stimulus lengths (Fig.15C). From threshold on, amplitudes rose steadily, reaching a plateau and their maximum amplitude at medium light intensities (consistent with Algvere and Wachtmeister 1972, Wachtmeister 1973a, Wachtmeister and Hahn 1987). The NL2 ${ }^{-/-}$animals presented with statistically significant reduced OP amplitudes at all stimulus lengths and light intensities (Fig.15C). At medium light intensities, a plateau was observed at all stimulus lengths and a decline, more prominent in the $\mathrm{NL} 2^{+/+}$animals, was seen at maximum light intensities. The second set of experiments (Fig.16C) showed consistently smaller absolute OP amplitudes for NL2 ${ }^{+/+}$and NL2 ${ }^{-/-}$ animals, respectively. Eventhough the $\mathrm{NL}^{-/-}$animals presented a tendency towards lower amplitudes, this difference did not reach statistical significance. In the third set of experiments (Fig.17C), absolute OP amplitudes were higher than in both other data sets. No plateau at medium light intensities and no decline at highest stimuli intensities was observed. No statistically significant difference between $\mathrm{NL}^{-/-}$and $\mathrm{NL}^{+/+}$b-wave amplitudes could be reproduced. 


\subsubsection{Temporal components}

\subsubsection{First set of experiments}

A

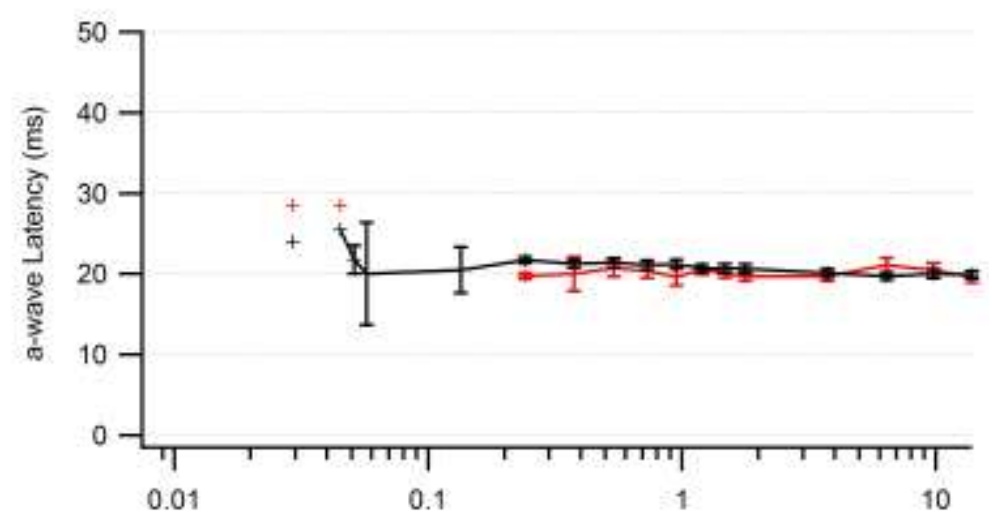

B

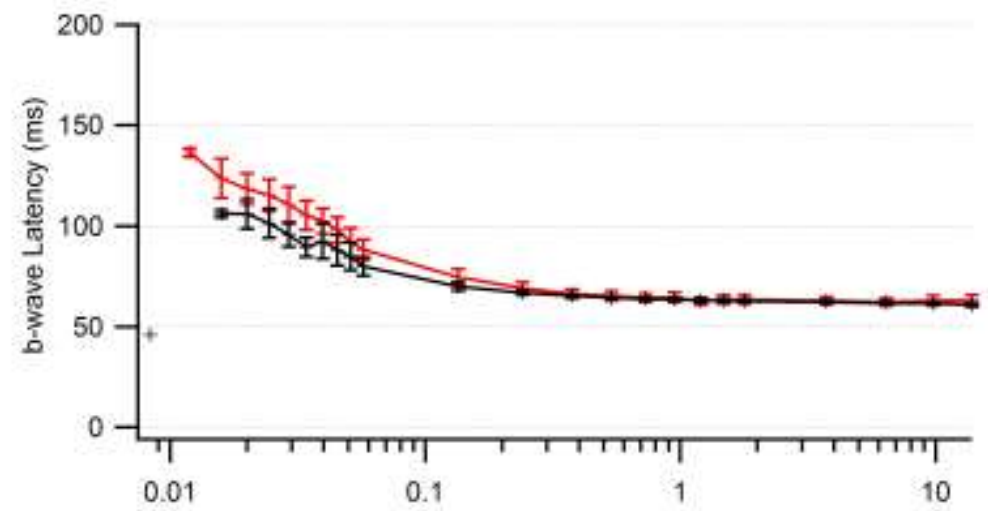

C

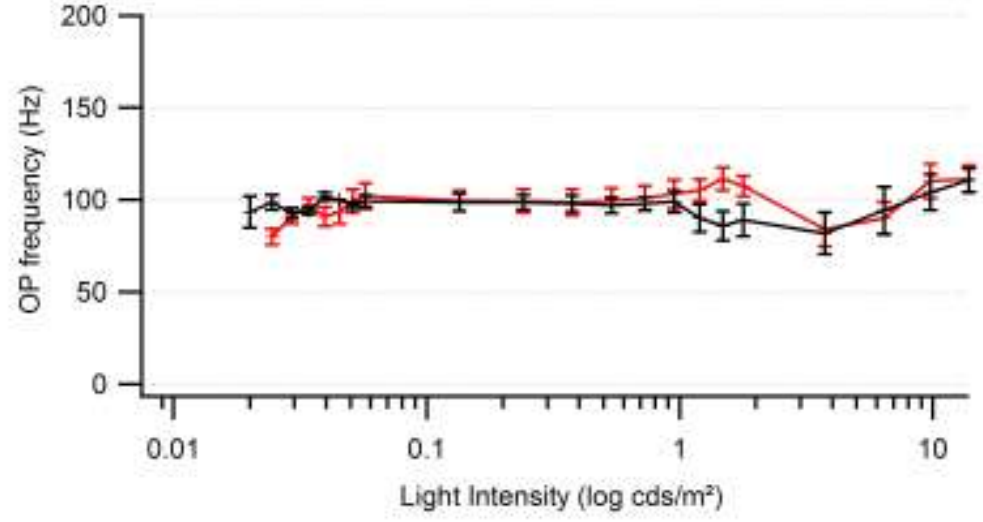

Figure 18: Latencies of a-waves, b-waves and frequencies of OPs from the $1^{\text {st }}$ set of experiments $\left(\mathrm{NL2}^{-/ /}\right.$and $\left.\mathrm{NL2}^{+/+}\right)$.

$*=\mathrm{NL2}^{+/+}, *=\mathrm{NL}^{-/-}$

Latencies (in ms) of a-waves (A), b-waves (B) and frequency (in $\mathrm{Hz}$ ) of oscillatory potentials (C) of NL2 ${ }^{-/}$mice $(n=8)$ in comparison with their NL2 $2^{+/+}$-littermates $(n=9)$. Plotting against increasing light intensity in logarithmic scaling. Measurements shown here for $5 \mathrm{~ms}$ white flash light stimulus with $2 \mathrm{sec}$ ISI. No significant differences in either latencies or frequencies were obtained. 


\subsubsection{Second set of experiments}

A

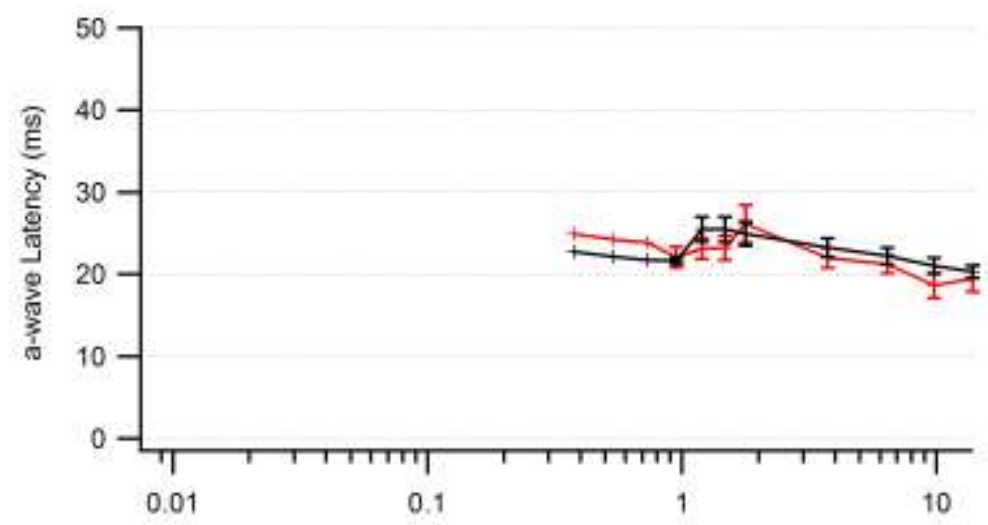

B

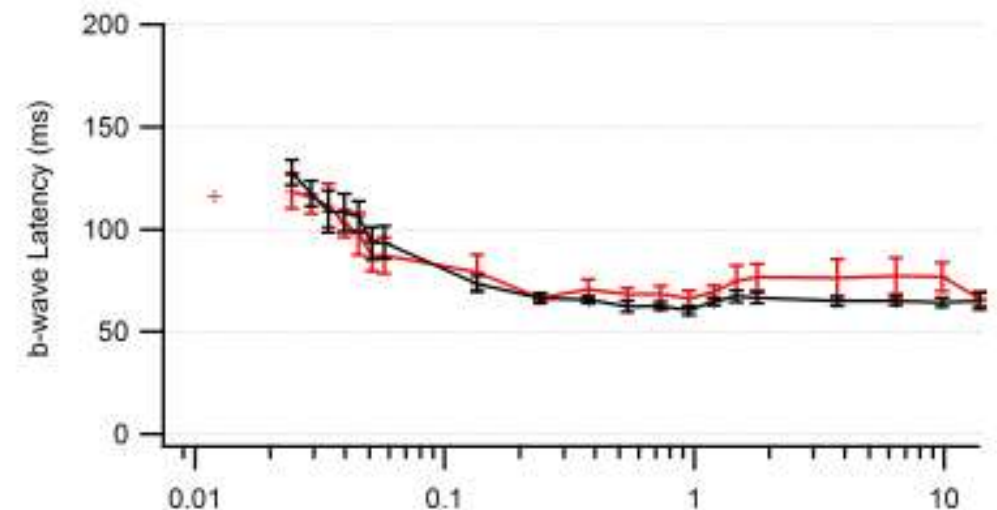

C

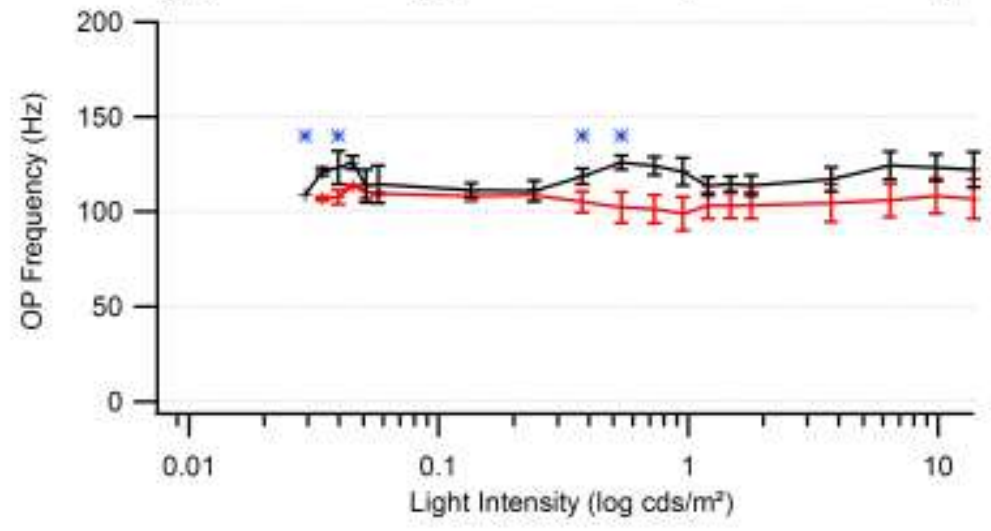

Figure 19: Latencies of a-waves, b-waves and frequencies of OPs from the $2^{\text {nd }}$ set of experiments (NL2 $2^{-/-}$and $\left.\mathrm{NL}^{+/+}\right)$.

$*=\mathbf{p} \leq \mathbf{0 , 0 5}, *=\mathrm{NL}^{+/+}, *=\mathrm{NL} 2^{-/-}$

Latencies (in ms) of a-waves (A), b-waves (B) and frequency of oscillatory potentials (C) of NL2 $2^{-/-}$mice $(n=4)$ in comparison with their NL2 ${ }^{+/+}$-littermates $(n=5)$. Measurements shown here for $5 \mathrm{~ms}$ white flash light stimulus with 5 sec ISI for light intensities below $1 \mathrm{cds} / \mathrm{m}^{2}$ and $17 \mathrm{sec}$ for light intensities above $1 \mathrm{cds} / \mathrm{m}^{2}$. Plotting against increasing light intensity in logarithmic scaling. Results are in accordance with the results of measurements with 2 sec ISI shown above with no consistent statistical significance $*=\mathrm{p} \leq 0,05$. 


\subsubsection{Third set of experiments}

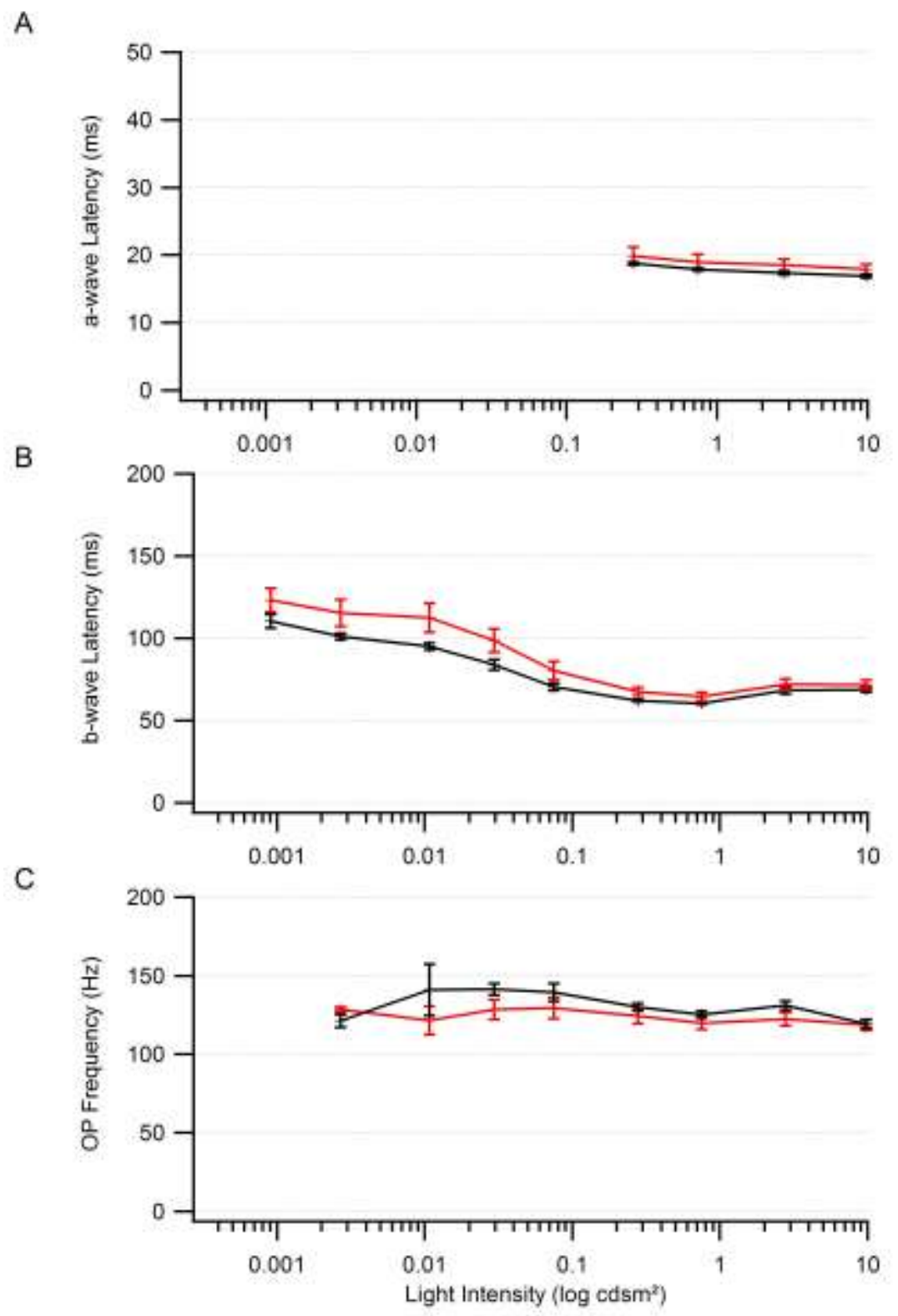

Figure 20: Latencies of a-waves, b-waves and frequencies of OPs from the $3^{\text {rd }}$ set of experiments (NL2 ${ }^{-/}$and $\left.\mathrm{NL2}^{+/+}\right)$.

$*=\mathrm{NL2}^{+/+}, *=\mathrm{NL2}^{-/}$

Latencies (in ms) of a-waves (A), b-waves (B) and frequency of oscillatory potentials (C) of NL2 ${ }^{-/}$mice $(n=5)$ in comparison with their $\mathrm{NL}^{+/+}$-littermates $(\mathrm{n}=4)$. Measurements shown here for $5 \mathrm{~ms}$ white flash light stimulus with 5 sec ISI for light intensities below $1 \mathrm{cds} / \mathrm{m}^{2}$ and $17 \mathrm{sec}$ for light intensities above $1 \mathrm{cds} / \mathrm{m}^{2}$. Plotting against increasing light intensity in logarithmic scaling. Results are in accordance with the results of the first and second experimental set, showing no statistically significant differences between $\mathrm{NL}_{2}^{+/+}$and $\mathrm{NL} 2^{-/-}$. 
The a-waves showed constant latencies in all three sets of experiments. No statistically significant difference was seen between $\mathrm{NL}^{-/-}$and $\mathrm{NL}^{+/+}$a-wave latencies (Fig.18A, 19A and 20A). The b-wave latencies (Fig.18B, 19B and 20B) declined with increasing light intensities. The NL /- animals did not present with statistically significant alterations in the latencies of the b-waves. Furthermore, in no set of our experiments did the $\mathrm{NL}^{-/-}$animals present with statistically significant alterations in their OP frequencies (Fig.18C, 19C and 20C). OP frequency was very stable over the range of light intensities at all stimulus lengths. The measured OP frequency lay between $80-120 \mathrm{~Hz}$. Wachtmeister in his $1973 \mathrm{~b}$ article stated that the dominant frequency of the OPs decreases from about $150 \mathrm{~Hz}$ in a relatively more dark-adapted condition to about $105 \mathrm{~Hz}$ in a more light adapted state and as the ISI is shortened. Therefore, our data are in the range of previous observations. It seems that neither the ISI nor the deletion of NL2 has a significant effect on the temporal properties of the ERG components. 


\subsubsection{Photopic ERG measurements}

A

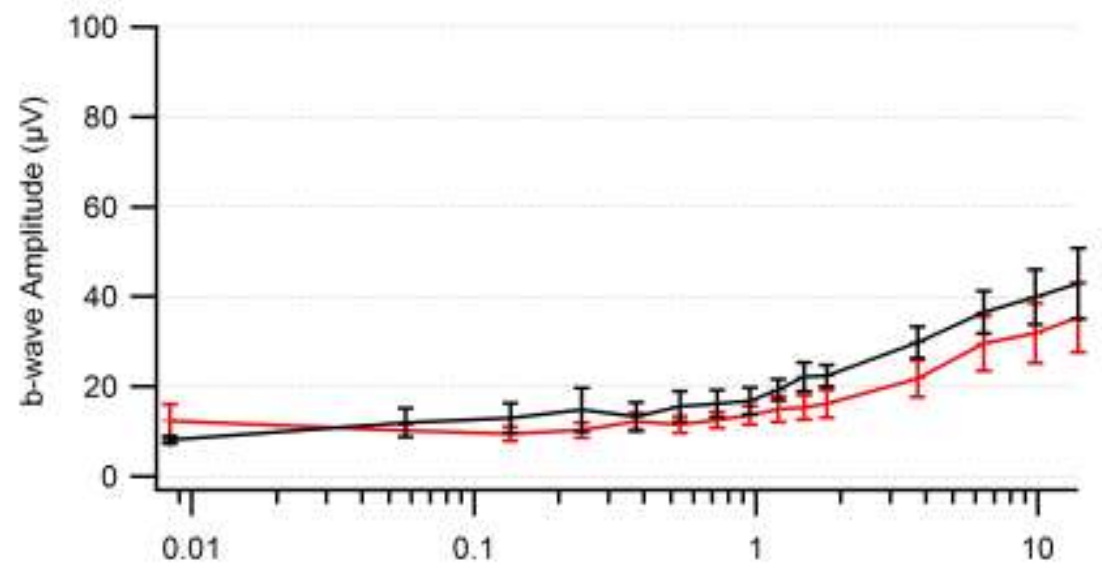

B

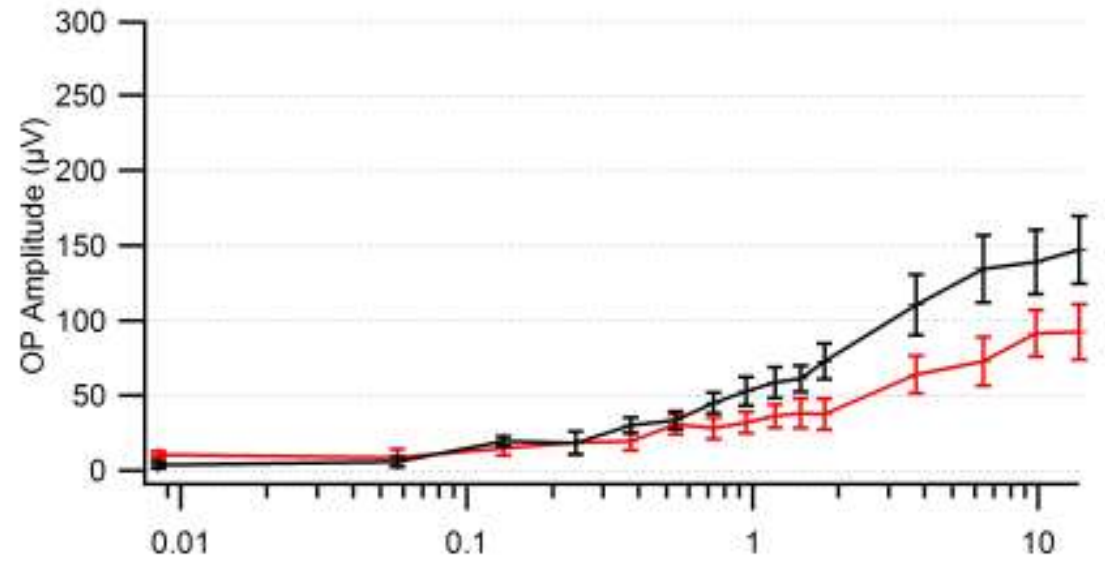

C

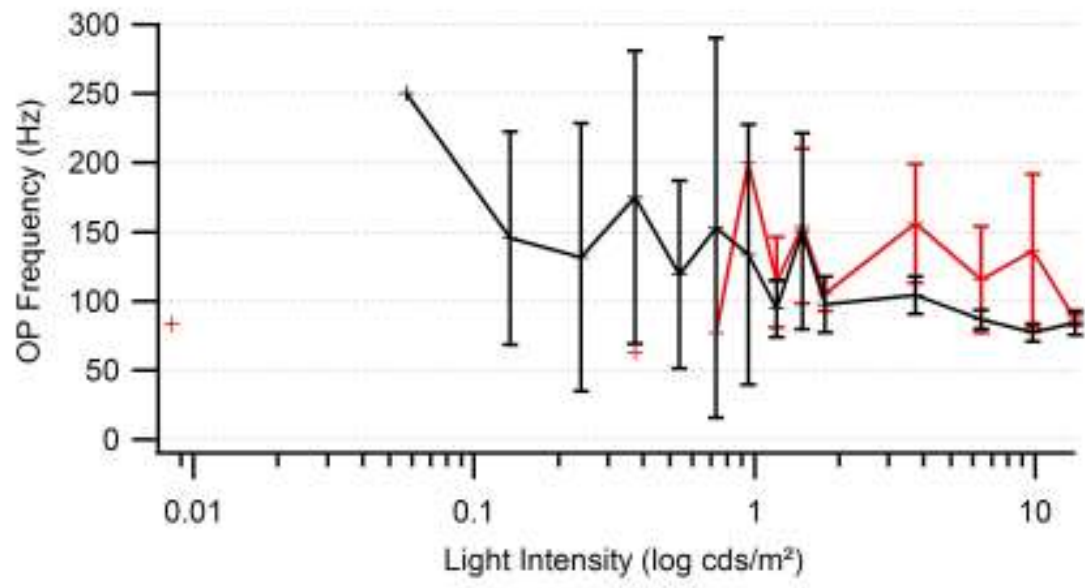

Figure 21: Photopic b-wave and OP amplitudes and OP frequencies from $1^{\text {st }}$ set of experiments $\left(\mathrm{NL}^{-/}\right.$and $\left.\mathrm{NL2}^{+/+}\right)$.

$*=\mathrm{NL2}^{+/+}, *=\mathrm{NL2}^{-/}$

Amplitudes (in $\mu \mathrm{V}$ ) of b-waves (A) and amplitude (in $\mu \mathrm{V}$ ) and frequency (in $\mathrm{Hz}$ ) of oscillatory potentials (B, C) of $\mathrm{NL2}^{-/-}$mice $(\mathrm{n}=8)$ in comparison with their $\mathrm{NL}^{+/+}$-littermates $(\mathrm{n}=9)$. a-waves were not detectable. Plotting against increasing light intensity in logarithmic scaling. Measurements shown here for $5 \mathrm{~ms}$ white flash light stimulus with 2 sec ISI. No significant differences in either latencies or frequencies were obtained. 
Photopic ERGs were only performed during the first set of experiments. The photopic responses were not as easy to deduce as the scotopic responses. In some cases, heavy baseline drifts made it difficult to isolate a true retinal signal from the baseline itself. An a-wave was not detectable. At higher light intensities though, especially with the longest stimulus we used (5ms), clear bwave responses appeared (Fig.21). With increasing light intensity, we were able to show that the photopic responses grow steadily in amplitude and that, from the detectable threshold on, the $\mathrm{NL}^{-{ }^{--}}$animals presented a trend towards reduced amplitudes which, however, was not statistically significant. b-wave latencies (data not shown) were not altered in the $\mathrm{NL}^{-/-}$. At highest stimuli, OPs were detectable, and showed a steady rise in both $\mathrm{NL}^{-/-}$and $\mathrm{NL}^{+/+}$. NL2 ${ }^{-/-}$OP amplitudes were smaller, but not statistically significant. OP frequencies were not altered in the NL2 ${ }^{-/}$. 


\subsubsection{Auditory function}

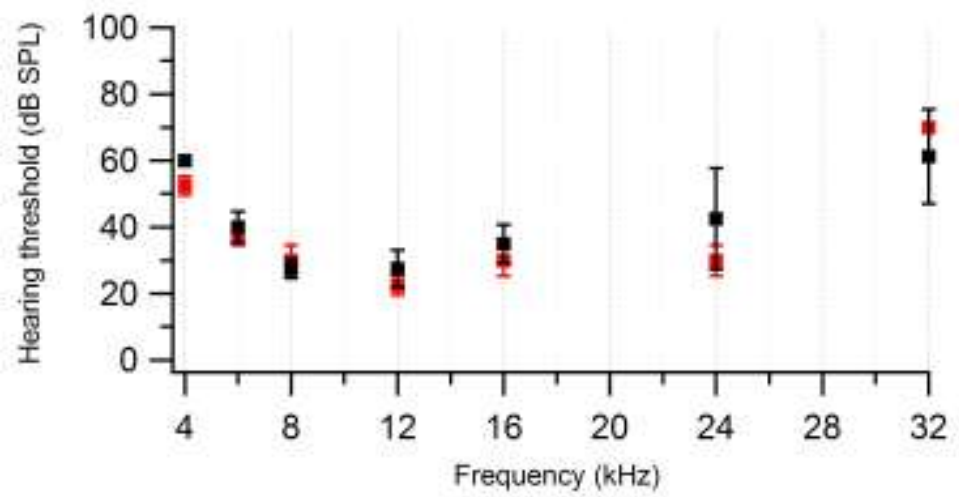

Figure 22: Frequency-specific hearing thresholds $\left(\mathrm{NL}^{-/}\right.$and $\left.\mathrm{NL}^{+/+}\right) . \cdot=\mathrm{NL2}^{+/+}, "=\mathrm{NL2}^{-/-}$
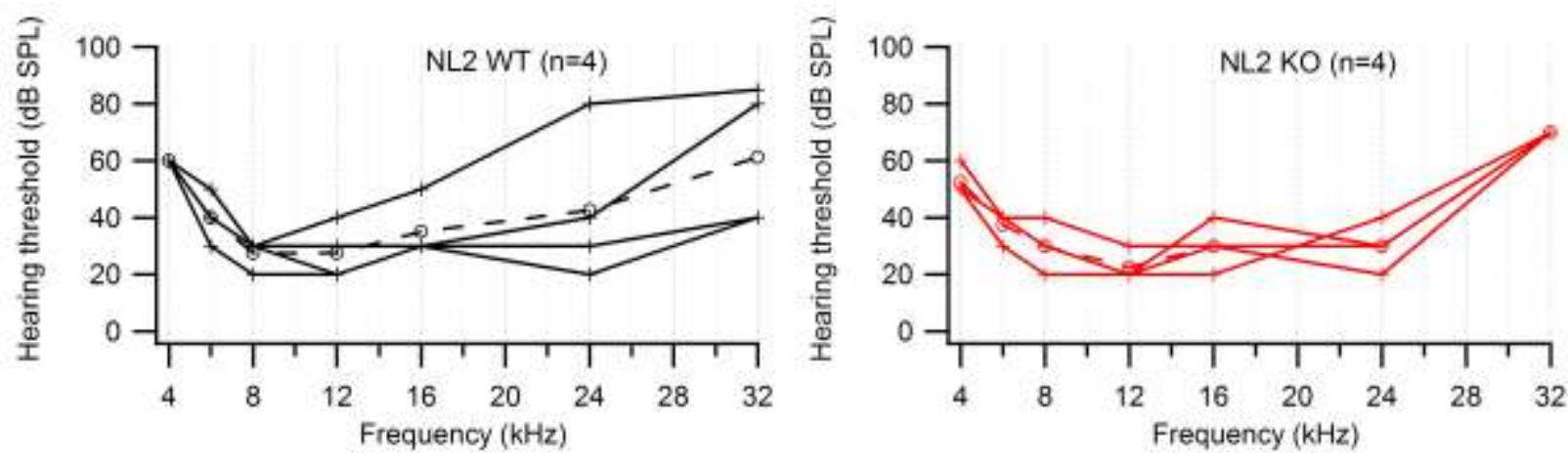

Figure 23: Ascertained individual and averaged audiograms $\left(\mathrm{NL}^{-/} \text {and } \mathrm{NL2}^{+/+}\right)_{+}+=\mathrm{NL2}^{+/+},+=\mathrm{NL}^{-/-}$

Fig.22 shows the mean values (with standard errors of means, SEM) of the frequency-specific hearing thresholds (in $\mathrm{dB}$ ) of $\mathrm{NL}^{+/+}$(black, $\left.\mathrm{n}=4\right)$ and $\mathrm{NL}^{-{ }^{--}}(\mathrm{red}, \mathrm{n}=4)$ mice in comparison. Detection was performed by measuring frequency-specific ABRs. Tested frequencies were 4, 6, 8, 12, 16, 24 and $32 \mathrm{kHz}$. No statistically significant difference in hearing thresholds was observed.

Figure 23 shows the ascertained audiograms of the same $N L 2^{+/+}$(left figure, black lines, $\left.n=4\right)$ and $\mathrm{NL}^{-/-}$mice (right figure, red lines, $\mathrm{n}=4$ ). Continuous lines show the individual animals' audiograms. Dashed lines show averaged audiogram curves of the respective animals. One wild type animal broke the range by showing very high hearing thresholds in higher frequencies. The knockout mice presented with very even hearing thresholds. In the mean, no significant differences in hearing thresholds could be observed between $\mathrm{NL}^{+/+}$and $\mathrm{NL} 2^{-/-}$. 


\section{A: $20 \mathrm{~Hz}$ click ABR wave I}
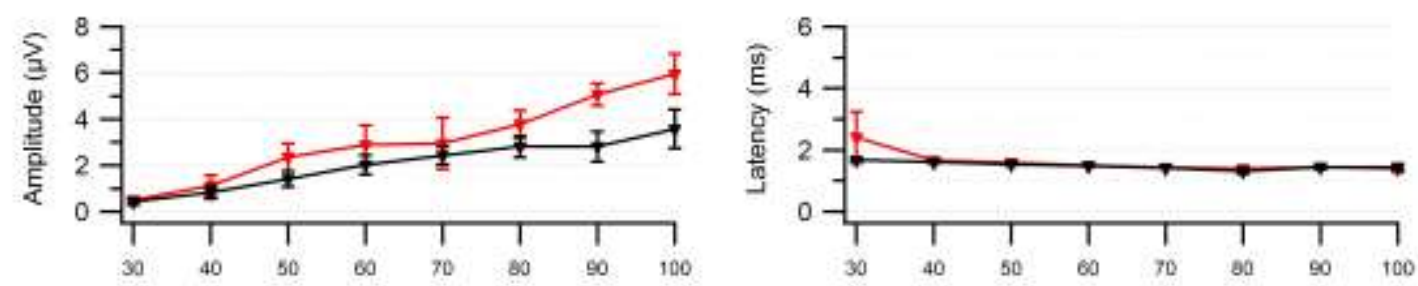

B: $20 \mathrm{~Hz}$ click ABR wave II
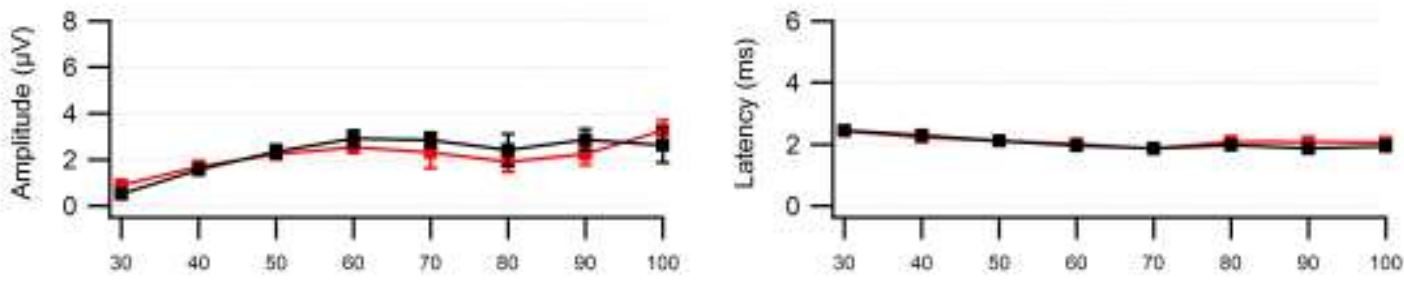

C: $20 \mathrm{~Hz}$ click ABR wave III
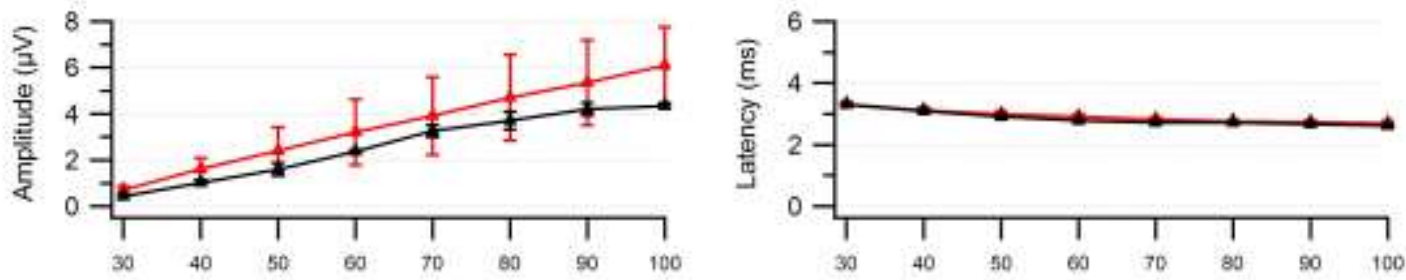

D: $20 \mathrm{~Hz}$ click ABR wave IV
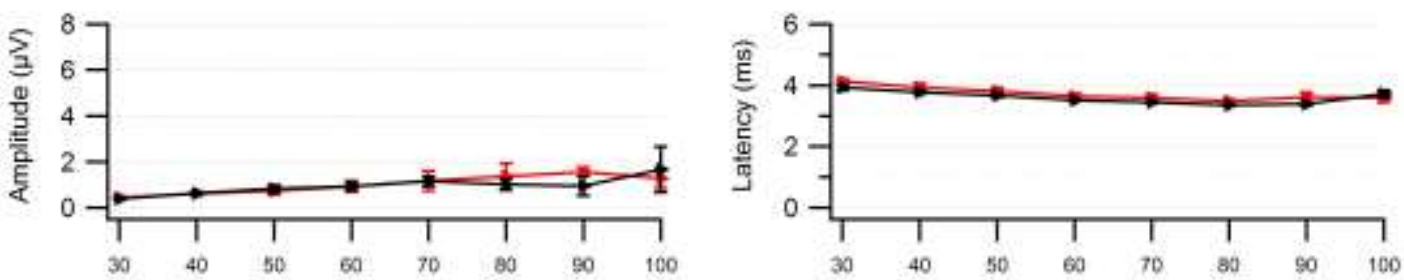

E: $20 \mathrm{~Hz}$ click ABR wave $\mathrm{V}$
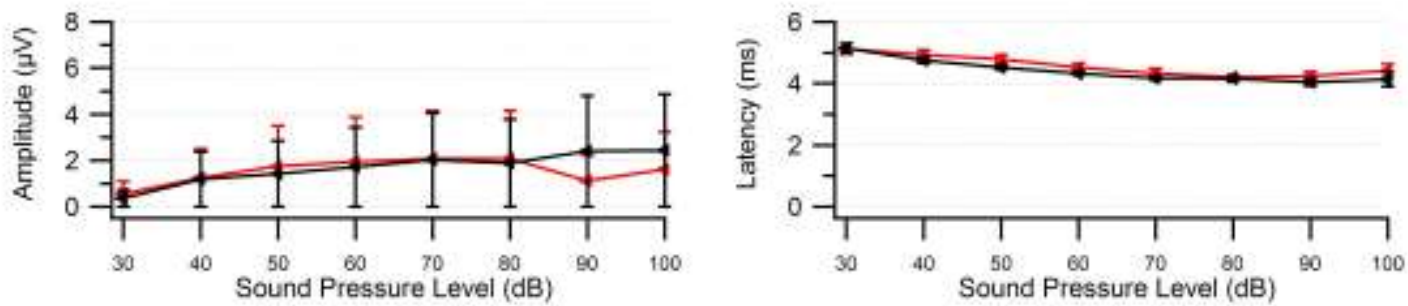

Figure 24: Single wave analysis of $20 \mathrm{~Hz}$ click ABR waves $\mathrm{JI}$ to $\mathrm{JV}\left(\mathrm{NL} 2^{-/-}\right.$and $\left.\mathrm{NL} 2^{+/+}\right) \bullet=\mathrm{NL} 2^{+/+}, \bullet=\mathrm{NL} 2^{-/-}$

ABRs measured with a $20 \mathrm{~Hz}$ click stimulus. $\mathrm{NL}^{+/+}$(black lines, $\mathrm{n}=4$ ) and $\mathrm{NL} 2^{-/-}$(red lines, $\mathrm{n}=4$ ). Left figures show the amplitudes of the waves (in $\mu \mathrm{V}$ ) at different sound pressure levels (in $\mathrm{dB}$ ). Right figures show the corresponding waves' latencies (in ms) for the same sound pressure levels (in $\mathrm{dB}$ ). No statistically significant differences in amplitudes or latencies of the ABR waves JI to JV were observed. 
Fig.24 shows the single waves of the ABR of four $\mathrm{NL}^{-/-}$and four $\mathrm{NL}^{+/+}$animals in comparison. The stimulus was always a $20 \mathrm{~Hz}$ click sound that was presented at multiple sound pressure levels. Amplitudes and latencies of the ABR waves JI to JV at every sound pressure level (30dB to $100 \mathrm{~dB}$ in steps of $10 \mathrm{~dB}$ ) are shown. The left panels show the amplitudes (in $\mu \mathrm{V}$ ) of the waves. The right panels show the corresponding latencies (in ms). No statistically significant difference in amplitudes nor latencies of the ABR waves was observed between $\mathrm{NL}_{2}^{-/-}$and $\mathrm{NL}_{2}^{+/+}$. 


\subsection{Protein 4.1 TKO}

Two sets of experiments were performed with the 4.1 TKO animals and their $4.1^{+/+}$littermates.

The first set of ERG measurements was performed using three different stimulus lengths $(0,1 \mathrm{~ms}$, $\mathrm{ms}$ and $5 \mathrm{~ms}$ ) and ISIs of 5 seconds for stimuli smaller than $1 \mathrm{cds} / \mathrm{m}^{2}$ and 17 seconds for stimuli from $1 \mathrm{cds} / \mathrm{m}^{2}$ on. This set of experiments showed good absolute amplitudes and partially statistically significant alteration between $4.1^{+/+-}$and 4.1 TKO animals $\left(n=9\right.$ for $4.1^{+/+-}$and $n=9$ for 4.1TKO). The second set of experiments was performed using the same ISIs but only the stimulus intensities according to the protocol suggested by Roland Consult electrophysiological diagnostic systems. In this protocol, only 10 light intensities at different stimulus lengths were tested. This set of experiments revealed larger absolute amplitudes and could reproduce the statistically significant differences between $4.1^{+/+-}$and 4.1 TKO animals $\left(\mathrm{n}=4\right.$ for $4.1^{+/+-}$and $\mathrm{n}=4$ for 4.1TKO). 


\subsubsection{Scotopic ERG measurements - Examples}

\subsubsection{First set of experiments}

A
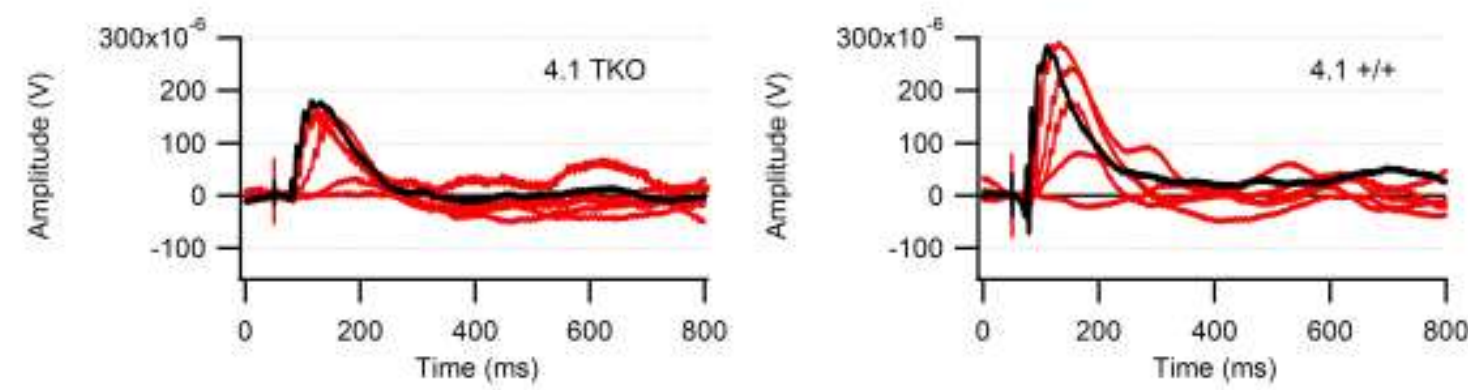

B
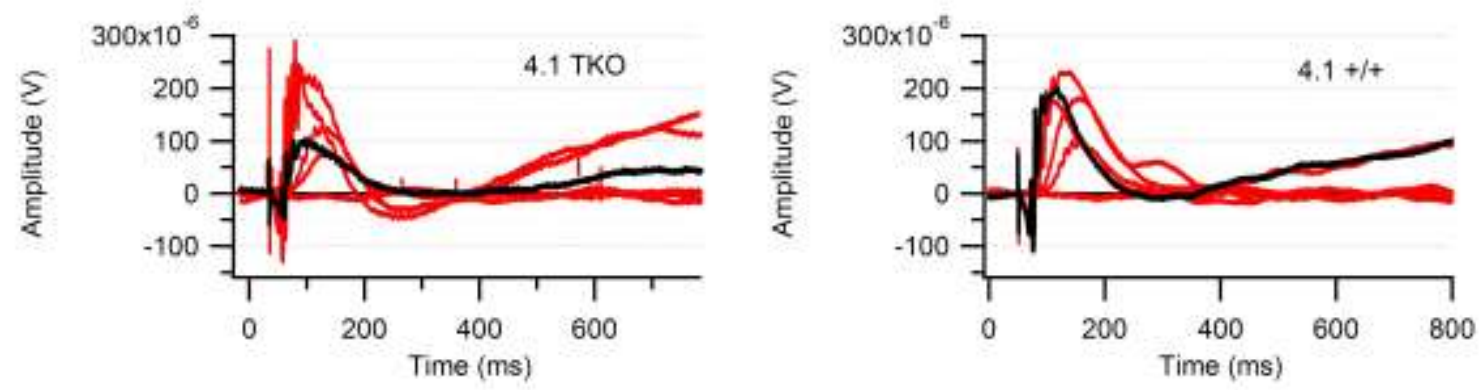

C
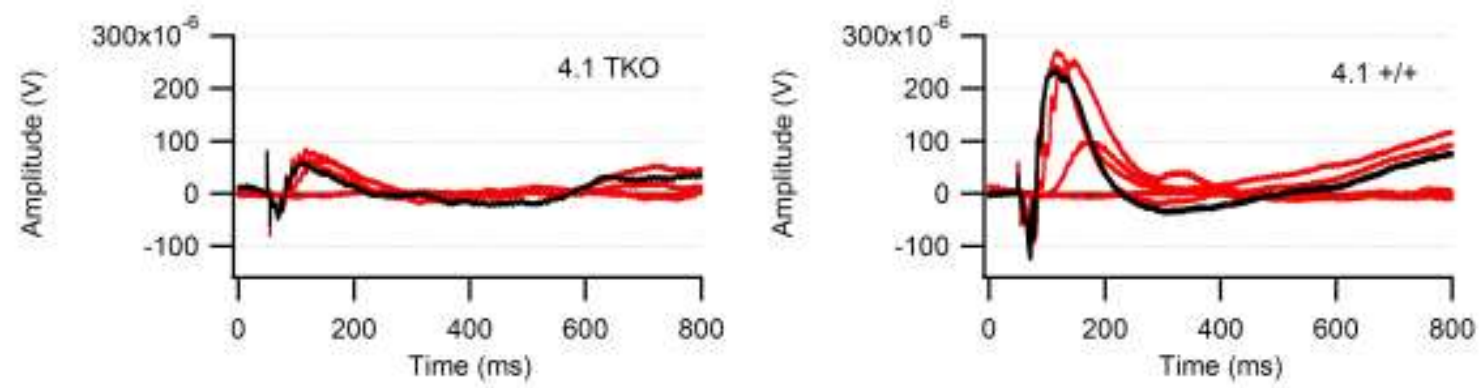

Figure 25: ERG examples of randomly chosen animals from the $1^{\text {st }}$ set of experiments $\left(4.1\right.$ TKO and $\left.4.1^{+/+}\right)$. $\bullet=4.1^{+/+}, \bullet=4.1 \mathrm{TKO}$

Examples of ERG waves of randomly chosen animals growing with increasing light intensity. 4.1TKO (left figures) and $4.1^{+/+}$(right figures). The 4.1TKO traces' amplitudes at all measured stimulus lengths are reduced in amplitude.

A: $0,1 \mathrm{~ms}$ stimulus, 5/17sec ISI, 10 averages per trace; B: $1 \mathrm{~ms}$ stimulus, $5 / 17 \mathrm{sec}$ ISI, 10 averages per trace; C: $5 \mathrm{~ms}$ stimulus, 5/17 sec ISI, 10 averages per trace; red lines show ERG traces at different attenuator steps, the black line is always the trace at highest light intensity (attenuator step 0 ). The flat red lines closest to the zero lines not showing observable ERG waves show subthreshold recordings in strongest flash attenuation.

Fig.25 shows examples of ERG recordings of randomly chosen animals (4.1TKO, left, and $4.1^{+/+}$, right). Fig.25A shows the experiments with a $0,1 \mathrm{~ms}$ stimulus, fig. $25 \mathrm{~B}$ with a $1 \mathrm{~ms}$ stimulus 
and fig. $25 \mathrm{C}$ with a $5 \mathrm{~ms}$ stimulus. The black line shows the ERG at maximum light intensity (Attenuation 0), the other lines demonstrate growth of amplitudes and shortening of latencies with increasing light intensity at different attenuation levels. A steady growth of the curves and shortening of latencies was observed in both, WT and TKO animals. The example showed clearly, how the TKO animals' ERG amplitudes (left figures) at maximum light intensities were much lower than the amplitudes of the wild types' (right figures). All experiments with the $4.1 \mathrm{TKO}$ animals and their littermates were performed with ISIs of $5 \mathrm{sec}$ for stimuli below $1 \mathrm{cds} / \mathrm{m}^{2}$ and 17 $\mathrm{sec}$ for stimuli from $1 \mathrm{cds} / \mathrm{m}^{2} \mathrm{on}$.

\subsubsection{Second set of experiments}
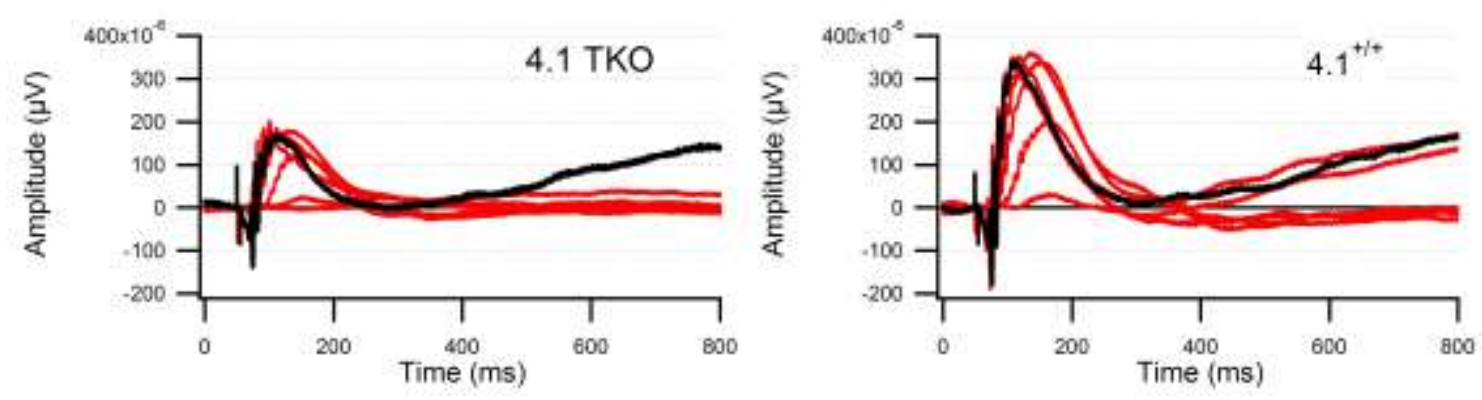

Figure 26: ERG examples of randomly chosen animals from the $2^{\text {nd }}$ set of experiments $\left(4.1\right.$ TKO and $\left.4.1^{+/+}\right)$. $\bullet=4.1^{+/+}, \bullet=4.1 \mathrm{TKO}$

Examples of ERG waves of randomly chosen animals growing with increasing light intensity. 4.1TKO (left figures) and $4.1^{+/+}$(right figures). The 4.1TKO traces' amplitudes at all measured stimulus lengths are reduced in amplitude.

$0,1 \mathrm{~ms}, 1 \mathrm{~ms}$ and $5 \mathrm{~ms}$ stimuli, $5 / 17 \mathrm{sec}$ ISI, Roland protocol, 10 averages per trace; red lines show ERG traces at different attenuator steps, the black line is always the trace at highest light intensity (attenuator step 0). The flat red lines closest to the zero lines not showing observable ERG waves show subthreshold recordings in strongest flash attenuation.

Fig.26 shows examples taken from the second set of experiments. A randomly chosen Protein 4.1 TKO (left) and Protein $4.1^{+/+}$(right) mouse measured according to the Roland Consult paradigm is presented. This paradigm does not consider different stimulus lengths, but only considers the resulting light intensity, which is why only one panel is shown here. Triple knockout and wildtype animal presented with significantly altered amplitudes in all acquired ERG traces. Absolute amplitudes were representative. 


\subsubsection{Amplitudes}

At all stimulus lengths $(0,1 \mathrm{~ms}, 1 \mathrm{~ms}$ and $5 \mathrm{~ms})$, mean values of amplitudes and latencies of awave and b-wave and mean values of amplitudes and frequencies of the oscillatory potentials were analyzed and plotted against their corresponding light intensities or time. The ERG recordings of Protein 4.1 TKO mice revealed in comparison with the corresponding Protein $4.1^{+/+}$control animals alterations in their retinal responses. 


\subsubsection{First set of experiments}

A

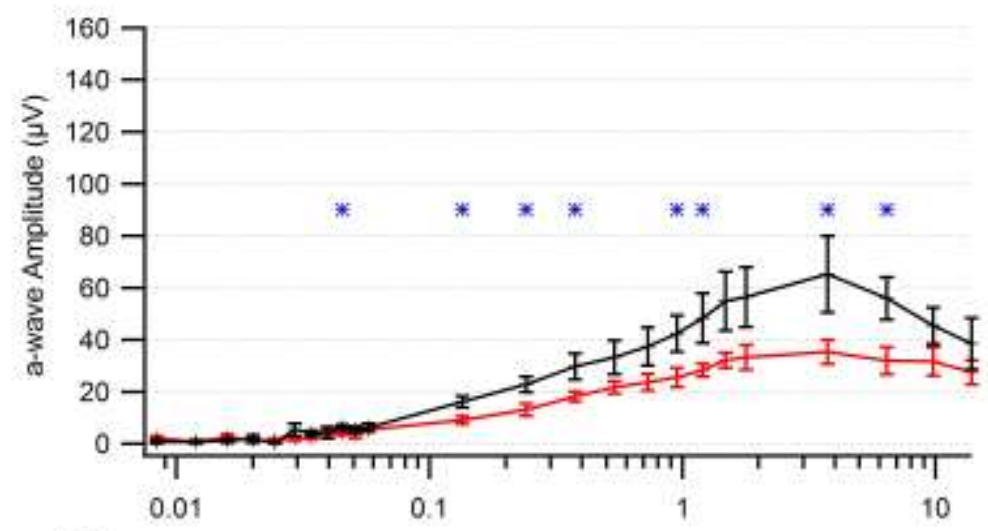

B

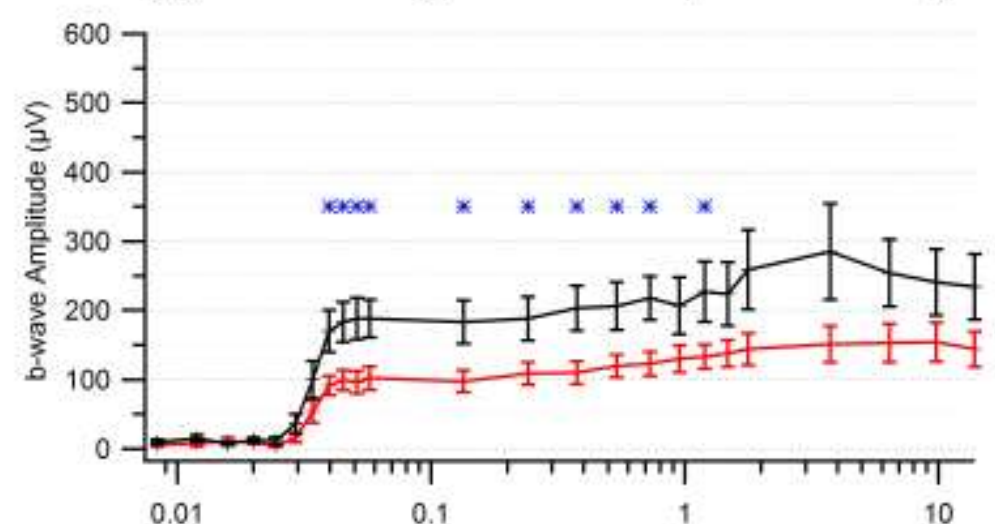

C

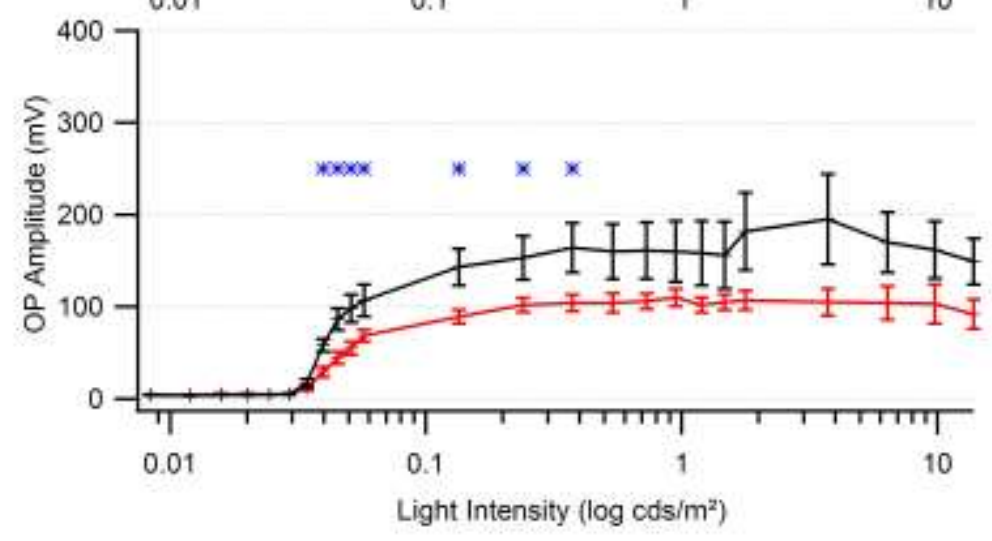

Figure 27: Amplitudes of a-waves, b-waves and OPs from the $1^{\text {st }}$ set of experiments $\left(4.1\right.$ TKO and $\left.4.1^{+/+}\right)$

$*=\mathrm{p} \leq 0,05, *=4.1^{+/+}, *=4.1 \mathrm{TKO}$

Amplitudes (in $\mu \mathrm{V}$ ) of a-waves (A), b-waves (B) and oscillatory potentials (C) of 4.1TKO mice (n=9) in comparison with their WT-littermates $(n=9)$. Measurements shown here for $5 \mathrm{~ms}$ white flash light stimulus with 5 sec ISI for light intensities below $1 \mathrm{cds} / \mathrm{m}^{2}$ and $17 \mathrm{sec}$ for light intensities from $1 \mathrm{cds} / \mathrm{m}^{2} . *=\mathrm{p} \leq 0,05$. Plotting against increasing light intensity in logarithmic scaling. 
Clear a-waves could be evoked with all stimulus lengths $(0,1 \mathrm{~ms}$ and $1 \mathrm{~ms}$ stimuli not shown here). The a-waves showed a steady rise in amplitude with increasing light intensity. A consistent suprathreshold reduction of the a-wave amplitudes was observed in the 4.1TKO animals, which was statistically significant at most light intensities for the $5 \mathrm{~ms}$ stimulus (Fig.27A). The 4.1TKO animals' amplitudes reached a plateau, but did not show a clear decline at maximum stimuli. In contrast, the WT animals rose steadily and then declined without a plateau in between.

The b-wave amplitudes (Fig.27B) showed a steady rise at low light intensities. Good responses were recorded at all stimulus lengths. The 4.1TKO animals presented with reduced amplitudes above threshold at all stimulus lengths. Significant reduction was observed at medium light intensities for the $5 \mathrm{~ms}$ stimulus. A plateau in the b-wave amplitudes demarked soon above threshold in both $4.1^{+/+}$and $4.1 \mathrm{TKO}$

For the oscillatory potentials, a general reduction of the amplitudes was seen in the 4.1TKO animals for all stimulus lengths at light intensities above threshold (Fig.27C). From threshold on, the amplitudes rose steadily, reaching a plateau at medium light intensities. An obvious decline in both $4.1^{+/+}$and 4.1 TKO was not detectable. Statistically significant alterations were seen at lower to medium light intensities for the $5 \mathrm{~ms}$ stimulus. 


\subsubsection{Second set of experiments}

A

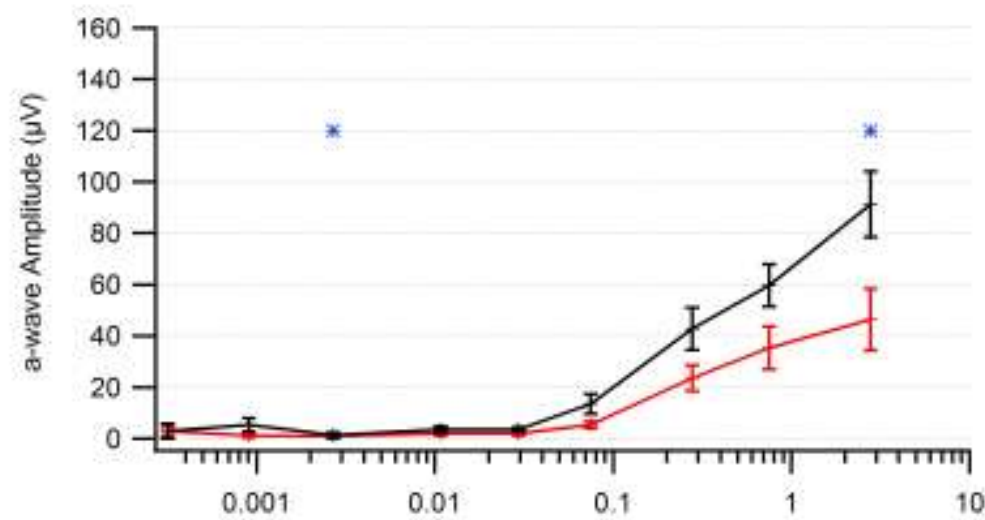

B
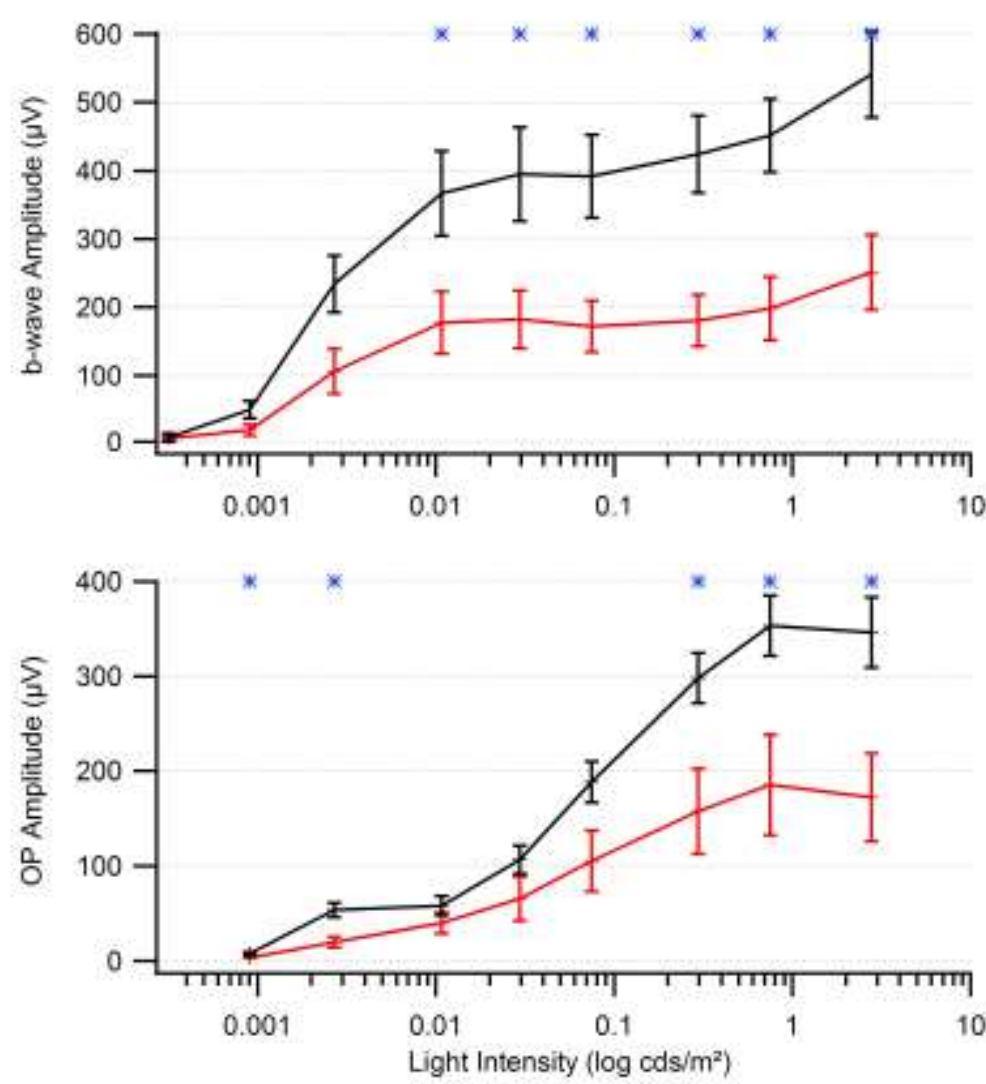

Figure 28: Amplitudes of a-waves, b-waves and OPs from the $2^{\text {nd }}$ set of experiments $\left(4.1\right.$ TKO and $\left.4.1^{+/+}\right)$

$*=\mathrm{p} \leq 0,05, *=4.1^{+/+}, *=4.1 \mathrm{TKO}$

Amplitudes (in $\mu \mathrm{V}$ ) of a-waves (A), b-waves (B) and oscillatory potentials $(\mathrm{C})$ of $4.1 \mathrm{TKO}$ mice $(\mathrm{n}=4)$ in comparison with their $4.1^{+/+}$-littermates $(n=4)$. Measurements shown here for different stimulus lengths with a 5 sec ISI for light intensities below $1 \mathrm{cds} / \mathrm{m}^{2}$ and $17 \mathrm{sec}$ ISI for light intensities from $1 \mathrm{cds} / \mathrm{m}^{2}$. $*=\mathrm{p} \leq 0,05$. Plotting against increasing light intensity in logarithmic scaling $\left(\log \mathrm{cds} / \mathrm{m}^{2}\right)$. 
In the second set of ERG measurements performed with the protocol adapted from the Q450 Ganzfeld program, clear a-waves, b-waves and oscillatory potentials were recorded (Fig.28). In all ERG components, an obvious reduction of amplitudes was observed. From threshold on, the 4.1TKO animals presented with lower a-wave amplitudes (Fig.28A). The a-wave amplitudes were reduced statistically significant at the highest light intensity.

The b-waves could be recorded already at fairly low light intensities (Fig.28B). From threshold on, the Protein 4.1 TKO animals presented with statistically significant reduction in b-wave amplitudes at all light intensities.

The oscillatory potentials appeared at the same time as the b-waves (Fig.28C). Also, from the lowest light intensity on, they were lower than the OPs of the wild type animals. The difference reached statistical significance at the highest presented light intensities. While the b-wave amplitudes did not reach a plateau at medium or higher light intensities, the OPs showed a certain saturation at maximum light intensity. 


\subsubsection{Temporal components}

\subsubsection{First set of experiments}

A

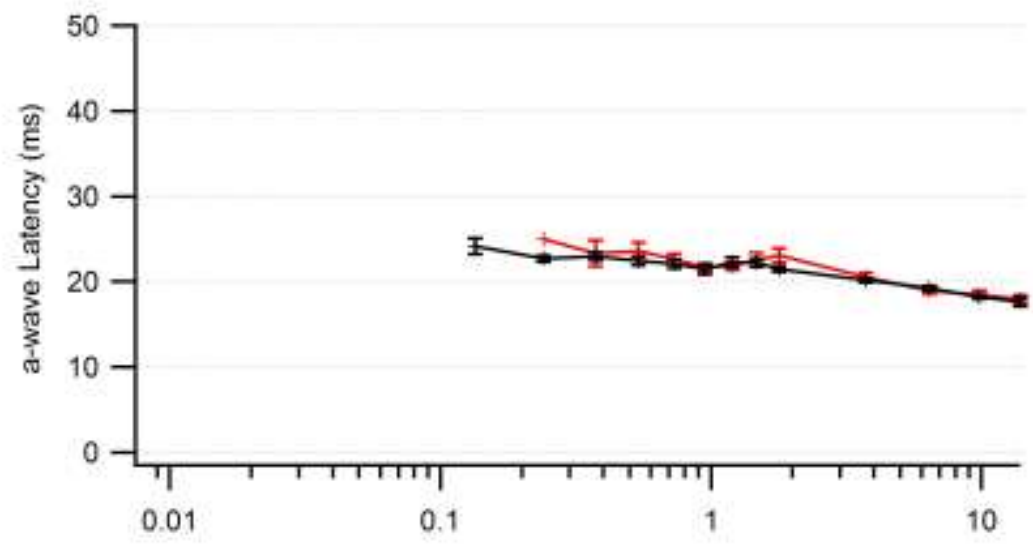

B

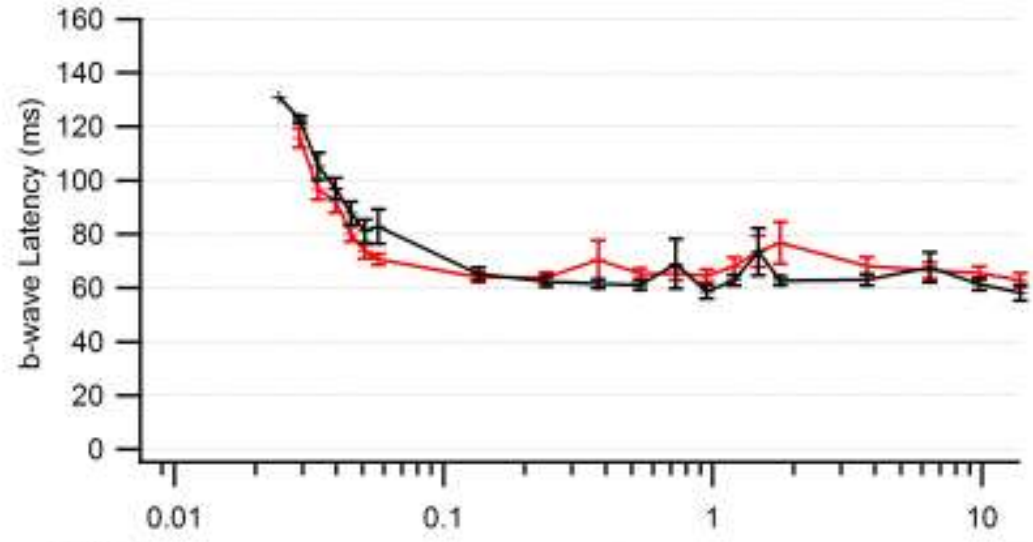

C

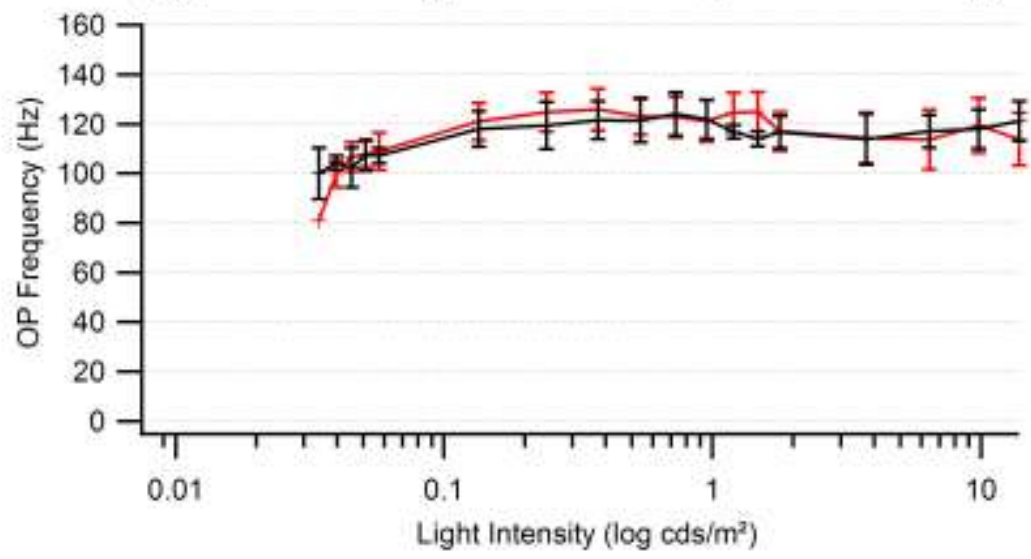

Figure 29: Latencies of a-waves, b-waves and frequencies of OPs from $1^{\text {st }}$ set of experiments (4.1 TKO and $\left.4.1^{+/+}\right)$

$*=4.1^{+/+}, *=4.1 \mathrm{TKO}$

Latencies (in ms) of a-waves (A), b-waves (B) and frequency of oscillatory potentials (C) of 4.1TKO mice ( $\mathrm{n}=9$ ) in comparison with their WT-littermates $(n=9)$. Measurements shown here for $5 \mathrm{~ms}$ white flash light stimulus with 5 sec ISI for light intensities below $1 \mathrm{cds} / \mathrm{m}^{2}$ and $17 \mathrm{sec}$ for light intensities above $1 \mathrm{cds} / \mathrm{m}^{2} . *=\mathrm{p} \leq 0,05$ Plotting against increasing light intensity in logarithmic scaling. 


\subsubsection{Second set of experiments}

A

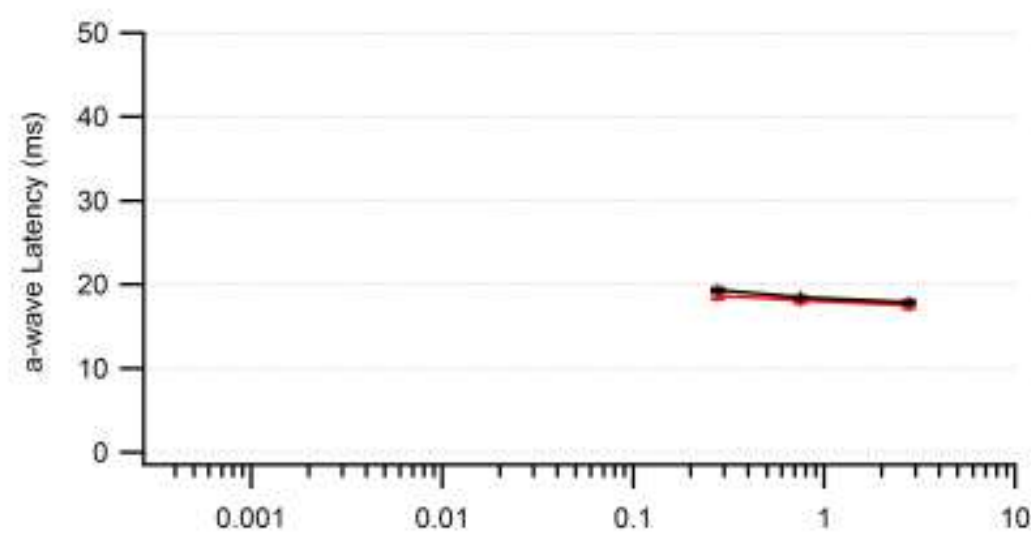

B

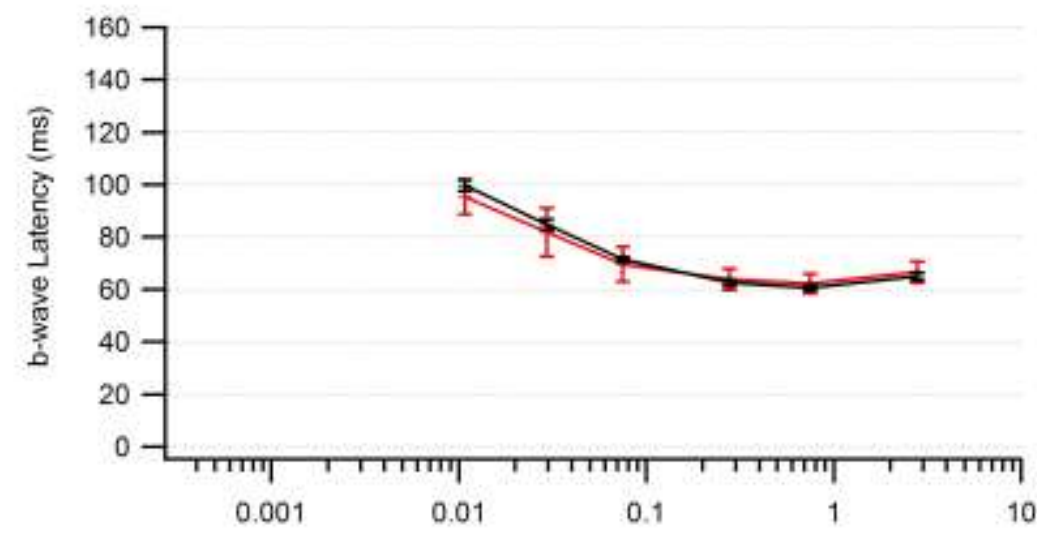

C

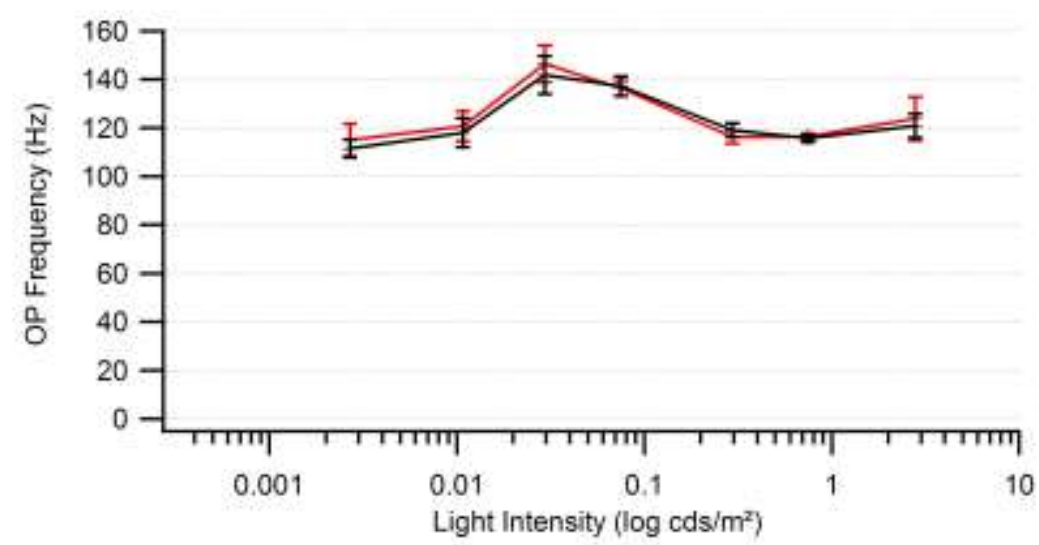

Figure 30: Latencies of a-waves, b-waves and frequencies of OPs from $2^{\text {nd }}$ set of experiments (4.1 TKO and $\left.4.1^{+++}\right)$

$*=4.1^{+/+}, *=4.1 \mathrm{TKO}$

Latencies (in ms) of a-waves (A), b-waves (B) and frequency of oscillatory potentials (C) of 4.1TKO mice ( $\mathrm{n}=4)$ in comparison with their WT-littermates $(\mathrm{n}=4)$. Measurements shown here for different stimulus lengths with a 5 sec ISI for stimuli below $1 \mathrm{cds} / \mathrm{m}^{2}$ and $17 \mathrm{sec}$ ISI for stimuli above $1 \mathrm{cds} / \mathrm{m}^{2} . *=\mathrm{p} \leq 0,05$ Plotting against increasing light intensity in logarithmic scaling. 
In both sets of experiments (Fig.29 and 30), a- and b-wave latencies were not altered in the 4.1TKO. The latencies shortened with increasing light intensity. Neither did the 4.1TKO animals present with alterations in their OP frequencies compared to their WT littermates. In contrast, they appeared to be very stable in both animal groups over the whole range of light intensities at all stimulus lengths. 


\subsubsection{Photopic ERG measurements}

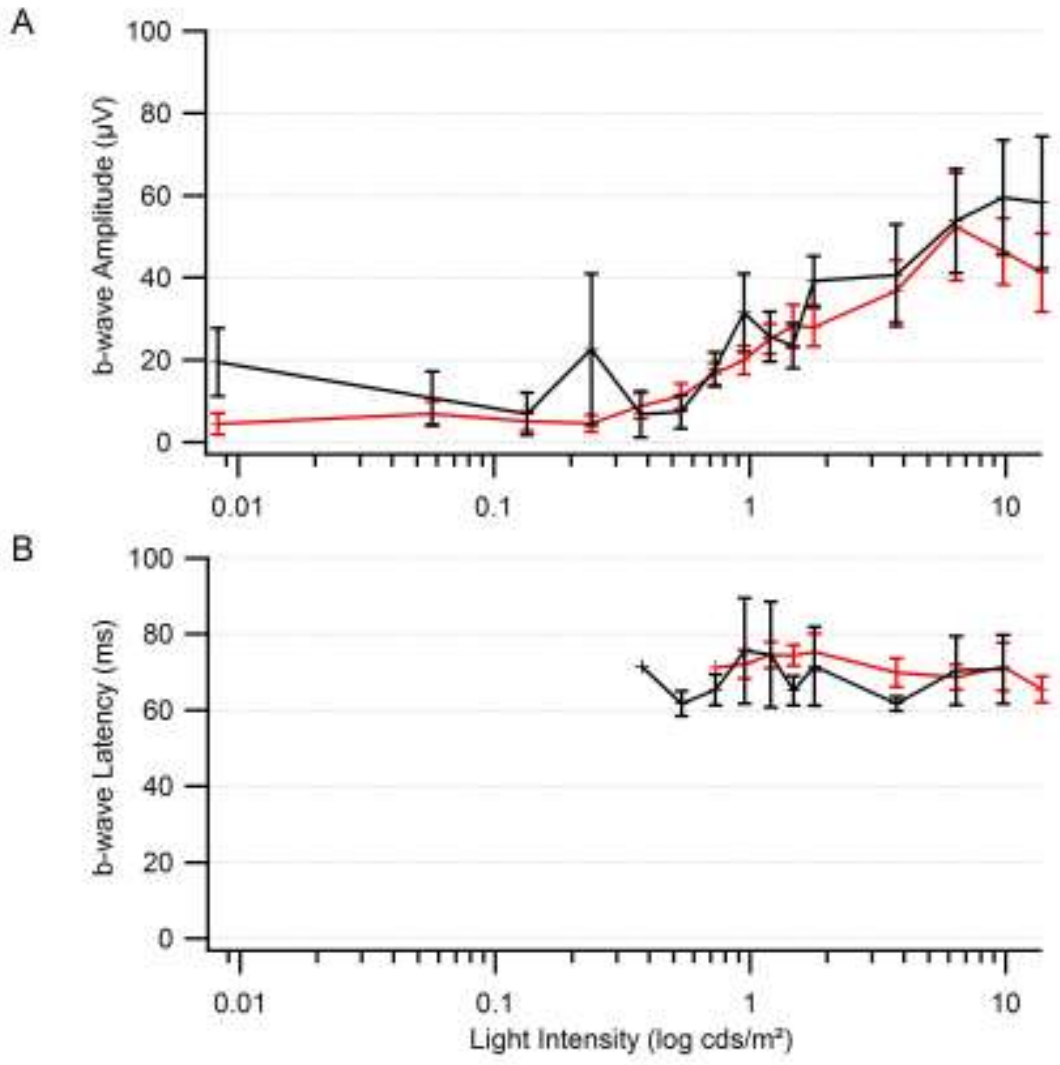

Figure 31: Photopic b-wave amplitudes and latencies fom the $1^{\text {st }}$ set of experiments $\left(4.1 \mathrm{TKO}\right.$ and $\left.4.1^{+/+}\right)$

$*=4.1^{+/+}, *=4.1$ TKO

Amplitudes (in $\mu \mathrm{V}$ ) (A) and latencies (B) (in ms) of b-waves of 4.1TKO mice ( $\mathrm{n}=9$ ) in comparison with their WTlittermates $(n=9)$. a-waves and oscillatory potentials were not clearly detectable. Plotting against increasing light intensity in logarithmic scaling). Measurements shown here for $5 \mathrm{~ms}$ white flash light stimulus with 5 sec ISI for light intensties below $1 \mathrm{cds} / \mathrm{m}^{2}$ and $17 \mathrm{sec}$ for light intensities above $1 \mathrm{cds} / \mathrm{m}^{2} . *=\mathrm{p} \leq 0,05$ Plotting against increasing light intensity in logarithmic scaling $\left(\log \mathrm{cds} / \mathrm{m}^{2}\right)$.

Photopic ERGs were only performed during the first set of experiments. The photopic responses were not as easy to deduce as the scotopic responses. An a-wave was not detectable. At high light intensities, b-wave responses appeared. No statistically significant difference in the photopic responses' amplitudes (Fig.31A) or latencies (Fig.31B) was observed between the 4.1TKO and 4.1WT animals. OPs were not detectable. 


\subsubsection{Auditory function}

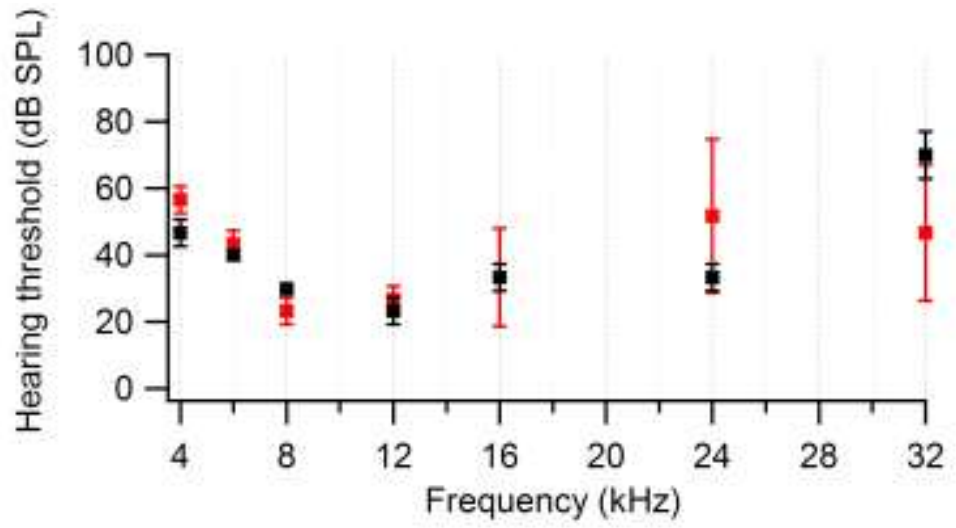

Figure 32: Frequency-specific hearing thresholds $\left(4.1 \mathrm{TKO}\right.$ and $\left.4.1^{+/+}\right) \cdot=4.1^{+/+}, "=4.1$ TKO
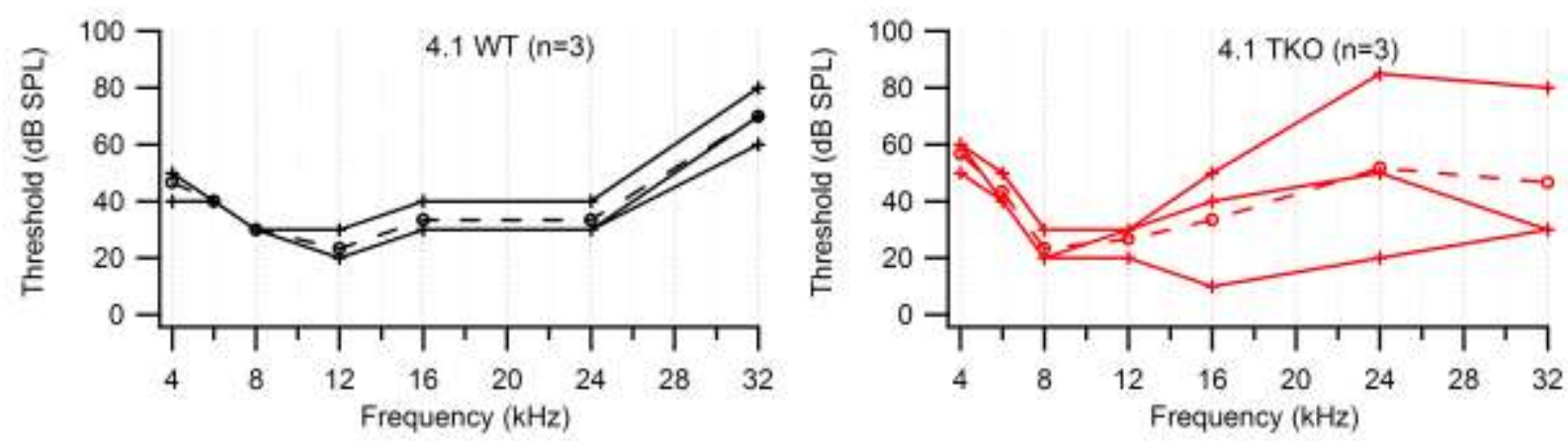

Figure 33: Ascertained individual and averaged audiograms $\left(4.1 \mathrm{TKO}\right.$ and $\left.4.1^{+/+}\right)+=4.1^{+/+},+=4.1 \mathrm{TKO}$

Fig.32 shows the frequency-specific hearing thresholds (in $\mathrm{dB}$ ) with standard errors of the means of Protein $4.1^{+/+}$(black, $n=3$ ) and Protein 4.1 TKO (red, $n=3$ ) mice in comparison. Tested frequencies were 4, 6, 8, 12, 16, 24 and $32 \mathrm{kHz}$. No statistically significant difference was observed. Standard error of the means in the $4.1 \mathrm{TKO}$ animals was high.

Figure 33 shows the ascertained individual and averaged audiograms of the same 4.1 WT animals (left figure, black lines, $n=3$ ) and their 4.1 TKO littermates (right figure, red lines, $n=3$ ). Continuous lines show the individual animals' audiograms. Dashed lines show averaged audiogram curves of the respective animals. The wildtype mice presented with very even hearing and reproducible hearing thresholds. The knockout animals showed more individually differing audiograms. No continuity especially in higher frequencies was observed. In the mean, no significant differences in hearing thresholds could be observed between Protein $4.1^{+/+}$and Protein 4.1 TKO mice. 
A: $20 \mathrm{~Hz}$ click ABR wave I
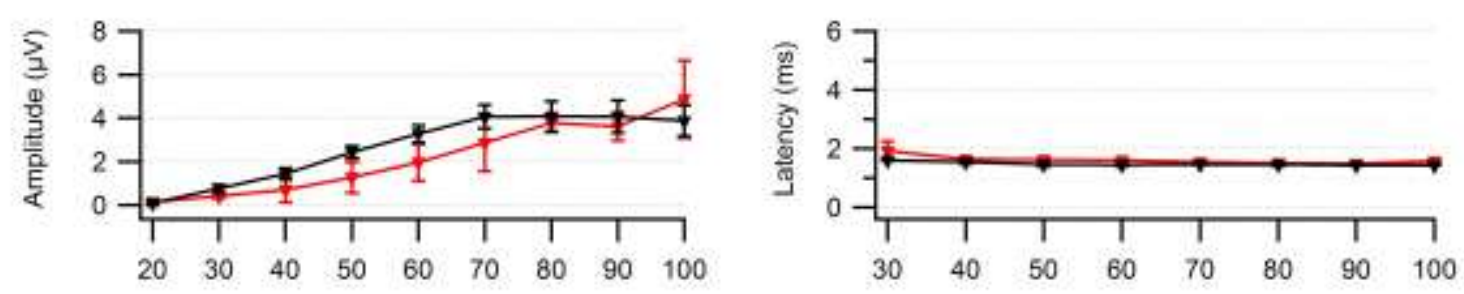

B: $20 \mathrm{~Hz}$ click ABR wave II
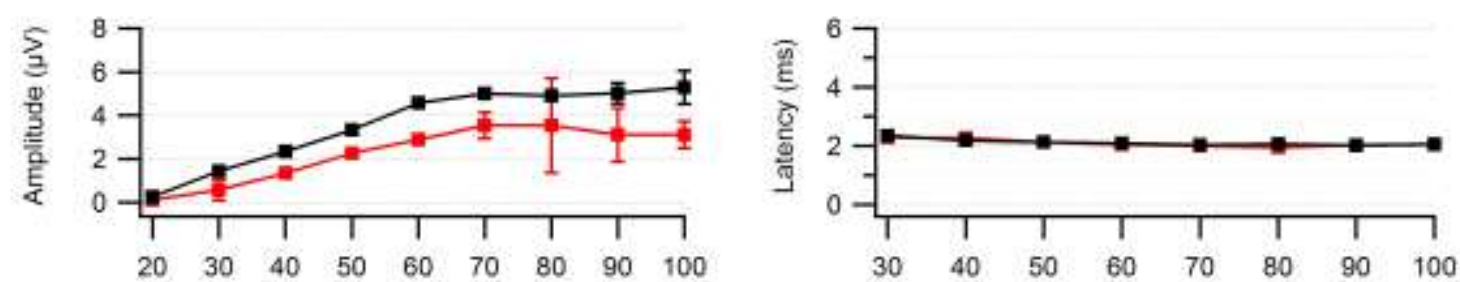

C: $20 \mathrm{~Hz}$ click $\mathrm{ABR}$ wave III
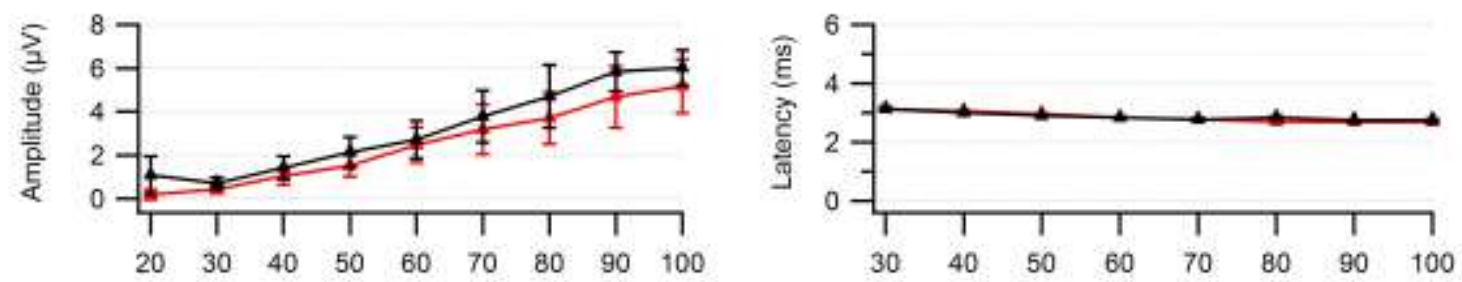

D: $20 \mathrm{~Hz}$ click ABR wave IV
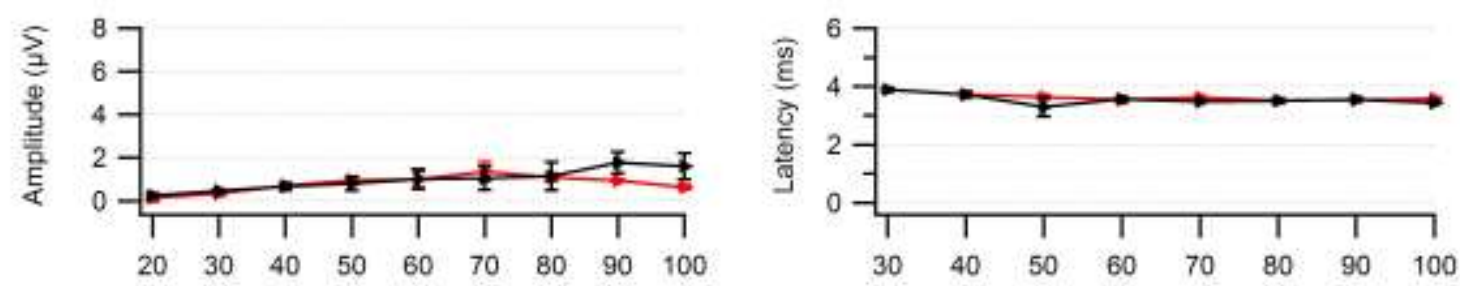

E: $20 \mathrm{~Hz}$ click ABR wave $\mathrm{V}$
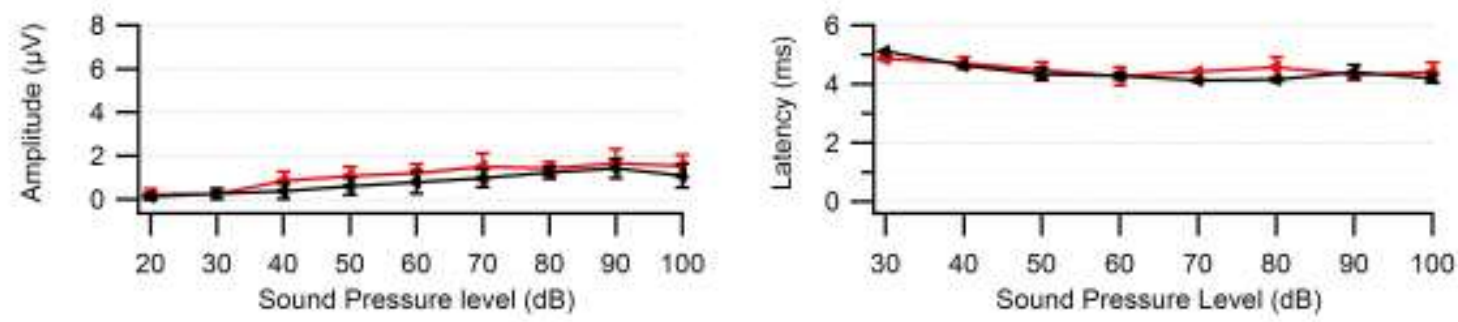

Figure 34: Single wave analysis of ABR waves JI to JV $\left(4.1 \mathrm{TKO}\right.$ and $\left.4.1^{+/+}\right) \bullet=4^{+/+}, \bullet=4.1 \mathrm{TKO}$

Threshold waves for ABR waves JI (A) to JV (E) measured with a $20 \mathrm{~Hz}$ click stimulus. $4.1^{+/+}$(black lines, $\mathrm{n}=3$ ) and 4.1TKO (red lines, $\mathrm{n}=3$ ).Left panels show the amplitudes of the threshold waves $($ in $\mu \mathrm{V}$ ) at different sound pressure levels (in $\mathrm{dB}$ ). Right panels show the corresponding waves' latencies (in ms) for the same sound pressure levels (in dB). 
Fig.34 shows the single waves of the ABR of three 4.1 TKO mice nd three Protein 4.1 WT mice in comparison. The stimulus was always a $20 \mathrm{~Hz}$ click sound. Amplitudes and latencies of the ABR waves JI to JV at every sound pressure level (30dB to $100 \mathrm{~dB}$ in steps of $10 \mathrm{~dB}$ ) are shown. The left panels show the amplitudes (in $\mu \mathrm{V}$ ) of the waves. The right panels show the corresponding latencies (in ms). No statistically significant difference was observed between Protein 4.1 TKO and Protein 4.1 WT animals. 


\section{Discussion}

\subsection{ERG}

Interpretation of the ERG requires the identification of the contributions of the many different cell types to its components. This way, the ERG can be analysed and observed alterations can be attributed to retinal elements. The ERG is a sum potential of several temporally overlapping components. Studies have been carried out to dissect the individual components of the ERG and their specific course in time e.g. by Robson and Frishman, 1998-1999.

\subsubsection{Considerations on our ERG experiments}

In this work, we established a customized protocol for ERG recordings in anaesthetized live mice. Performing these experiments on the different mutant mice, we first of all wanted to explore the overall retinal function of the NL2 and Protein 4.1 mutant mice. Do these animals see? Is there a gross impairment of either scotopic or photopic vision? How do the animals react to different stimulus lengths or inter-stimulus-intervals? Are there obvious alterations in the main components of the ERG? The functional experiments were interpreted in relation to concomitantly performed morphological analyses of the mutant retinae.

\subsubsection{Variability of mouse ERG recordings}

Comparing our protocol and three other protocols of other working groups (table 5), it is obvious that the single parameters and tools used for the ERG recordings differ a lot within the groups. 


\begin{tabular}{|c|c|c|c|c|c|}
\hline$\underline{\text { Author }}$ & $\frac{\text { Dark Adapta- }}{\underline{\text { tion Time }}}$ & $\begin{array}{l}\frac{\text { Anaesthetic }}{\text { and }} \\
\text { Application }\end{array}$ & $\underline{\text { Mydriatic }}$ & $\begin{array}{c}\text { Reference and } \\
\text { grounding electrodes }\end{array}$ & $\underline{\text { Signal recording electrode }}$ \\
\hline$\frac{\text { Dick, O. et al. }}{\underline{(2003)}}$ & At least $12 \mathrm{hrs}$ & $\begin{array}{l}\text { Ketamine } \\
\text { Xylazine } \\
\text { Intraperitoneally }\end{array}$ & $\begin{array}{l}1 \% \text { atropine } \\
\text { sulfate }\end{array}$ & Needle electrodes & $\begin{array}{l}\mathrm{Ag} / \mathrm{AgCl} \text { corneal electrode } \\
\text { Moistened cotton wick }\end{array}$ \\
\hline $\begin{array}{l}\text { Saszik et al. } \\
\underline{(2002)}\end{array}$ & Overnight & $\begin{array}{l}\text { Ketamine } \\
\text { Xylazine } \\
\text { Intraperitoneally }\end{array}$ & $\begin{array}{l}0,5 \% \text { topical } \\
\text { atropine }\end{array}$ & $\begin{array}{l}\text { Grounding electrode: } \\
\text { wire lasso hooked be- } \\
\text { hind the upper front } \\
\text { teeth }\end{array}$ & $\begin{array}{l}\text { DTL fiber electrodes placed } \\
\text { on both eyes } \\
1.2 \% \text { saline-moistened } 1.2 \% \\
\text { methylcellulose } \\
\text { Stimulated eye covered with } \\
\text { ALCAR-film contact lens } \\
\text { Non-stimulated eye covered }\end{array}$ \\
\hline $\begin{array}{l}\text { Jaissle et al. } \\
\underline{(2001)}\end{array}$ & $\begin{array}{l}\text { Overnight } \\
\text { At least } 6 \text { hrs }\end{array}$ & $\begin{array}{l}\text { Ketamine } \\
\text { Xylazine } \\
\text { Atropine } \\
\text { Subcutaneously }\end{array}$ & $\begin{array}{l}\text { Tropicamide } \\
\text { Phenylephrine }\end{array}$ & $\begin{array}{l}\text { Silver needle elec- } \\
\text { trodes }\end{array}$ & $\begin{array}{l}\text { Gold wire ring electrodes } \\
\text { Moistened methylcellulose }\end{array}$ \\
\hline$\frac{\text { InnerEarLab }}{\underline{(2006-\text { now })}}$ & $\begin{array}{l}\text { Overnight } \\
\text { At least } 12 \mathrm{hrs}\end{array}$ & $\begin{array}{l}\text { Ketamine } \\
\text { Xylazine } \\
\text { Intraperitoneally }\end{array}$ & $\begin{array}{l}1 \% \text { atropine } \\
\text { sulfate }\end{array}$ & $\begin{array}{l}\text { Chromium-nickel- } \\
\text { steel canules }\end{array}$ & $\begin{array}{l}\mathrm{Ag} / \mathrm{AgCl} \text { corneal electrode } \\
\text { Moistened with electrode gel, } \\
\text { Agar-Ringer-block or } \\
\text { methylcellulose }\end{array}$ \\
\hline
\end{tabular}

\begin{tabular}{|c|c|c|c|c|c|}
\hline Author & $\underline{\text { Stimulus intensity }}$ & $\underline{\text { Stimulus }}$ & Inter-Stimulus-Interval & $\frac{\text { Number of steps from }}{\frac{\text { darkness to full light }}{\underline{\text { intensity }}}}$ & $\begin{array}{l}\text { Number of averag- } \\
\text { es of single traces }\end{array}$ \\
\hline$\underline{\text { Dick, O. et al. }}$ & & $20 \mathrm{~ms}$ & $10 \mathrm{sec}$ & & $16-32$ \\
\hline $\begin{array}{l}\text { Saszik et al. } \\
\underline{(2002)}\end{array}$ & $-6.1--0.5 \log \mathrm{sc} t \mathrm{td}$ & $\begin{array}{l}0.8 \mu \mathrm{s} \\
4.1 \mathrm{~ms}\end{array}$ & & & $\begin{array}{l}\text { Many times for } \\
\text { weak flashes } \\
\text { Fewer times for } \\
\text { stronger flashes }\end{array}$ \\
\hline $\begin{array}{l}\text { Jaissle et al. } \\
\underline{(2001)}\end{array}$ & $10^{-4}-25 \mathrm{cds} / \mathrm{m}^{2}$ & & $\begin{array}{l}5 \mathrm{sec}<1 \mathrm{cds} / \mathrm{m}^{2} \\
17 \mathrm{sec}>1 \mathrm{cds} / \mathrm{m}^{2}\end{array}$ & $\begin{array}{l}10 \text { steps } \\
(0.5 \text { and } 1 \log \mathrm{cds} / \mathrm{m})^{2}\end{array}$ & $5-10$ \\
\hline$\frac{\text { InnerEarLab }}{(2006-\text { now })}$ & $0.00017-13.9 \mathrm{cds} / \mathrm{m}^{2}$ & $\begin{array}{l}0.1 \mathrm{~ms} \\
1 \mathrm{~ms} \\
5 \mathrm{~ms}\end{array}$ & $\begin{array}{l}\text { Earlier: } 2 \mathrm{sec} \\
\text { Present: } 5 \mathrm{sec}<1 \mathrm{cds} / \mathrm{m}^{2} \\
17 \mathrm{sec} \geq 1 \mathrm{cds} / \mathrm{m}^{2}\end{array}$ & $\begin{array}{l}24 \text { (scotopic) } \\
15 \text { (photopic) } \\
\text { reduced to } 10 \text { (scotopic) }\end{array}$ & 10 \\
\hline
\end{tabular}

Table 4: Selection of different scotopic ERG protocols by different study groups 
Several critical factors contribute to the retinal response to light signals. Many factors such as stimulus intensity, light wavelength, background illumination, duration and spatial extent of the stimulus and location of the stimulus within the visual field can determine the relative magnitude of the ERG components (Robson and Frishman, 1998-1999).

Many different ERG protocols are presented within literature and the field has not yet agreed on a "gold standard" on how to perform electroretinography. The experimental protocol either needs to be specialized to stimulate the retinal structures of interest or stimulate the whole retina to deduce a sum potential for an overview retinal response.

One of the most sensitive factors is the corneal electrode. Interestingly, this recording electrode differs among the mentioned groups measuring ERGs (table 4). Two other sensitive factors are the stimulus duration and the inter-stimulus-intervals.

Robson and Frishman stated in 1998-1999 that the interpretation of the ERG in terms of its basic components is difficult if attention is limited to times and amplitudes of the obvious peaks and troughs in the records. From analyzing studies on the ERG components, they consider it generally more useful to take the amplitude of the ERG at various fixed times after a stimulus flash. Here, we analyse the sum potential and deduce our hypotheses on retinal function or malfunction from the obvious peaks and troughs in the wave. We interpret our results in relation with morphological findings of the mutant retina.

Pinto et al. constituted in 2007 three principal reasons why the ERG recorded from a mouse might differ among experiments: Firstly, the background strain of the mouse might differ from that of the "normal" mouse that the experimenter has in mind. Secondly, technical difficulties associated with inadvertent light-adaptation, electrode placement and stimulus and adapting levels may occur. Last but not least it is possible that a mouse with an abnormal ERG, confirmed by retesting to eliminate technical difficulties as the culprit, has a mutation that is responsible for the phenotype. Only by breeding for two generations and testing 20 or more second generation progeny can the latter possibility be examined further.

On performing our ERG measurements, we did collide with mainly the technical difficulties mentioned by Pinto et al. in 2007. As these are all factors mainly dependent on the person performing the experiments, the human factor is a variable. This variable can only be minimized, if not turned into a constant, if experimenters in a lab act in the same set, after determined protocols and standardized animal handling and preparatory procedures. The experimenters, whose re- 
sults are shown in this work, have all been instructed by the same person and performed most of the experiments together. The results that were not well reproducible (especially for the NL2 animals), were gained from experiments with different protocols. The technical systems were checked several times without ruling out a technical error. The animals were bred for several generations, so a possible accidental retinal phenotype is virtually excluded.

\subsubsection{Dark and light adaptation}

To absolutely exclude doubt on the dark adaptation of the animals before the scotopic measurements, preparatory procedures would need to be carried out under infrared light. Trials on using a night vision device turned out not practicable due to distorted distances towards the working desk, dizziness and blurring. This way, preparatory procedures on the animal, like electrode or thermometer placement could not be performed assuring minimal disturbance of the animal and exact placement of all recording devices. It needs to be considered, whether an infrared light system should get integrated into the dark working room to assure adequate preparation of the animal itself and the experimental settings

All preparatory procedures were performed in dim, red light emitted by red-coloured light bulbs (20W). Were these preparatory procedures performed in too much light? Were the animals still sufficiently dark adapted at the beginning of the scotopic measurements? A hint towards nonsufficient dark adaptation would be small amplitudes and non well-defined waveforms. Our ERGs showed well defined waveforms with reproducible amplitudes and latencies. We do not explain the small amplitudes of the second set of experiments with the NL2 animals by insufficient dark adaptation, as these animals were adapted in the same setting for the same time as all other experimental animals, who showed higher amplitudes. Maybe, when performing the preparatory procedures under infrared light conditions, absolute amplitudes would have been even higher. This problem can not be completely explained here. Comparing experiments under infrared light conditions would be needed for definite conclusions.

The photopic measurements were performed successively after the scotopic ERGs. Light adaptation was 10 minutes long before the photopic measurements. Still, this light adaptation was not performed from total dark adaptation, but after three runs of scotopic ERG measurements at different stimulus intensities. This may be one reason for our partly rather unsatisfying photopic responses. Still, photopic responses were clearly detectable, even showing differences between wild type and mutant mice. For the moment, no photopic ERGs are performed, but we need to 
consider, whether photopic measurements should be carried out one day after the scotopic measurements to assure light adaptation from total dark adaptation.

\subsubsection{Adaptation and exhaustion of the retina - Inter Stimulus Interval}

The purpose of the ISI is to maintain a state of dark adaptation between the flashes. Relatively short ISIs can be chosen for dim stimuli, which should be presented first and then, ISIs should lengthen with brighter flash intensities. A clear sign that the ISIs are inadequate is a steady decline in the amplitude of the dark-adapted ERG with increasing flash intensity (Peachey and Ball, 2003).

We considered, whether the retina may exhaust throughout our many measurements in a row. This could also be a reason for the saturation and decline of high-intensity response amplitudes. How long does it take the mutant retina to revert to the resting state compared to the WT? Were our ISIs long enough to give the presynaptic vesicle pool time to recover and recruit enough vesicles to the synaptic region to reach the maximum response possibly evoked by the next stimulus?

In our early experiments, we used ISIs of 2 seconds for all measurements at all stimulus intensities (see results of the $1^{\text {st }}$ set of experiments of the NL2 animals). The decline in amplitude described above is visible in the first experiments, whereas in later measurements (see later sets of NL2 and Protein 4.1 animals), which were performed with a 5 second ISI for stimulus lengths below $1 \mathrm{cds} / \mathrm{m}^{2}$ and 17 seconds for stimulus intensities beyond $1 \mathrm{cds} / \mathrm{m}^{2}$, this decline was not observed. However, these diverse results of the experiments with the NL2 animals may enable us to conclude on possible differential reaction of the retina to different ISIs.

\subsubsection{Correlation of oscillatory potentials to the ISI}

Using the short ISIs ( $1^{\text {st }}$ experimental set of NL2 animals), the OPs show a prominent decline in amplitude with increasing light intensity. Wachtmeister in 1973a stated that changes in amplitudes and energy content of the OPs will occur when recording the OPs under scotopic conditions. We observed clear OPs only in the scotopic measurements. As can be seen in the photopic results section, OPs could not be detected in our analysis.

The dominant frequency of the OPs decreases from about $150 \mathrm{~Hz}$ in a relatively more darkadapted condition to about $105 \mathrm{~Hz}$ in a more light adapted state as the ISI is shortened. The ener- 
gy of the oscillations is low when the ISI is long and increases when the ISI shortens. OPs of maximal energy and low frequency are recorded during mesopic conditions (Algvere and Wachtmeister 1972, Algvere et al. 1972, Wachtmeister 1973a and b, Wachtmeister and Hahn 1987). During mesopic conditions the retinal electrical response contains much rod activity. Thus, influence from the rod system to the inner layers of the retina affects the generation of the OPs.

Our observations concerning the OPs would be consistent with these statements. The maximum amplitudes of the OPs were reached at medium light intensities. All our OP frequencies lie very consistently between $100-120 \mathrm{~Hz}$.

Disruption of GABA-mediated pathways selectively and differentially reduces or abolishes the oscillations in the mudpuppy retina (Wachtmeister and Dowling, 1978). The earlier OPs (1-3) are more sensitive to the blocking of GABA-ergic pathways. Therefore, alterations of the earlier OPs should theoretically be able to indicate disturbances in the inhibitory feedback part of the retinal microcircuitry related to the ON-pathway of the retina and initiated by the amacrine cells. The later OPs are more vulnerable to the blocking of the glycine-sensitive network and so seem to be related to the OFF-effect in response to short flashes of light (Korol et al., 1975; Wachtmeister, 1980). OPs are also associated (Steinberg, 1966), maybe even directly correlated (Ogden, 1973) with rhythmic ganglion cell discharge. Membrane damage to the dendrites of the ganglion cells may affect the OPs (Vaegan and Millar, 1994), which may indicate a possible involvement of certain ganglion cells in the initiation of the activity in the inhibitory feedback pathway reflected by the OPs. Furthermore, the origin of the OPs is separate from that of the bwave (Ogden, 1973; Heynen et al., 1985). Muller cells are incapable of generating extracellular current flows with frequencies as high as those of the rapid oscillations (Ogden, 1973).

\subsubsection{Light application, light intensity}

Calibration of the stimulus source was performed by two different calibration systems as mentioned in the methods section. This calibration needs to be carried out with at least one calibrating system at least once a month to assure constant stimulus conditions. The LEDs, eventhough they are generally thought to have very long lifetimes, do get weaker by age and their light energy needs to be watched carefully. Pinto et al. observed partial failure of LEDs at least five times in four years of screening. Flashing stimuli were normal, but a steadily applied voltage did not 
produce a steady light from the LED. This gave the false impression that the cone ERG was much larger than it would have been had it been recorded properly.

At the very beginning of our ERG experiments, we did not use LEDs to produce a steady background illumination, but a regular flashlight. The flashlight was chosen so that the applied light intensity was approximately $30 \mathrm{cds} / \mathrm{m}^{2}$. Unfortunately, this flashlight tended to slack joints, which we soon realized, because the photopic ERG curves grew suddenly much larger and showed obvious oscillatory potentials. They looked more like the dark adapted ERGs we recorded and on checking, this was always due to failure of the background light. The photopic measurements we recorded did not show oscillatory potentials as clearly as the scotopic ERGs. The two types of waves were obviously distinguishable during the measurements.

\subsubsection{Moistening the electrode}

Sometimes, we observed heavy baseline drift in the ERG waves. Pinto et al. constituted that excess saline application to the cornea may cause distortion of the ERG as well as baseline shifts. The liquid junction potential is large and unstable, causes the baseline to shift and the waves to be displaced. Improper placement of the electrode or poor electrode-contact to the retina may also be reasons. In case of these disturbances, they suggest to keep the cornea rather dry, wet the electrode (in this case, a DTL fiber electrode was used) and replace the contact lens. These suggestions could not be transferred to our experiments, as we used a different electrode and no contact lens.

If a gel is used as conductant, this gel may dry out and stick to he cornea, which could cause damage to the cornea and inhibit signal conduction. If the animals are not measured a second time, using a carefully moistened conducting gel that is salt-free or at least containing isotonic salt concentration, is sufficient. But if the animals are supposed to be measured two or more times on different days, the electrode without protection may cause corneal damage and thus unreproducable responses in the following experiments. Further, by cutting the recording electrode from a furled spool, sharp edges may occur, which may also cause corneal damage. By embedding our silverchloride electrode into an agar-gel-block based on ringers solution, we did not have to use any gel or excess saline to keep the contact site between electrode and cornea moist. If more than one ERG recording is planned on the same animal at another time or day and no specialized contact lenses are available, we recommend to embed the electrode in a gel-like cover. 


\subsubsection{Different stimulus lengths}

Our experimental protocol for ERGs contains many runs with different stimulus lengths in a row, first under scotopic, then under photopic lighting conditions. Looking at the results, it may be worth to question, whether it is really necessary to run all three different stimulus lengths. The lowest light intensities with very short stimuli are definitely necessary if the threshold of the retinal response is questioned. The maximum stimulus intensities with longer stimuli are necessary to detect maximum capacity of the retinae and possible earlier tiring/saturation of the $\mathrm{KO}$ retinae in comparison to the wild types.

The medium stimulus lengths are questionable. There are certain components of the retinal response, that are best detected in mesopic lighting conditions (e.g. the OPs). But the ERG run with the $5 \mathrm{~ms}$ stimulus runs through the same light intensities tested in the $1 \mathrm{~ms}$ ERG run. This is the reason, why the ERG plots of the 1ms stimulus measurements were removed from the results section. The $1 \mathrm{~ms}$ runs did not provide us with significant additional information, that would change the conclusions on retinal function. Neither did they show significant changes in responses that were not visible in the $5 \mathrm{~ms}$ measurements.

It may be discussed, whether absolute values of the ERG components are of focused interest, or if the relation of responses between mutant and wild type animals is sufficient for a reasonable statement on retinal function when the experimental settings are always the same. On the other hand, responses with suspicious small amplitudes in both wildtype and knockout animals, may cloud statistically significant results.

Some of the discussed problems were not solved yet. Further ERG recordings and system calibrations will have to be carried out to eliminate failure sources. Since the experiments presented in this work were performed, the protocol for ERG measurements was reviewed. Our considerations mentioned were taken together and the protocol was modified. ERG measurements are performed with fewer recordings in a row. We reduced the number of test stimuli according to the protocol suggested by Roland Consult, not differentiating different stimulus lengths in the analysis. The ERG system will be and needs to be adapted dynamically to current standards continuously. This work describes a status quo and does not set a rigid framework for the experimental setting. It is supposed to give basic information and requirements to future experimenters who shall keep in mind that experimental settings are dynamic settings open to newer standards and changes according to the directed questions. 


\subsection{Neuroligin 2}

\subsubsection{NL2 is localized in the retina}

$\mathrm{GABA}_{\mathrm{A}}$ receptors are located in the dendrites of amacrine and ganglion cells and at bipolar cell axon terminals (Koulen et al., 1998a). Essrich et al. in 1998 reported that loss of $\mathrm{GABA}_{\mathrm{A}} \mathrm{R} \gamma 2$ subunits in mouse cortical neurons causes loss of synaptic GABAergic function.

Hoon et al. (2007b, Poster, and 2009) characterized the retinal morphology of NL2-deficient mouse retinae. NL2 was present in the OPL and IPL. Overall architecture of the two layers was normal. Ribbon synapse density and distribution in the OPL and IPL was also normal. In the wildtype retina, NL2 colocalized extensively with the $\mathrm{GABA}_{\mathrm{A}} \mathrm{R} \gamma 2$ subunit in the OPL and the IPL (next to subunits $\alpha 1, \alpha 2, \alpha 3$ ). In NL2-deficient retinae, the $\mathrm{GABA}_{\mathrm{A}} \mathrm{R} \gamma 2$ subunits were drastically reduced. Hoon et al. concluded, that NL2 deficiency in the end causes a decrease of functionally intact $\mathrm{GABA}_{\mathrm{A}}$ receptors.

Lui et al. in 2010 showed that NL2 was also localized presynapically within the ribbon synapseactive zone complex of the photoreceptor terminals. In their results, NL2 was not associated with inhibitory GABAergic synapses in the OPL. In the IPL, NL2 was present presynaptically in BC terminals connecting to amacrine cell processes.

The findings of Koulen et al. (1998a), Hoon et al. (2009) and Lui et al. (2010) taken together are consistent with the fact that NL2 is involved in postsynaptic GABAergic synapse differentiation (Varoqueaux et al., 2006). A decrease in functional $\mathrm{GABA}_{\mathrm{A}}$ receptors on amacrine cells due to NL2 deficiency in the bipolar cell axon terminal is plausible. NL2-deficieny may thus cause a functionally relevant impairment of amacrine cell activity (on BCs) in the IPL. The source of this impairment may already be found in synapse differentiation during synaptgenesis. 


\subsubsection{ERG components in NL2 mutant mice}

\subsubsection{Possible reasons for reduced ERG OP amplitudes in NL2 mutant retinae}

The OPs are sensitive to blocking of GABAergic pathways and disruption of these pathways diminishes them (Wachtmeister, 1980). Alterations of the OPs can indicate disturbances in the inhibitory feedback mechanisms in the retina initiated by the amacrine cells (Wachtmeister, 1998).

$\mathrm{GABA}_{\mathrm{C}}$ receptors are preferentially located in the IPL on BC axon terminals (Koulen et al., 1998a). Dark adapted ERG measurements from GABA $\mathrm{C}_{\mathrm{C}}$ deficient mice by McCall et al. in 2002 showed normal a- and b-waves, but increased number and amplitude of oscillatory potentials. They concluded that $\mathrm{GABA}_{\mathrm{C}}$ receptors may mediate local feedback inhibition to diminish transmission between bipolar cells and ganglion cells. Hull et al. in 2006 proposed that a $\mathrm{GABA}_{\mathrm{C}}$ receptor-mediated inhibitory current can provide a shunt to depolarizing potentials at the bipolar cell terminal. They suggested, that the involved $\mathrm{GABA}_{\mathrm{C}}$ receptors may act to limit glutamate release from $\mathrm{BC}$ terminals.

NL2 knockout mice showed slightly reduced $\mathrm{GABA}_{\mathrm{C}} \mathrm{R}$ diversity in the IPL (Hoon et al., 2009). Reduced $\mathrm{GABA}_{\mathrm{C}} \mathrm{R}$ activity may cause larger excitation at the rod bipolar cell, enhancing transmission to the following ganglion cells. The NL2 KO mice should (according to McCall et al., 2002 and Hull et al., 2006) show increased number and amplitude of OPs. However, in our experiments, they did not. In the second and third set of experiments, no statistically significant difference between NL2 WT and KO mice was observed at all, which could be explained by the only slightly yet not significantly reduced $\mathrm{GABA}_{\mathrm{C}} \mathrm{R}$ diversity. In the first set, using short ISIs, the NL2 KO mice showed statistically significant reduced amplitudes of OPs. None of our data sets shows the same results as McCall et al. in 2002.

One possible explanation leads away from the GABAergic system to the glycinergic system. Our findings of reduced OP amplitudes in the $1^{\text {st }}$ data set may correlate to the finding of increased expression of GlyT1 in NL2 deficient retinae by Hoon et al., 2009. GlyT1 is a glycine transporter which provides glycinergic amacrine cells with a high affinity uptake system for glycine (Pourcho and Goebel, 1985; Wassle et al., 1986). Glycine is thought to mediate vertical inhibition (Wassle, 2004). An OP amplitude reduction in NL2 deficient retinae could be the result of an increased inhibitory glycinergic effect on ON rod bipolar cells through the amacrine system. The increased levels of GlyT1 in order of deletion of NL2 might reflect some adaptive remodelling of the retinal network due to loss of NL2 and deficits in the GABAergic network. The deficit in the 
GABAergic pathway may be compensated by increased dendritic coverage by glycinergic amacrine cells (Hoon et al., 2009).

\subsubsection{Possible reasons for reduced ERG b-wave amplitudes in NL2 mutant retinae}

The scotopic b-wave response is mainly carried via ON rod bipolar cells. Rod bipolar cells are GABA-sensitive (Karschin and Wassle, 1990) and dominated by GABA $A_{C}$ receptors with slow dynamics (Eggers and Lukasiewicz, 2006; Frech and Backus, 2004). McCall et al. in 2002 found that $\mathrm{GABA}_{\mathrm{C}}$ knockout mice do not show any alterations of their ERG a- and b-wave amplitudes, but possible b-wave effects of NL2-deficiency and consequent GABA $R$ impairment shall be presented here.

Molnar and Werblin in 2007 reported that GABAergic amacrine cells inhibit (shape) the rod bipolar cell signal by acting on $\mathrm{GABA}_{\mathrm{C}}$ receptors. Hanitzsch et al. in 2004 showed that extracellular GABA added in high concentrations reduced the b-wave and the light response of the HCs. They concluded, that GABA is likely to cause suppression of $\mathrm{ON}$ rod BC responses to light stimuli. NL2 deficient mice revealed a compromised $\mathrm{GABA}_{C}$ receptor diversity in the IPL (Hoon et al., 2009). An important IPL-cell within the rod pathway is the A17 amacrine cell, which uses a $\mathrm{GABA}_{\mathrm{C}}$ receptor to feed back information from rod bipolar cells to rod bipolar cells. The A17 mechanisms are not yet clarified completely, but compromised $\mathrm{GABA}_{\mathrm{C}}$ receptor diversity in the IPL may affect the A17 cells and have an effect on the overall response of the rod bipolar cell circuit, affecting the b-wave of the ERG. The amacrine cells may not be able to confer inhibitory feedback properly onto the rod bipolars. In other words, the rod bipolar signal is not shaped in the way it should be, possibly resulting in impaired contrast detection. If this impairment is detectable by the ERG and reflected by an altered b-wave remains yet unclear, but possible.

Hoon et al. in 2009 observed compromised distribution of $\mathrm{GABA}_{\mathrm{C}}$ receptor labeling and elevated levels of GlyT1 (marker for glycinergic ACs) in the IPL. They also showed that the bipolar cell response to ganglion cells is shaped by inhibitory inputs to the bipolar cell mainly mediated by glycinergic amacrine cells. NL2-deficient retinae showed elevated levels of GlyT1 in the IPL, a marker for glycinergic amacrine cells. With possibly increased inhibitory amacrine cell activity on bipolar cells, reduced b-wave amplitudes could be promoted. Supporting data have been reported in 2009 by Mørkve and Hartveit, who showed evidence of presynaptic inhibitory glycinergic input from amacrine cells onto rod bipolar axon terminals. They stated, this inhibitory 
input is likely to suppress exocytosis from rod bipolar cells. Suppressed synaptic output could thus suppress the measurable electric activity.

Miura et al. in 2009 showed that intravitreal injection of APB (2-amino-4-phosphonobutyric acid), an agonist on metabotropic glutamate receptors (Slaughter and Miller, 1981), removed the bwave from the flash ERG. APB is known to block the synapses between the photoreceptors and ON-bipolar cells (inhibitory mGluR-mediated depolarizing response to light). Thus, in the NL2 KO mice, a malfunction of these inhibitory mGluR driven synaptic transmission may be an explanation for reduced $b$-wave amplitudes. This hypothesis does not correlate with the findings by Hoon et al., 2009, that the architecture of the OPL in the NL2 KO animals is normal with normal arborizations of bipolar cells, normal number of photoreceptors and normal number and spread of photoreceptor synapses. What might be considered is a dysfunction of synapses beyond normal architecture. 


\subsubsection{Possible reasons for reduced ERG a-wave amplitudes in NL2 mutant retinae}

The ERG a-wave is mainly associated to the photoreceptor current. The reduced a-waves of the NL-2 deficient animals may reflect some disurbance on the level of photoreceptor activity. The a-wave, as we remember, is mainly attributed to light-induced changes of the dark-current along the photoreceptor cell. The closure of CNG-channels in the PR outer segments is mediated by changes in intracellular cGMP-concentration via the phototransducion cascade.

Miura et al. in 2009 showed that intravitreal injection of the iGluR antagonist PDA (cis-2,3piperidine-dicarboxylic acid) into a mouse eye reduces a-wave amplitudes, removes the OPs but rarely affects the b-wave of the flash ERG. PDA is known to block transmission to hyperpolarizing $2^{\text {nd }}$ order neurons (OFF bipolar cells and and horizontal cells in the OPL) and all $3^{\text {rd }}$ order neurons (ganglion cells and amacrine cells in the IPL).

In the IPL, PDA blocks transmission of signals to horizontal cells. We wrote earlier that horizontal cells receive glutamatergic input via iGluRs and mediate lateral inhibition of photoreceptor responses. Changes in the iGluR-mediated pathways may change the PR current conditions and thus the a-wave of the ERG by influenzing the hyperpolarization/depolarization-level of the PR membranes.

\subsubsection{Recovery kinetics - the key to non-reproducible results?}

When taking a closer look at the retinal morphology of the NL2-deficient animals, why would the morphological changes only show functional sinificance with a short recovery time between the flashes? Within literature, not many works exist dealing with the temporal properties of retinal recovery, most of them based on computational models. Pepperberg et al. in 1996 provided a hypothesis on the recovery kinetics of the human rod phototransduction from a computational model on the "two-branched alpha-wave saturation function". Lyubarski and Pugh in 1996 also reported on the recovery phase of the rod photoresponse. For flash stimuli that produced $<3000$ activated rhodopsin molecules per rod, the effective lifetime of a single activated rhodopsin molecule was about $200 \mathrm{~ms}$. Recovery curves were isomorphic for these flash stimuli. The number of activated rhodopsin molecules was calculated by a formula taking into consideration several characteristics of the stimulus and the eye. Flash stimuli that produced $>3000$ activated rhodopsin molecules per rod evoked slightly different effects and the recovery curves were no longer proportional to stimulus intensity. They stated two hypotheses on prolonged rod recovery phases. Firstly, when a flash stimulus produces more than 3000 activated rhodopsin molecules per rod, 
about $0,03 \%$ of the rhodopsin molecules show effective lifetimes of $\sim 1$ sec. Secondly, 3000 activated rhodopsin molecules produce enough activated G-Proteins to exceed the total quantity of PDE $\gamma$ subunits necessary for hydrolysis of the G-Proteins. Excess activated G-Protein has to "wait" for inactivation by free PDE $\gamma$. Possibly, with shorter ISIs, more activated rhodopsin molecules are present and there is not enough time for G-Protein inactivation before the next flash. With ongoing stimuli, the amount of activated rhodopsin molecules may cumulate and, at the same time, fitting the saturated course of the ERG amplitudes, rhodopsin activation and GProtein inactivation processes reach maximum capacity. This could explain the plateau and decline of $\mathrm{a}$ - and b-wave amplitudes in the $1^{\text {st }}$ set of our experiments as well as the non-mantained significance using longer recovery phases between flashes.

It is difficult to conclude on possible affections of the recovery of the phototransduction cascade from the actual data. The short ISI and high stimuli may not have allowed the NL2-deficient retinae to recover sufficiently, which they can with longer ISIs. But which of the mentioned mechanisms underlies this finding in the special case of the NL2-KO animals yet remains unclear.

\subsubsection{Differing results in different data sets}

Discussing the results gained from our different experimental sets, it is very important not only to see the significant changes in the knockout mice, but just as important to reflect the reproduced non-significant results of the second and third data set. In the previus sections, we discussed possible mechanisms underlying a possible, but uncertain phenotype in the NL2 knockout mice. The observed morphological findings in the NL2 deficient retina were rather moderate. It is possible, bu also questionable, whether slight changes in number and distribution of single receptors or receptor subunits do really cause a functional impairment. Compensatory mechanisms in diverse inhibitory and/or excitatory circuits, which are not completely solved yet, may eviscerate the slight changes in the NL2-deficient retina.

Besides the visual function, the results of the auditory diagnostics did not show differences between NL2 knockout and wildtype mice. Although the auditory system functions differently from the retina, at least one very important structure exists in both systems - the ribbon synapses. These were not altered in the mutant retina. It may be assumed that the ribbon synapses of the cochlea of NL2-deficient mice are also unchanged, which would explain normal auditory function. No morphological studies of the cochlea have yet been performed in the NL2 knockout animals. 
In conclusion, all presented findings by ourselves and cited authors point out how very complex the mechanisms in the retina are. Our results do not entail definite statements, especially because our results were not reproduced in the different data sets. Still, they initiate different points of view and show that even small changes in the experimental setting influence the fine tuning and response of a system as complex as the retina. Stimulus processing in the retina does depend on temporal aspects of the stimulus as well as the inter-stimulus-intervals. Further studies may be directed to questions of response and recovery kinetics of the retina. 


\subsection{Protein 4.1}

\subsubsection{Protein 4.1 in the retina}

It is known that the cone photoreceptor contracts upon light-stimulation (so-called "cone retinomotor movements"). This contraction occurs almost exclusively in the so-called myoid (muscle-like) region of the cone inner segments and is mediated by actin (Burnside et al., 1982). Protein 4.1 modulates the interaction of $\alpha$-spectrin and actin filaments in cone inner segments. In 1991, Spencer et al. described a colocalization of protein 4.1 to $\alpha$-spectrin and f-actin in the myoid region of retinal cone photoreceptors. They accumulated and near the surface membrane of the myoid region of cone inner segments and were in proximity to a basket of f-actin filaments that extends from the inner segment to the external limiting membrane. Protein 4.1 and $\alpha$ spectrin may maintain cell morphology during contraction by providing membrane attachment sites for actin.

Protein 4.1 further inhibits motility and restricts distribution of integral membrane proteins like for example the $\mathrm{Na} / \mathrm{K}$-ATPase by binding to integral membrane proteins and the spectrin meshwork (Spencer et al., 1991).

Giebelhaus et al. in 1988 blocked normal expression of protein 4.1 through antisense protein 4.1 RNA and found, that showed that this perturbed the normal interdigitation of PR outer segments with the RPE. Under physiological conditions, retinal and membrane particles are processed in the PR outer segments to form the rhodopsin molecules with opsin. These data suggest that reduced expression of a single membrane skeleton protein like Protein 4.1 may perturb normal cellular interactions of the retina and further play a role in bidirectional transport mechanisms between the RPE and the photoreceptor outer segments.

Glutamate is the major excitatory neurotransmitter in the CNS. Glutamate receptors were distinguished into three different classes (AMPA, NMDA and kainate) by Hollmann and Heinemann in 1994. The synaptic localization, clustering and immobilization of neurotransmitter receptors and ion channels play important roles in synapse formation and synaptic transmission. The immobilization of these receptors is mediated via the actin cytoskeleton of the cell and depends on the integrity of the f-actin network (Allison et al., 1998; Kim and Lisman, 1999).

Shen et al. in 2000 suggested that 4.1G and 4.1N may cross-link an AMPA-receptor to the actin cytoskeleton in excitatory synapses. They showed that the AMPA receptor subunit GluR1 interacts with the actin-associated $4.1 \mathrm{G}$ and $4.1 \mathrm{~N}$. Protein $4.1 \mathrm{G}$ and $4.1 \mathrm{~N}$ bind to the $\mathrm{C}$-terminus of 
GluR1. Furthermore, a consensus region within the C-terminal domain of $4.1 \mathrm{G}$ and $4.1 \mathrm{~N}$ is sufficient to mediate the binding. Wozny et al. in 2009 instead showed that scaffolding proteins $4.1 \mathrm{G}$ and $4.1 \mathrm{~N}$ do not have a crucial role in glutamatergic synaptic transmission and the induction and maintenance of long-term plastic changes in synaptic efficacy. Their $4.1 \mathrm{G}$ and $4.1 \mathrm{~N}$ double-KO mice showed no changes in basic glutamatergic synaptic transmission.

\subsubsection{Protein 4.1 in synaptogenesis}

Studies in Xenopus by Spencer et al. in 1990 showed, that during retinal development, after the final mitoses, protein 4.1 is first associated with the inner plasma membrane of photoreceptors. After completion of retinal synaptogenesis, the distribution of protein 4.1 expands throughout the retina. Tsumoto et al. in 1988 discussed that in the developing visual cortex of the rat, protein 4.1 may be involved in regulating postsynaptic receptor function and synaptic plasticity in a calcium-dependent manner.

In 2001, Zhang and Benson showed in cultured hippocampal neurons, that f-actin is required for the development and maintenance of young synapses. In older synapses, maintenance of the synapse becomes independent of actin. Increased activity of the synapse can both reorganize and stabilize actin, indicating that actin may play a key role in synapse plasticity during development. Furthermore, they showed that both releasable and reserve pools of synaptic vesicles are initially tethered to f-actin during development, but that in older neurons they may be tethered to the cytoskeleton differently. In the older neuron, synaptic vesicle release grows less dependent on actin. Protein 4.1 is required for the formation of the membrane-stabilizing spectrin-actincomplex. Scott et al. in 2001 showed in forebrain postsynaptic density preparations, that 4.1 proteins have the potential to mediate the interactions of diverse components of postsynaptic densities. They showed, that the protein 4.1R C-terminal region bound PSD-spectrin and -actin. Given that the 4.1-spectrin interaction is regulated by the calcium-calmodulin-complex and also by phosphorylation processes, they concluded, 4.1 in the PSD may regulate the spectrin-actinlinkage in response to cellular signaling activity.

Hoon et al. (Poster, 2007a) described the retinal morphology of mutant mice lacking the protein 4.1 isoforms B, G and $\mathrm{N}$ by immunolabeling. There was no change in number or distribution of cone photoreceptor terminals in the TKO animals, but bassoon, a marker for all photoreceptor synapses, was statistically significantly changed $(\mathrm{p}<0,0001)$ in the 4.1 TKO retinae. Bassoondeficient mice show normal a-waves and significantly reduced b-waves (amplitude and frequen- 
cy) in the ERG (Dick, O. et al., 2003). This would be consistent with our results, as the 4.1 TKO animals showed significantly reduced b-wave amplitudes and in one row of measurements also significantly prolonged b-wave latencies. Zhang and Benson in 2001 stated that the decrease in synapse dependence on f-actin during development correlates well with the acquisition of preand postsynaptic scaffolding proteins like bassoon and PSD-95. The f-actin-4.1-complex may serve as a kind of placeholder during synaptogenesis. In protein 4.1-deficient mice, this placeholder should theoretically not be able to form properly.

Bassoon, as an important anchor around the ribbon, may take over the stabilizing functions of actin in the growing synapse. Initial synaptogenesis, dependent on actin, which is again dependent on diverse interactions of protein 4.1, could disrupted in the 4.1 TKO animals. This could be a possible explanation for the photoreceptor ribbons not being anchored to the presynaptic active zones. Impaired synaptic transmission from the PRs to BCs could explain reduced b-wave amplitudes in the 4.1 TKO animals.

\subsubsection{Protein 4.1 modulates glutamatergic retinal signaling}

Koulen et al. found in 1999 that the metabotropic glutamate receptor 8 (mGluR8 is) was expressed presynaptically in photoreceptor terminals. Further was mGluR8 located postsynaptically in HCs. At the IPL level, mGluR8 was located postsynaptically on dendrites of ACs and GCs. The mGluR8 on PR terminals was classified as an autoreceptor that evoked a decrease in intracellular calcium concentration as part of an inhibitory feedback loop playing its role in adjusting glutamate release from the PR terminal.

Rose et al. in 2008 found that the protein 4.1 isoforms B, G, and N showed comparable distribution to mGluR8 in the retina. Both were expressed in the OPL, the IPL and the GCL of the retina. Protein 4.1 bound to the C-terminus domain of mGluR8, promoted its cell surface localization and inhibited an mGluR8-mediated reduction of cAMP concentrations. They suggested several possible functional roles of protein 4.1 isoforms. These include diverse interactions with and modulation of the mGluR8 receptor.

The roles of protein 4.1 in amacrine, horizontal and ganglion cells are up to now putative. Some thoughts on this issue may nevertheless be named here. If glutamate so to speak modulates its own release via mGluR8 at the PR terminal, similar mechanisms may be assumed on other cells bearing mGluR8. 
Horizontal cells are depolarized by glutamate released from PR via postsynaptic iGluRs. If next to an iGluR, the mGluR8 is located postsynaptically in HCs, glutamate may thus have excitatory and inhibitory effects, respectively on the horizontal cells. Horizontal cells in turn contact PR ribbons to shape retinal signals in the means of center surround organization, also called "lateral inhibition". Horizontal cells cause depolarization of activated PRs on light stimulation. These two mechanisms together in parallel could again contribute to detection of contrasts and varying lighting conditions.

Calbindin in horizontal cells of 4.1 TKO mice was significantly altered (Hoon et al., 2007a, Poster). Wassle et al. in 1998 showed that calbindin is most likely not required for maintenance of the light-microscopic structure of the differentiated retina and suggested a functional rather than a morphological role for calbindin. If, as shown by Hoon et al. in 2009, 4.1 TKO animals do neither bear enough functional ribbons nor functionally normal horizontal cells, this could be a possible explanation for impaired signal modulation and possible impaired synaptic transmission at the OPL level.

In the previous sections we summarized multiple possible roles of protein 4.1 isoforms. How do all these findings correlate to the results of our experiments? In two sets of experiments, we showed significant reduction of all analysed ERG components in protein $4.1(\mathrm{~B} / \mathrm{G} / \mathrm{N})$ triple knockout mice.

The a-waves, as a correlate to the photoreceptor membrane current, were significantly smaller in the TKO mice. We earlier discussed possible reasons for reduced a-waves in the discussion of the NL2 mice. The a-wave is the measurable result of suppression of the PR dark current during a light flash. The more severe glutamate release is suppressed by light, the higher will the a-wave be. So, lower a-waves would correlate to a more sustained glutamate release after a light stimulus. How could the suppression of glutamate release be reduced?

Glutamate vesicles fuse with the presynaptic membrane in a calcium-dependent manner. As written before, glutamate regulates its own release via changes in intracellular calcium concentration via the mGluR8 autoreceptor. Some experimental data point out, that the mGluR8 receptor may structurally and functionally be dependent on interactions with protein 4.1 isoforms. Assumed, the mGluR8 receptors may be impaired either functionally or structurally by the deletion of protein 4.1 isoforms, the auto-feedback for the regulation (reduction) of glutamate release may be disturbed. This could result in more sustained glutamate release after a light flash in 4.1 TKOs 
compared to their WT littermate mice. A possible explanation for reduced a-wave amplitudes in our ERG experiments.

Secondly, the b-waves were also significantly reduced in the 4.1 TKO mice. The $b$-waves are mainly related to the activity of ON rod bipolar cells. It is known, that amacrine cells feedback onto rod bipolar cells. These feedback-synapses are thought to be GABAergic (Dong and Werblin, 1998). Hartveit described in 1999 that bipolar cell to amacrine cell signaling is mediated by iGluRs, whereas feedback from $A C s$ to $B C s$ is mediated by $\mathrm{GABA}_{\mathrm{A}}$ and $\mathrm{GABA}_{\mathrm{B}}$ receptors.

Last we found the amplitudes of the ERG oscillatory potentials in the $4.1 \mathrm{TKO}$ mice were significantly reduced. The OPs are mainly generated by complex inhibitory mechanisms related to the amacrine cells and GABAergic pathways.

Concrete explanations for all our ERG results in the 4.1 TKO mice cannot be made for the moment. Too little is yet known about the structural and functional roles of protein 4.1 isoforms in the different cell types of the retina. Nevertheless, we presented results that hint towards possible roles of proteins 4.1 and effects of protein 4.1 deficiency. Protein 4.1 isoforms do play a role in retinal development and physiology and deletion of 4.1 isoforms causes a reproducible visual phenotype. Herewith, we expanded the basis for future experimental questions concerning the structure and multiple functions of protein 4.1 isoforms. 


\section{Summary}

This work is concerned with retinal physiology and focused on results obtained from electroretinographic recordings. After presenting the method and obtained results, the results are discussed critically in the context of the literature. The manuscript is intended to provide important, however not all-encompassing information on retinal physiology and electroretinography to future experimenters on the subject. It constitutes an experimental setting, customized protocol and data analysis for ERG recordings in anaesthetized mice.

The measuring station, in which the experimental animals were tested, already existed and worked before starting the experiments for this work. Only few technical remodelings were necessary. We focused on the testing, modification and improvement of the stimulus protocols. They were altered several times. Furthermore, we programmed a reliable analysis routine which was connected to an overview illustration of the results. The raw data were analysed by a customized MATLAB routine, which was connected to a self-designed Excel Template. In this Excel Template, all results and necessary statistics are shown. The automated raw data analysis includes calculation of mean values, standard error of the means and t-tests. Preliminary illustrations of results are shown in diagrams.

We proved the systems' reliability for the characterization of retinal function and malfunction by measuring ERGs in different types of mutant mice with synaptic defects. In the discussion of the manuscript, we compare our experimental setting and protocols to ERG protocols of other scientific groups. Error susceptibility and differentially discussed ERG issues (e.g. adaptation to darkness, tiring of the retina) we were confronted with during development and application of the setup and recording protocols are discussed.

The retina comprises a complex synaptic network, in whose development the proper localization of synaptic components and targeted differentiation of synapses is crucial to the functioning of the whole system. The results presented and discussed in this manuscript were obtained from two different types of mutant mice with synaptic defects. The knocked-out proteins are involved in different steps of synaptogenesis and/or differentiation of synapses. In the discussion, results presented in the literature concerned with the affected proteins are merged with our own results. Mostly, the results complemented one another.

Differing results found after altering the measurement paradigms and stimulus protocol in the earlier stages of experiments were shown for the Neuroligin 2 knockout animals. Three different 
data sets were obtained from the Neurologin 2 mutant mice. The second set was published by Hoon et al. in 2009. All three sets of acquired data are shown in this work to illustrate how sensitive the retina is to changing experimental settings and to discuss possible technical reasons for the differing results. On the whole, dysfunction of GABAergic synapse differentiation and inhibitory signalling pathways in the retina may result from deletion of Neuroligin 2.

Reliability of the adjusted experimental system was proved by later experiments carried out on the Protein 4.1 triple knockout mice. The Protein 4.1 triple knockout mice gave reproducible results in two sets of experiments under almost identical stimulus conditions. The difference between the two sets of experiments was the number of presented stimuli. Fewer presented stimuli resulted in significantly higher absolute amplitudes of the ERG waves (same observation for the Neuroligin 2 animals in the second and third set of experiments). Protein 4.1 isoforms have been characterized extensively in their genetics and their interactions with cytoskeleton components. Functional data however are still rare and mostly speculative. Our suggestions for possible functional roles of protein 4.1, that derive from our results and comparison with existing literature, expand the basis for future experimental questions on the protein 4.1 isoforms.

Auditory testing was also performed in both types of mutant mice. The number of tested animals was very small and we did not obtain statistically significant changes in hearing thresholds between the wild type and mutant mice. The synaptic mutations carried by the tested animals mainly affect development and/or function of inhibitory signal transmission pathways the retina. Inhibition of signals plays a crucial role in retinal physiology. Possibly, auditory function in the tested animals is normal because in auditory physiology, inhibitory pathways do not play a comparably crucial role and are not affected significantly by the mutations. Future scientific questions could be targeted towards the issue of hearing function and cochlear morphology in the Neuroligin 2 and Protein 4.1 mutant mice.

Electroretinographic recordings are presently performed in our laboratory and will be carried out in the future. It is necessary to stay advertent and open towards criticism on our experimental setting. Regular calibration of the system is necessary to avoid systematic errors in results.

Overall we conclude, that our ERG setup, stimulus protocol, data analysis and data illustration function reliably and enable us to conclude scientifically and correctly on normal or impaired retinal function. 


\section{Appendix}

\subsection{References}

Algvere P, Wachtmeister L (1972): On the oscillatory potentials of the human electroretinogram in light and dark adaptation. II. Effect of adaptation to background light and subsequent recovery in the dark. A Fourier analysis. Acta Ophthalmol (Copenh) 무, 837-862

Algvere P, Wachtmeister L, Westbeck S (1972): On the oscillatory potentials of the human electroretinogram in light and dark adaptation. I. Thresholds and relation to stimulus intensity on adaptation to short flashes of light. A Fourier analysis. Acta Ophthalmol (Copenh) $\underline{50}$, 735-759

Allison DW, Gelfand VI, Spector I, Craig AM (1998): Role of actin in anchoring postsynaptic receptors in cultured hippocampal neurons: differential attachment of NMDA versus AMPA receptors. J Neurosci 18, 2423-2436

Ames JB, Levay K, Wingard JN, Lusin JD, Slepak VZ (2006): Structural Basis for Calciuminduced Inhibition of Rhodopsin Kinase by Recoverin. J Biol Chem 281, 37237-37245

Arden, GB, Brown KT (1965): Some Properties of Components of the Cat Electroretinogram Revealed by Local Recording under Oil. J Physiol 176, 429-461

Barraco R, Bellomonte L, Brai M, Anastasi M (2006): Analysis of the human a-wave ERG component. Physiol Meas 27, 881-899

Biederer T, Sudhof TC (2000): Mints as adaptors. Direct binding to neurexins and recruitment of munc18. J Biol Chem 275, 39803-39806

Biedermann F: Zentrale Verarbeitung akustischer Informationen nach unilateralen Läsionen im auditorischen Di- und Telencephalon. Nat. Diss. Leipzig 2004

Bolliger MF, Frei K, Winterhalter KH, Gloor SM (2001): Identification of a novel neuroligin in humans which binds to PSD-95 and has a widespread expression. Biochem $\mathrm{J} \underline{356}, 581-588$

Brandstatter JH, Fletcher EL, Garner CC, Gundelfinger ED, Wassle H (1999): Differential expression of the presynaptic cytomatrix protein bassoon among ribbon synapses in the mammalian retina. Eur J Neurosci 11, 3683-3693

Brindley GS, Hamasaki GI (1963): The properties and nature of the R membrane of the frog's eye. J Physiol 167, 599-606

Brown KT (1968): The eclectroretinogram: its components and their origins. Vision Res $\underline{8}$, 633677

Burnside B, Smith B, Nagata M, Porrello K (1982): Reactivation of contraction in detergentlysed teleost retinal cones. J Cell Biol 92, 199-206

Cobb W, Morton HB (1952): The human retinogram in response to high-intensity flashes. Electroencephalogr Clin Neurophysiol 4, 547-556

Cohen CM, Foley SF (1980): Spectrin-dependent and -independent association of F-actin with the erythrocyte membrane. J Cell Biol ㅌ6, 694-698

Connaughton V: Glutamate and glutamate receptors in the vertabrate retina. On www.webvision.med.utah.edu last updated May $7^{\text {th }} 2007$, downloaded on Dec $06^{\text {th }} 2011$

Dacheux RF, Raviola E (1986): The rod pathway in the rabbit retina: a depolarizing bipolar and amacrine cell. J Neurosci $\underline{6}, 331-345$ 
Deniz S, Wersinger E, Schwab Y, Mura C, Erdelyi F, Szabo G, Rendon A, Sahel JA, Picaud S, Roux M (2010): Mammalian retinal horizontal cells are unconventional GABAergic neurons. J Neurochem $\underline{116}, 350-362$

Dick E, Miller RF, Bloomfield S (1985): Extracellular K+ activity changes related to electroretinogram components. II. Rabbit (E-type) retinas. J Gen Physiol 85 911-931

Dick O, Hack I, Altrock WD, Garner CC, Gundelfinger ED, Brandstatter JH (2001): Localization of the presynaptic cytomatrix protein Piccolo at ribbon and conventional synapses in the rat retina: comparison with Bassoon. J Comp Neurol 439, 224-234

Dick O, tom Dieck S, Altrock WD, Ammermuller J, Weiler R, Garner CC, Gundelfinger ED, Brandstatter JH (2003): The presynaptic active zone protein bassoon is essential for photoreceptor ribbon synapse formation in the retina. Neuron $\underline{37}, 775-786$

Dong CJ, Werblin FS (1998): Temporal contrast enhancement via GABAC feedback at bipolar terminals in the tiger salamander retina. J Neurophysiol $\underline{79}, 2171-2180$

Eggers ED Lukasiewicz PD (2006): Receptor and transmitter release properties set the time course of retinal inhibition. J Neurosci $\underline{26}$, 9413-9425

Essrich C, Lorez M, Benson JA, Fritschy JM, Luscher B (1998): Postsynaptic clustering of major GABAA receptor subtypes requires the gamma 2 subunit and gephyrin. Nat Neurosci $\underline{1}$, 563-571

Feigenspan A, Bormann J (1994): Differential pharmacology of GABAA and GABAC receptors on rat retinal bipolar cells. Eur J Pharmacol 288, 97-104

Feo CJ, Fischer S, Piau JP, Grange MJ, Tchernia G (1980): 1st instance of the absence of an erythrocyte membrane protein (band 4(1)) in a case of familial elliptocytic anemia. Nouv Rev Fr Hematol 22, 315-325

Fletcher EL, Koulen P, Wassle H (1998): GABAA and GABAC receptors on mammalian rod bipolar cells. J Comp Neurol 396, 351-365

Foerster MH, van de Grind WA, Grusser OJ (1977): Frequency transfer properties of three distinct types of cat horizontal cells. Exp Brain Res 29, 347-366

Fowler V, Taylor DL (1980): Spectrin plus band 4.1 cross-link actin. Regulation by micromolar calcium. J Cell Biol $\underline{85}, 361-376$

Franze, K, Grosche J, Skatchkov SN, Schinkinger S, Foja C, Schild D, Uckermann O, Travis K, Reichenbach A, Guck J (2007): Muller cells are living optical fibers in the vertebrate retina. Proc Natl Acad Sci USA 104, 8287-8292

Frech MJ, Backus KH (2004): Characterization of inhibitory postsynaptic currents in rod bipolar cells of the mouse retina. Vis Neurosci 21, 645-652

Freed MA, Smith RG, Sterling P (2003): Timing of quantal release from the retinal bipolar terminal is regulated by a feedback circuit. Neuron $\underline{38}, 89-101$

Frishman, LJ: Origins of the electroretinogram; in: Principles and Practice of Clinical Electrophysiology of Vision; hrsg. v. Heckenlively JR, Arden GB, Nusinowitz S unter Mitarbeit namhafter Autoren; The MIT Press, Cambridge, MA 2006, 139-184

Giebelhaus DH, Eib DW, Moon RT (1988): Antisense RNA inhibits expression of membrane skeleton protein 4.1 during embryonic development of Xenopus. Cell $\underline{53}$, 601-615

Graf ER, Zhang X, Jin SX, Linhoff MW, Craig AM (2004): Neurexins induce differentiation of GABA and glutamate postsynaptic specializations via neuroligins. Cell $\underline{119}, 1013-1026$ 
Granit R (1933): The components of the retinal action potential in mammals and their relation to the discharge in the optic nerve. J Physiol 77, 207-239

Greengard P, Valtorta F, Czernik AJ, Benfenati F (1993): Synaptic vesicle phosphoproteins and regulation of synaptic function. Science 259 , 780-785

Grunert U (1999): Distribution of GABAA and glycine receptors in the mammalian retina. Clin Exp Pharmacol Physiol 26, 941-944

Gurevich EV, Gurevich VV (2006): Arrestins: Ubiquitious regulators of cellular signalling pathways. Genome Biol ㄱ, 236

Gurevich L, Slaughter MM (1993): Comparison of the waveforms of the ON bipolar neuron and the b-wave of the electroretinogram. Vision Res $\underline{33}, 2431-2435$

Hack I, Peichl L, Brandstatter JH (1999): An alternative pathway for rod signals in the rodent retina: rod photoreceptors, cone bipolar cells, and the localization of glutamate receptors. Proc Natl Acad Sci USA 96, 14130-14135

Hanitzsch R, Kuppers L, Flade A (2004): The effect of GABA and the GABA-uptake-blocker NO-711 on the b-wave of the ERG and the responses of horizontal cells to light. Graefe's Arch Clin Exp Ophthalmol 242, 784-791

Hartveit E (1999): Reciprocal synaptic interactions between rod bipolar cells and amacrine cells in the rat retina. J Neurophysiol $\underline{81}, 2923-2936$

Heynen H, Wachtmeister L, van Norren D (1985): Origin of the oscillatory potentials in the primate retina. Vision Res $\underline{25}, 1365-1373$

Hollmann M, Heinemann S (1994): Cloned glutamate receptors. Annu Rev Neurosci 17, 31 108

Holt M, Cooke A, Neef A, Lagnado L (2004): High mobility of vesicles supports continuous exocytosis at a ribbon synapse. Curr Biol $\underline{14}$ 173-183

Hood DC, Birch DG (1990): The A-wave of the human electroretinogram and rod receptor function. Invest Ophthalmol Vis Sci 31, 2070-2081

Hoon M, Bauer G, Moser T, Brose N, Varoqueaux F, Ivanovic A (2007a): Loss of 4.1 proteins leads to mislocalisation of synapse components in the mouse retina. Poster presented by Hoon M at the European Retina Meeting in Frankfurt/Main, Germany; October 4-6, 2007

Hoon M, Bauer G, Patrizi A, Sassoe-Pognetto M, Fritschy M, Moser T, Brose N, Falkenburger B, Varoqueaux F (2007b): Morphological and functional characterization of neuroligin 2 in the mouse retina. Poster presented by Hoon M at "Gephyrin: where do we stand, where do we go?" in Chexbres, Switzerland; September 9 - 11, 2007

Hoon M, Bauer G, Fritschy JM, Moser T, Falkenburger BH, Varoqueaux F (2009): Neuroligin 2 controls the maturation of GABAergic synapses and information processing in the retina. J Neurosci $\underline{29}$, 8039-8050

http://www.atlasophthalmology.com (downloaded on Dec. $20^{\text {th }}, 2010$ )

http://www.birket.com/technical-library/143/ (downloaded on April, 15 ${ }^{\text {th }}, 2011$ )

http://www.colorado.edu/intphys/Class/IPHY3430-200/008sensory.htm (downloaded on Dec 16th. 2010)

http://www.webvision.med.utah.edu/index.html (continuous checkup online)

http://webvision.med.utah.edu/sretina.html (downloaded Dec $6^{\text {th }}$ 2011)

Hull C, Li GL, von Gersdorff H (2006): GABA transporters regulate a standing GABAC receptor mediated current at a retinal presynaptic terminal. J Neurosci $\underline{26}, 6979.6984$ 
Ichtchenko K, Hata Y, Nguyen T, Ullrich B, Missler M, Moomaw C, Sudhof TC (1995): Neuroligin 1: a splice site-specific ligand for beta-neurexins. Cell $\underline{81}, 435-443$

Ichtchenko K, Nguyen T, Sudhof TC (1996): Structures, alternative splicing, and neurexin binding of multiple neuroligins. J Biol Chem 271, 2676-2682

Irie M, Hata Y, Takeuchi M, Ichtchenko K, Toyoda A, Hirao K, Takai Y, Rosahl TW, Sudhof TC (1997): Binding of neuroligins to PSD-95. Science 277, 1511-1515

Jaissle GB, May CA, Reinhard,J, Kohler K, Fauser S, Lutjen-Drecoll E, Zrenner E, Seeliger MW (2001): Evaluation of the rhodopsin knockout mouse as a model of pure cone function. Invest Ophthalmol Vis Sci $\underline{42}, 506-513$

Junqueira LC, Carneiro J: Basic histology, $11^{\text {th }}$ edition; McGraw-Hill Professional, New York 2005

Kandel ER, Schwartz JH, Jessell TM: Principles Of Neural Science. $4^{\text {th }}$ edition; The McGrawHill Companies, Inc, 2000

Karschin A, Wassle H (1990): Voltage- and transmitter-gated currents in isolated rod bipolar cells of rat retina. J Neurophysiol $\underline{63}, 860-876$

Kawamura S (1993): Recoverin has S-modulin activity in frog rods. J Biol Chem $\underline{268}$, 1457914582

Kelly GM, Zelus BD, Moon RT (1991): Identification of a calcium-dependent calmodulinbinding domain in Xenopus membrane skeleton protein 4.1. J Biol Chem 266, 12469-12473

Khimich D, Nouvian R, Pujol R, tom Dieck S, Egner A, Gundelfinger ED, Moser T (2005): Hair cell synatic ribbons are essential for synchronous auditory signalling. Nature $434,889-894$

Kim CH, Lisman JE (1999): A role of actin filament in synaptic transmission and long-term potentiation. J Neurosci $\underline{19}$, 4314-4324

Kolb, H: Functional Organization of the Retina; in: Principles and Practice of Clinical Electrophysiology of Vision; hrsg. v. Heckenlively JR, Arden GB, Nusinowitz S unter Mitarbeit namhafter Autoren; The MIT Press, Cambridge, MA 2006, 47-64

Komolov KE, Senin II, Kovaleva NA, Christoph MP, Churumova VA, Grigoriev II, Akh$\operatorname{tar}$ M, Philippov PP, Koch KW (2009): Mechanism of rhodopsin kinase regulation by recoverin. J Neurochem $\underline{110}, 72-79$

Korol S, Leuenberger PM, Englert U, Babel J (1975): In vivo effects of glycine on retinal ultrastructure and averaged electroretinogram. Brain Res 97, 235-251

Koulen P, Brandstatter JH, Enz R, Bormann J, Wassle H (1998a): Synaptic clustering of $\mathrm{GABA}(\mathrm{C})$ receptor rho-subunits in the rat retina. Eur J Neurosci $\underline{10}$, 115-127

Koulen P, Malitschek B, Kuhn R, Bettler B, Wassle H, Brandstatter JH (1998b): Presynaptic and postsynaptic localization of GABA(B) receptors in neurons of the rat retina. Eur J Neurosci $\underline{10}, 1446-1456$

Koulen P, Kuhn R, Wassle H, Brandstatter JH (1999): Modulation of the intracellular calcium concentration in photoreceptor terminals by a presynaptic metabotropic glutamate receptor. Proc Natl Acad Sci USA 96, 9901-9914

Lenzi D, von Gersdorff $\mathbf{H}$ (2001): Structure suggests function: the case for synaptic ribbons as exocytotic nanomachines. Bioessays $\underline{23}, 831-840$

Leskov IB, Klenchin VA, Handy JW, Whitlock GG, Govardovskii VI, Bownds MD, Lamb TD, Pugh EN Jr, Arshavsky VY (2000): The gain of rod phototransduction: reconciliation of biochemical and electrophysiological measurements. Neuron 27, 525-537 
Lui L, Levinson JN, Noël G, Handrigan GR, Richman JM, El-Husseini A, Moukhles H (2010): Synaptic localization of Neuroligin 2 in the Rodent Retina: Comparative Study with the Dystroglycan-containing Complex. J Neurosci Res $\underline{88}, 837-849$

Lukasiewicz PD, Eggers ED, Sagdullaev BT, McCall MA (2004): GABAC receptor-mediated inhibition in the retina. Vision Res $\underline{44}$, 3289-3296

Lyubarski AL, Pugh EN Jr (2006): Recovery phase of the murine rod photoresponse reconstructed from electroretinographic recordings. J Neurosci 16, 563-581

Magupalli VG, Schwarz K, Alpadi K, Natarajan S, Seigel GM, Schmitz F (2008): Multiple RIBEYE-RIBEYE interactions create a dynamic scaffold for the formation of synatic ribbons. $\mathbf{J}$ Neurosci $\underline{28}$, 7954-7967

Mandell JW, Townes-Anderson E, Czernik AJ, Cameron R, Greengard P, De Camilli P (1990): Synapsins in the vertebrate retina: absence from ribbon synapses and heterogeneous distribution among conventional synapses. Neuron $\underline{5}$ 19-33

Matthews G, Fuchs P (2010): The diverse roles of ribbon synapses in sensory neurotransmission. Nat Rev Neurosci $\underline{11}, 812-822$

McCall MA, Lukasiewicz PD, Gregg RG, Peachey NS (2002): Elimination of the rho1 subunit abolishes $\mathrm{GABA}(\mathrm{C})$ receptor expression and alters visual processing in the mouse retina. $\mathrm{J} \mathrm{Neu}-$ rosci $\underline{22}, 4163-4174$

Miller RF, Dowling JE (1970): Intracellular responses of the Muller (glial) cells of mudpuppy retina: their relation to b-wave of the electroretinogram. J Neurophysiol $\underline{33}$, 323-341

Miller SS, Steinberg RH (1977): Active transport of ions across frog retinal pigment epithelium. Exp Eye Res 25, 235-248

Miura G, Wang MH, Ivers KM, Frishman LJ (2009): Retinal pathway origins of the pattern ERG of the mouse. Exp Eye Res $\underline{89}, 49-62$

Molnar A, Werblin F (2007): Inhibitory feedback shapes bipolar cell responses in the rabbit retina. J Neurophysiol 98, 3423-3435

Mørkve SH, Hartveit E (2009): Properties of glycine receptors underlying synaptic currents in presynaptic axon terminals of rod bipolar cells in the rat retina. J Physiol 587, 3813-3830

Nelson R, Kolb H (1985): A17: a broad-field amacrine cell in the rod system of the cat retina. J Neurophysiol 54, 592-614

Oakley B $2^{\text {nd }}$, Green DG (1976): Correlation of light-induced changes in retinal extracellular potassium concentration with the c-wave of the electroretinogram. J Neurophysiol $\underline{39}, 1117-1133$

Ogden TE (1973): The oscillatory waves of the primate electroretinogram. Vision Res, $\underline{13}, 1059$ 1074

Pan MF, Massey SC (2007): Rod and cone input to horizontal cells in the rabbit retina. J Comp Neurol 500, 815-831

Parra M, Gascard P, Walensky LD, Snyder SH, Mohandas N, Conboy JG (1998): Cloning and characterization of $4.1 \mathrm{G}$ (EPB41L2), a new member of the skeletal protein 4.1 (EPB41) gene family. Genomics $\underline{49}$, 298-306

Parsons TD, Sterling P (2003): Synaptic ribbon. Conveyor belt or safety belt? Neuron $\underline{37}$, 379382

Pauli-Magnus D: Auditory Steady-State Responses (ASSR) und transiente auditorische Hirnstammpotenziale: Evaluation und Hörschwellenvergleich an Mausmodellen der sensorineuralen Schwerhörigkeit. Med. Diss. Göttingen 2010 
Peachey NS, Ball SL (2003): Electrophysiological analysis of visual function in mutant mice. Doc Ophthalmol 107, 13-36

Peichl L, González-Soriano J (1994): Morpholgical types of horizontal cell in rodent retinae: a comparison of rat, mouse, gerbil and guinea pig. Vis Neurosci 11, 501-517

Penn RD, Hagins WA (1969): Signal transmission along retinal rods and the origin of the electroretinographic a-wave. Nature 223, 201-204

Peters LL, Weier HU, Walensky LD, Snyder SH, Parra M, Mohandas N, Conboy JG (1998): Four paralogous protein 4.1 genes map to distinct chromosomes in mouse and human. Genomics $\underline{54}, 348-350$

Pepperberg DR, Birch DG, Hofmann KP, Hood DC (1996): Recovery kinetics of human rod phototransduction inferred from the two-branched alpha-wave saturation function. J Opt Soc Am A Opt Image Sci Vis $\underline{13}$, 586-600

Pieribone VA, Shupliakov O, Brodin L, Hilfiker-Rothenfluh S, Czernik AJ, Greengard P (1995): Distinct pools of synaptic vesicles in neurotransmitter release. Nature 375, 493-497

Pinto LH, Invergo B, Shimomura K, Takahashi JS, Troy JB (2007): Interpretation of the mouse electroretinogram. Doc Ophthalmol 115 127-136

Pourcho RG, Goebel DJ (1985): Immunocytochemical demonstration of glycine in retina. Brain Res $\underline{348}, 339-342$

Rao-Mirotznik R, Harkins AB, Buchsbaum G, Sterling P (1995): Mammalian rod terminal: architecture of a binary synapse. Neuron $\underline{14}$ 561-569

Rea R, Li J, Dharia A, Levitan ES, Sterling P, Kramer RH (2004): Streamlined synaptic vesicle cycle in cone photoreceptor terminals. Neuron $\underline{41}, 755-766$

Robson JG, Frishman LJ (1998-1999): Dissecting the dark-adapted electroretinogram. Doc Ophthalmol 95, 187-215

Robson JG, Saszik SM, Ahmed J, Frishman LJ (2003): Rod and cone contributions to the awave of the electroretinogram of the macaque. J Physiol 547, 509-530

Rose M, Dutting E, Enz R (2008): Band 4.1 proteins are expressed in the retina and interact with both isoforms of the metabotropic glutamate receptor type 8. J Neurochem $\underline{105}$, 2375-2387

Saszik SM, Robson JG, Frishman LJ (2002): The scotopic threshold response of the daradapted electroretinogram of the mouse. J. Physiol 15, 899-916

Scheiffele P, Fan J, Choih J, Fetter R, Serafini T (2000): Neuroligin expressed in nonneuronal cells triggers presynaptic development in contacting axons. Cell 101, 657-669

Schmitz F, Konigstorfer A, Sudhof TC (2000): RIBEYE, a component of synaptic ribbons: a protein's journey through evolution provides insight into synaptic ribbon function. Neuron $\underline{28}$, $857-872$

Scott C, Phillips GW, Baines AJ (2001): Properties of the C-terminal domain of 4.1 proteins. Eur J Biochem 268, 3709-3717

Serafini T (1999): Finding a partner in a crowd: neuronal diversity and synaptogenesis. Cell $\underline{98}$, 133-136

Shen L, Liang F, Walensky LD, Huganir RL (2000): Regulation of AMPA receptor GluR1 subunit surface expression by a 4. 1N-linked actin cytoskeletal association. J Neurosci 20, 79327940

Slaughter MM (1995): GABAb receptors in the vertebrate retina. Prog Ret Eye Res $\underline{14}$; 293-312 
Slaughter MM, Miller RF (1981): 2-amino-4-phosphonpbutyric acid: a new pharmacological tool for retina research. Science 211, 182-185

Smith WC: Phototransduction and Photoreceptor Physiology; in: Principles and Practice of Clinical Electrophysiology of Vision; hrsg. v. Heckenlively JR, Arden GB, Nusinowitz S unter Mitarbeit namhafter Autoren; The MIT Press, Cambridge, MA 2006, 35-78

Spencer M, Giebelhaus DH, Kelly GM, Bicknell J, Florio SK, Milam AH, Moon RT (1990): Membrane skeleton protein 4.1 in developing Xenopus: expression in postmitotic cells of the retina. Dev Biol 139, 279-291

Spencer M, Moon RT, Milam AH (1991): Membrane skeleton protein 4.1 in inner segments of retinal cones. Invest Ophthalmol Vis Sci $\underline{32}, 1-7$

Steinberg RH (1966): Oscillatory activity in the optic tract of cat and light adaptation. J Neurophysiol 29, 139-156

Steward O: Functional Neuroscience; hrsg. v. Steward O; Springer Verlag, New York, 2000

Tanaka T, Kadowaki K, Lazarides E, Sobue K (1991): $\mathrm{Ca}^{2+}$-dependent regulation of the spectrin/actin interaction by calmodulin and protein 4.1. J Biol Chem $\underline{266}, 1134-1140$

tom Dieck S, Brandstatter JH (2006): Ribbon synapses of the retina. Cell Tissue Res $\underline{326}$, 339346

tom Dieck S, Altrock WD, Kessels MM, Qualmann B, Regus H, Brauner D, Fejtova A, Bracko O, Gundelfinger ED, Brandstatter JH (2005): Molecular dissection of the photoreceptor ribbon synapse: physical interaction of Bassoon and RIBEYE is essential for the assembly of the ribbon complex. J Cell Biol $\underline{168}, 825-836$

Tsumoto T, Sato H, Sobue K (1988): Immunohistochemical localization of a membraneassociated, 4.1-like protein in the rat visual cortex during postnatal development. J Comp Neurol $\underline{271}, 30-43$

Ungewickell E, Bennett PM, Calvert R, Ohanian V, Gratzer WB (1979): In vitro formation of a complex between cytoskeletal proteins of the human erythrocyte. Nature $\underline{280}, 811-814$

Vaegan, Millar TJ (1994): Effect of kainic acid and NMDA on the pattern electroretinogram, the scotopic threshold response, the oscillatory potentials and the electroretinogram in the urethane anaesthetized cat. Vision Res $\underline{34}$ 1111-1125

Varoqueaux F, Aramuni G, Rawson RL, Mohrmann R, Missler M, Gottmann K, Zhang W, Sudhof TC, Brose N (2006): Neuroligins determine synapse maturation and function. Neuron $\underline{51}, 741-754$

Vinberg FJ, Strandman S, Koskelainen A (2009): Origin of the fast negative ERG component from isolated aspartate-treated mouse retina. J Vis $\underline{9}, 9$ 1-17

von Gersdorff H (2001): Synaptic ribbons: versatile signal transducers. Neuron $\underline{29}$, 7-10

Wachtmeister L (1973a): On the oscillatory potentials of the human electroretinogram in light and dark adaptation. 3. Thresholds and relation to stimulus intensity on adaptation to background light. Acta Ophthalmol (Copenh) 51, 95-113

Wachtmeister L (1973b): On the oscillatory potentials of the human electroretinogram in light and dark adaptation. IV. Effect of adaptation to short flashes of light. Time interval and intensity of conditioning flashes. A Fourier analysis. Acta Ophthalmol (Copenh) 1ㅗ, 250-269

Wachtmeister L (1980): Further studies of the chemical sensitivity of the oscillatory potentials of the electroretinogram (ERG) I. GABA- and glycine antagonists. Acta Ophthalmol (Copenh) $\underline{58}, 712-725$ 
Wachtmeister L (1998): Oscillatory potentials in the retina: what do they reveal. Prog Retin Eye Res $\underline{17}$, 485-521

Wachtmeister L, Dowling JE (1978): The oscillatory potentials of the mudpuppy retina. Invest Ophthalmol Vis Sci 17, 1176-1188

Wachtmeister L, Hahn I (1987): Spatial properties of the oscillatory potentials of the frog electroretinogram in relation to state of adaptation. Acta Ophthalmol (Copenh) $\underline{65}$, 724-730

Wassle H (2004): Parallel processing in the mammalian retina. Nat Rev Neurosci $\underline{5}$, 747-757

Wassle H, Schafer-Trenkler I, Voigt T (1986): Analysis of a glycinergic inhibitory pathway in the cat retina. J Neurosci $\underline{6}, 594-604$

Wassle H, Koulen P, Brandstatter JH, Fletcher EL, Becker CM (1998): Glycine and GABA receptors in the mammalian retina. Vision Res $\underline{38}, 1411-1430$

Wozny C, Breustedt J, Wolk F, Varoqueaux F, Boretius S, Zivkovic AR, Neeb A, Frahm J, Schmitz D, Brose N, Ivanovic A (2009): The function of glutamatergic synapses is not perturbed by severe knockdown of 4.1N and 4.1G expression. J Cell Sci $\underline{122}$, 735-744

Xu X, Karwoski CJ (1994a): Current source density (CSD) analysis of retinal field potentials. I. Methodological considerations and depth profiles. J Neurophysiol 2, 84-95

Xu X, Karwoski CJ (1994b): Current source density analysis of retinal field potentials. II. Pharmacological analysis of the b-wave and M-wave. J Neurophysiol $\underline{72}$, 96-105

Yamagata M, Sanes JR, Weiner JA (2003): Synaptic adhesion molecules. Curr Opin Cell Biol $\underline{15}, 621-632$

Yau KW, Hardie RW (2009): Phototransduction motifs and variations. Cell 139, 246-264

Young RW (1971): The renewal of rod and cone outer segments in the rhesus monkey. J Cell Biol $\underline{49}, 303-318$

Zhang W, Benson DL (2001): Stages of synapse development defined by dependence on Factin. J Neurosci 15, 5169-5181 


\subsection{Table of Illustrations and Tables}

Figure 1: Microscopic schematic of the retina

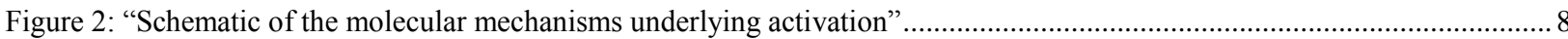

Figure 3: Schematic of iGluR- and mGlur-mediated synaptic transmission at the OPL level of the retina ..................................17

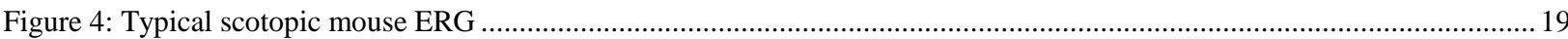

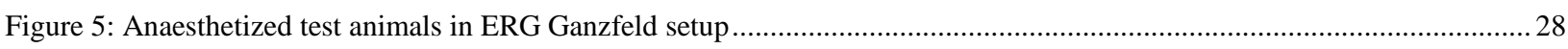

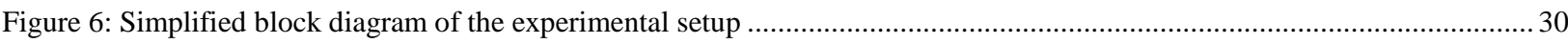

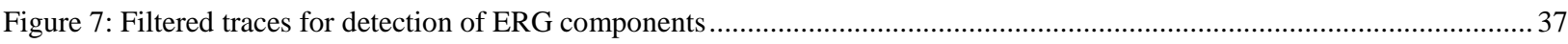

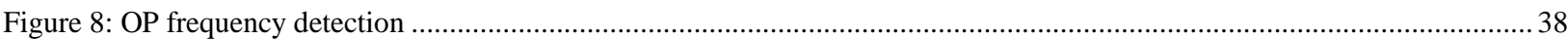

Figure 9: Overview illustration of data analysis for each animals' ERG measurement ............................................................39

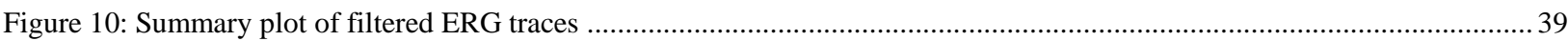

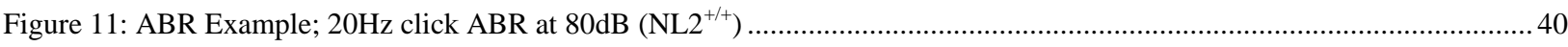

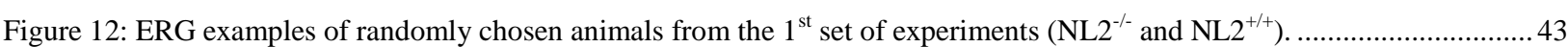

Figure 13: ERG examples of randomly chosen animals from the $2^{\text {nd }}$ set of experiments $\left(\mathrm{NL}^{-/-}\right.$and $\left.\mathrm{NL} 2^{+/+}\right)$..........................4

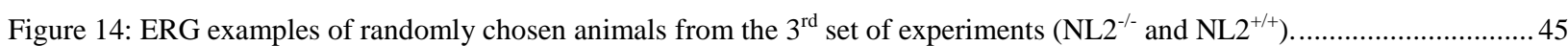

Figure 15: Amplitudes of a-waves, b-waves and OPs from the $1^{\text {st }}$ set of experiments ( $\mathrm{NL}^{-{ }^{-}}$and $\mathrm{NL} 2^{+/+}$).................................. 46

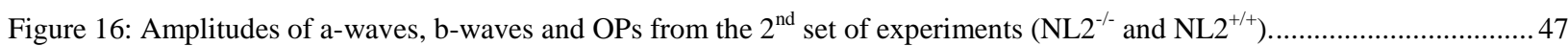

Figure 17: Amplitudes of a-waves, b-waves and OPs from the $3^{\text {rd }}$ set of experiments $\left(\mathrm{NL}^{-/-}\right.$and NL2 ${ }^{+/+}$) .............................48

Figure 18: Latencies of a-waves, b-waves and frequencies of OPs from the $1^{\text {st }}$ set of experiments (NL2 $2^{-/-}$and NL2 ${ }^{+/+}$)..............50

Figure 19: Latencies of a-waves, b-waves and frequencies of OPs from the $2^{\text {nd }}$ set of experiments $\left(\mathrm{NL} 2^{-/-}\right.$and NL2 $\left.{ }^{+/+}\right) \ldots \ldots \ldots \ldots . . .51$

Figure 20: Latencies of a-waves, b-waves and frequencies of OPs from the $3^{\text {rd }}$ set of experiments $\left(\mathrm{NL} 2^{-/-}\right.$and NL2 $\left.2^{+/+}\right) \ldots \ldots \ldots \ldots . . . .52$

Figure 21: Photopic b-wave and OP amplitudes and OP frequencies from $1^{\text {st }}$ set of experiments (NL2 ${ }^{-/-}$and NL2 ${ }^{+/+}$)................54

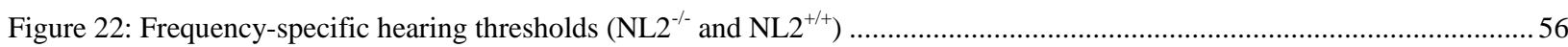

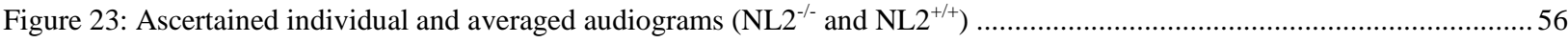

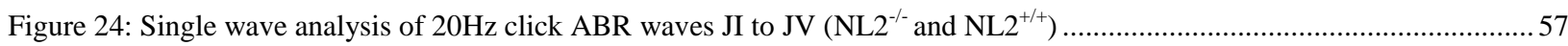

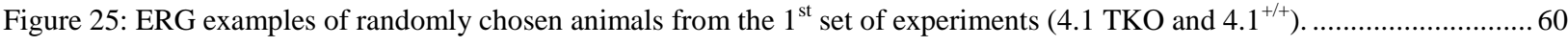

Figure 26: ERG examples of randomly chosen animals from the $2^{\text {nd }}$ set of experiments $\left(4.1\right.$ TKO and $\left.4.1^{+/+}\right)$........................61

Figure 27: Amplitudes of a-waves, b-waves and OPs from the $1^{\text {st }}$ set of experiments $\left(4.1\right.$ TKO and $\left.4.1^{+/+}\right) \ldots \ldots \ldots \ldots \ldots \ldots \ldots \ldots \ldots \ldots . . .63$

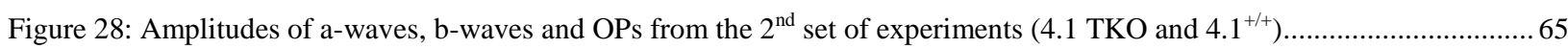

Figure 29: Latencies of a-waves, b-waves and frequencies of OPs from $1^{\text {st }}$ set of experiments $\left(4.1 \mathrm{TKO}\right.$ and $\left.4.1^{+/+}\right) \ldots \ldots \ldots \ldots \ldots \ldots .67$

Figure 30: Latencies of a-waves, b-waves and frequencies of OPs from $2^{\text {nd }}$ set of experiments $\left(4.1 \mathrm{TKO}\right.$ and $\left.4.1^{+/+}\right) \ldots \ldots \ldots \ldots \ldots . .68$

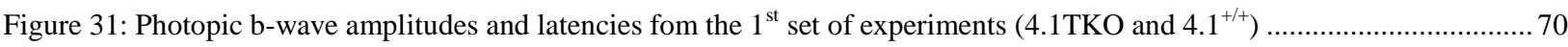

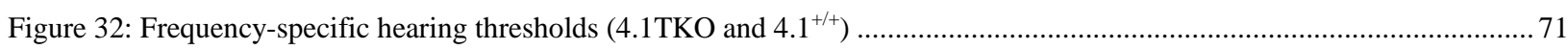

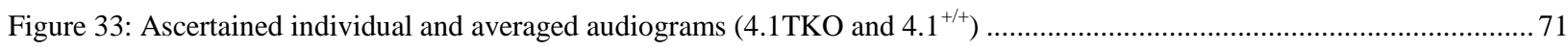

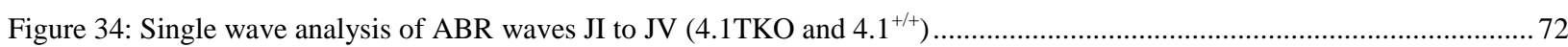


Table 1: Attenuation steps and resulting light intensities $\left(\right.$ in $\left.\mathrm{cds} / \mathrm{m}^{2}\right)$ at all used stimulus lengths $(0,1,1$ and $5 \mathrm{~ms})$....................32

Table 2: Flash intensity program of the Roland Consult Ganzfeld Q450 System for ERG experiments ......................................35

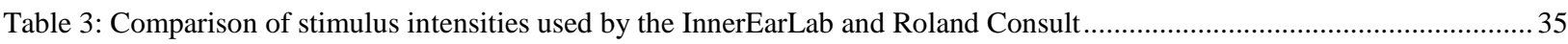

Table 4: Selection of different scotopic ERG protocols by different study groups ............................................................... 75 


\section{Acknowledgements}

Prof. Dr. med. Tobias Moser for granting this thesis, supervising the project, steady support of the author not only concerning this project and thesis and encouraging all involved lab members and cooperating scientists.

Dipl. Phys. Gerhard Hoch for excellent technical support, without who's patience and solid soft- and hardware knowledge the realization of electroretingraphic recordings, data analyses and creation of diagrams would not have been possible.

Christian Rüdiger for instructing the author on animal care issues, support in many series of measurements and critical discussions on technical issues and error sources during measurements. Furthermore, many thanks for kindly letting the author show his later on measured data of the NL2- and Protein 4.1 animals for this work.

Mrinalini (Tina) Hoon, Ph.D. for support during measurements, excellent cooperation on the tested animals and cooperation on the paper Hoon et al., 2009 "Neuroligin 2 controls the maturation of GABAergic synapses and information processing in the retina." J Neurosci 29 , 80398050

Dr. Frédérique Varoqueaux and Dr. phil. nat. Aleksandra Ivanovic from the Max-PlanckInstitute for Experimental Medicine for cooperation and providing the test animals and morphological data of the tested retinae. Further, Dr. Ivanovic for critically reading the final version of this thesis and constructive suggestions for improvement.

Dr. sc. agr. Christian Arglebe for critically reading an earlier version of this thesis and providing constructive corrections. 